Purdue University Purdue e-Pubs

ECE Technical Reports

Electrical and Computer Engineering

9-1-1992

\title{
Comparative Study of Motion Control Methods for a Nonlinear System
}

Daniel J. Pack,

Purdue University School of Electrical Engineering

Min Meng

Purdue University School of Electrical Engineering

A. C. Kak

Purdue University School of Electrical Engineering

Follow this and additional works at: http://docs.lib.purdue.edu/ecetr

Pack,, Daniel J.; Meng, Min; and Kak, A. C., "Comparative Study of Motion Control Methods for a Nonlinear System" (1992). ECE Technical Reports. Paper 273.

http://docs.lib.purdue.edu/ecetr/273

This document has been made available through Purdue e-Pubs, a service of the Purdue University Libraries. Please contact epubs@purdue.edu for additional information. 


\title{
COMPARATIVE STUdy OF Motion CONTROL METHODS FOR A NONLINEAR SYSTEM
}

\author{
DANIEL Jo PACK \\ MIN MENG \\ AVI C. KAK
}

TR-EE 92-41

SEPTEMBER 1992

SCHOOL OF EleCtRICAL ENGINEERING 


\title{
Comparative Study of Motion Control Methods for a Nonlinear System
}

\author{
Daniel J. Pack, Min Meng and A. C. Kak*
}

\begin{abstract}
What led to the novel ideas, some of them theoretical, presented in this paper is our highly experimental approach to nonlinear control. We actually designed and fabricated a two-link inverted pendulum, and learned about the relationships and the tradeoffs that exist in such a device. In the context of controlling an inverted pendulum, the novel ideas presented in this paper relate to i) the design of a fuzzy logic controller; ii) the design of a neuralnetwork controller; and iii) experimental implementation of a nonlinear controller based on differential-geometric notions; etc. etc. These novel ideas are presented in a comparative setting.

The comparative study presented compares, experimentally and by simulation, five different control schemes. We compare a PD controller, a linear quadratic controller, a nonlinear controller, a neural network controller and a fuzzy logic controller. We show how the controllable portion of the four dimensional space of control variables changes with the different control schemes. We do this by defining an effectiveness coefficient that is the ratio of the portion of the size of the controllable region to the size of the overall space as spanned by the position variables. Our overall conclusion may be summarized by the following values of the effectiveness coefficient for the five different control schemes:

$\begin{array}{lcc} & \text { Simulation } & \text { Experiment } \\ \text { PD Controller: } & 0.324 & 0.022 \\ \text { Linear Quadratic Controller: } & 0.524 & 0.234 \\ \text { Neural Network Controller: } & 0.785 & 0.272 \\ \text { Non-linear Controller: } & 0.248 & 0.203 \\ \text { Fuzzy Logic Controller: } & 0.349 & 0.262\end{array}$

It is interesting to note that while the superiority of the neural network controller is borne out by both the simulations and the experiments, the former overemphasizes the advantage by a wide margin. Also the simulation study would have us believe that a linear quadratic controller should be our second best choice, a conclusion not supported by actual experiments. The differences between the experiments and the simulations can be attributed to the difficulty of capturing in a simulation phenomena such as friction, motor dynamics, etc.
\end{abstract}

'Robot Vision Lab, 1285 EE Building, Purdue University, West Lafayette, IN 47907-1285 


\section{Table of Contents}

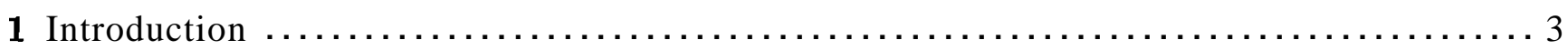

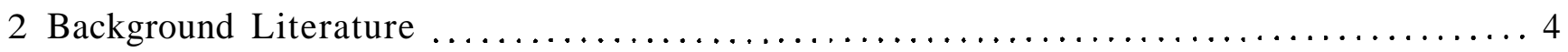

3 Experimental Setup and the Dynamics of the Nonlinear System ................. 5

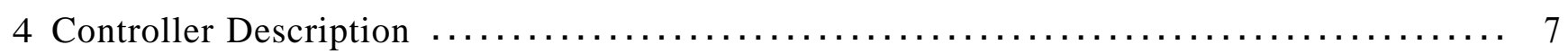

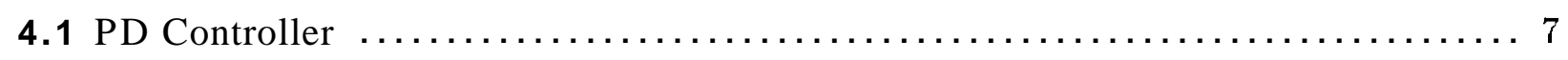

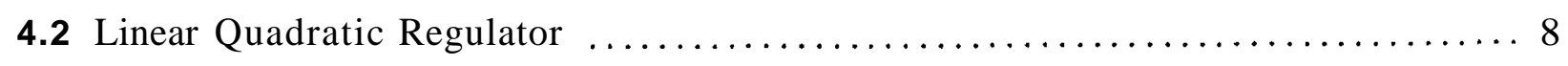

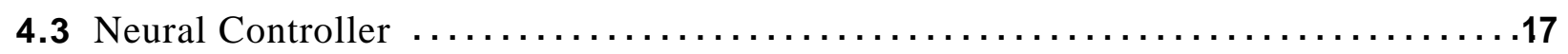

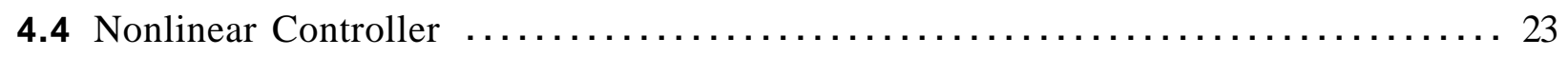

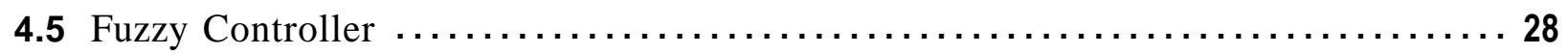

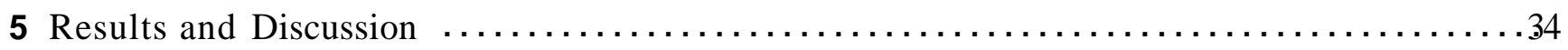

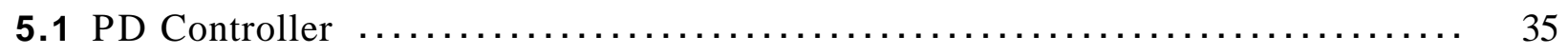

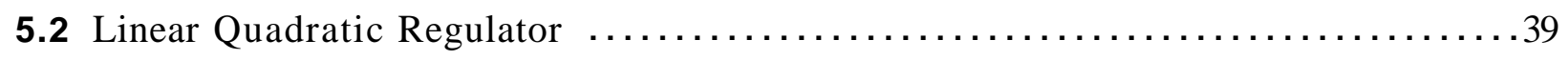

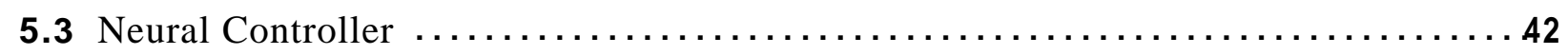

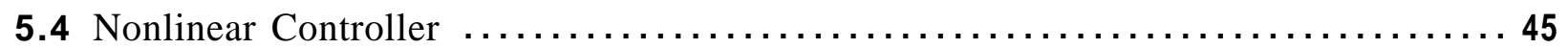

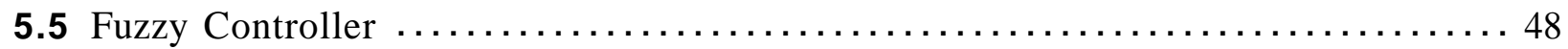

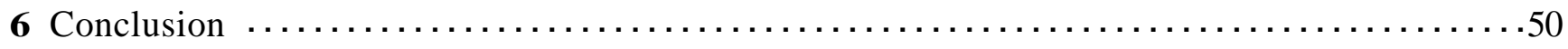

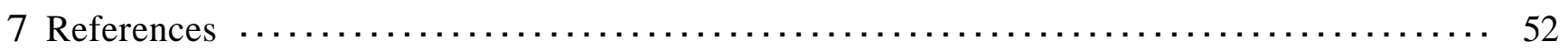

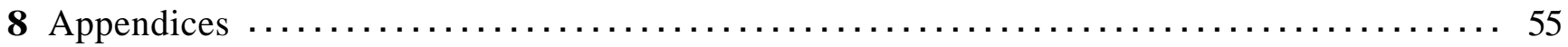


pack/meng/kak

\section{Comparative Study of Motion Control Methods for a Nonlinear System}

\section{Introduction}

Strictly speaking, the motion of a robot system involves nonlinear terms that describe inertia: gravity, and other effects. In the past, these terms have been ignored or simplified, often at the cost of high speed manipulability, because they implied an excessive computational burden for real-time control. However, with the advent of new hardware and software, we believe that it will be possible to accommodate the nonlinear constraints in the control schemes of the future. Moreover, there has come into existence a new class of nonlinear robotic control problems, such as those dealing with legged locomotion: that are not amenable to linearized implementations.

The last few years have seen the emergence of new methods for the control of nonlinear systems. The most prominent of these are the neural network based methods and the fuzzy logic based methods. Their proponents claim that these new methods are computationally superior to whatever approximations one may make to nonlinear control in order to make it computationally feasible. We may therefore say that these two new methods, the neural network based and the fuzzy logic based, and the traditional approaches, such as the linear and nonlinear approximations, constitute a body of competing methods for the control of a nonlinear system.

Given the competing approaches for nonlinear control, it is not clear today which approach should be selected for a given application. To remedy this deficiency in our knowledge, we have taken a small step in this paper and performed a comparative study on what has become the fruit fly of nonlinear control - the inverted pendulum problem. We have devised two criteria for comparing the different control schemes. The first of these, called the effectiveness coefficient, measures the ratio of the sizes of the actual to the ideal controllable regions of the space spanned by designated control variables. The second, the utilization coefficient, measures the economy in the total control input required to make a system transition from a designated initial state to the goal state.

With respect to above two criteria, the different control schemes will be compared first by computer simulation and then on an actual experimental setup. Since the peculiarities of a particular hardware can be their own sources of nonlinearities - nonlinearities that in most cases cannot be modeled theoretically - it is useful to compare the different schemes by computer simulation. Having said that, it goes without saying that a comparative study without actual experiments would be an empty exercise indeed. As we will show, the experiments reveal interesting departures from the performances predicted by simulations.

The paper is organized as follows. First, in Section 2, we discuss the related literature. In order to make explicit the nonlinearities in the particular inverted pendulum experiment that our results are based on, in Section 3 we will then discuss the experimental setup and its various parameters. Section 4 will then describe the different controllers that are the subject of comparison here. 
Simulations and experimental results will be presented in Section 5, and the results summarized in Section 6. The reader is referred to [24] for a more detailed version of this paper, which also contains an appendix on the configuration of the electronic hardware used for controlling the motor and for interfacing with the computer.

\section{Background Literature}

We will now briefly survey the literature on the emerging nonlinear methods for motion control. We will first mention some of the more prominent contributions that discuss the use of neural networks for nonlinear motion control. This will be followed by a mention of the recent work in nonlinear control based on differential geometry. Lastly, we will review the literature in the use of fuzzy logic for nonlinear motion control.

Showing just simulations, Barto et al. and .Anderson $[2,1]$ have demonstrated the use of neural networks to balance a pole that is hinged on a moving cart. In another simulation study, Kuschewski et al. have applied feedforward neural networks to learn the forward and the inverse dynamics of a single-link inverted pendulum controlled by a DC motor with a gear train [15]. In yet another simulation-based study, Helferty et al. [12] have reported on a neural network learning strategy for the control of a one-legged hopping machine. Among the contributions where researchers have reported on actual implementations and experiments, Miyamoto [21] has used a neural network to learn the inverse dynamics of a robot to control the robot trajectory. Sekiguchi et al. [32] have successfully demonstrated that it is possible to control a two-link inverted pendulum with a neural network controller. Nagata et al. [22] and, recently, Min and Kak [20] have used neural networks for robot navigation.

In the area of nonlinear control by using differential geometric concepts, many formalisms have been presented by scientists $[5,14,34]$. Starting early 80 's, theoretical extensions to the differential geometric context, the context that pertains to robotics, have begun to appear in the literature. Brockett [6] has showed how robot kinematics can be represented using Lie algebra and group theory. Again using differential geometric concepts, Paden and Sastry [25] have proved that a manipulator with six revolute joints contains an optimal reachability region if it is an elbow manipulator. Salam and Yoon [31] considered the exponential matrix representation of changes of rotations for the Stanford manipulator. Tarn et al. [35] have shown the necessary and sufficient conditions for the existence of a nonlinear feedback control for a general nonlinear system if we wish for it to be externally linearized using the differential geometric system theory. Applications of these notions to the control of robot motion are reported in $[10,26]$.

Based on the belief that human decisions are made on the basis of imprecise, non-numerical information, Zadeh [40] introduced the fuzzy set theory and fuzzy logic in mid 60's. Inspired by the successes of fuzzy logic in controlling nonlinear systems such as a steam engine [19], during the last few years many workers have attempted to apply this approach to a. wide range of control problems, including the problem of nonlinear motion control. Yamakawa [39] has recently 


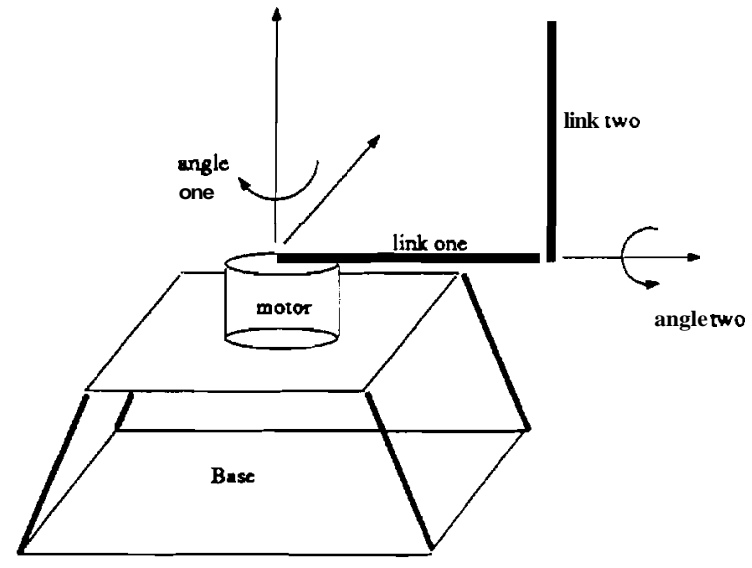

(a) schematic

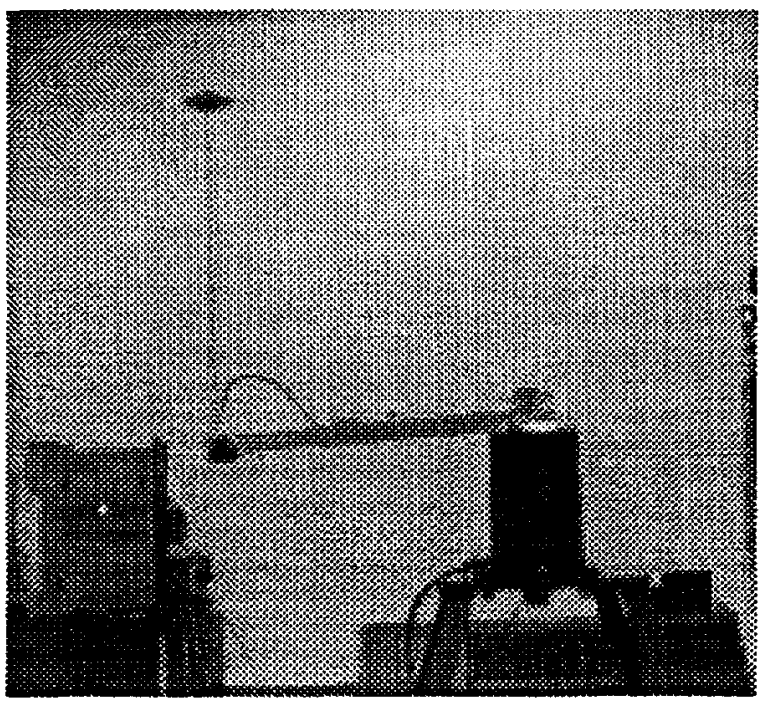

(b) experimental set-up

Figure 1: The inverted pendulum

demonstrated experimentally a fuzzy logic controller for the pole balancing problem. A fuzzy logic controller has also been advanced recently for helicopter control [38]. There still remain many unanswered questions to be addressed regarding such controllers, such as the lack of systematic procedures on how to select appropriate fuzzy membership functions and how to construct effective rules for a particular system.

\section{Experimental Setup and the Dynamics of the Nonlin- ear System}

We will now present the basic equations that describe the dynamics of a two-link inverted pendulum, which may be considered to be a single input and two output device. The first link, attached to a motor as shown in Fig. 1, rotates in a horizontal plane. The second link is attached to the end of the first link in such a manner that the plane of rotation of the second link will always be vertical and perpendicular to the plane of the rotation of the first link. The only input to the system is the torque to the motor to which the first link is attached. (If there were control inputs for both links, then the pendulum would become a two input, two output system - a much easier problem from a control standpoint.) For the motor, a direct drive servo actuator Dynaserv is used, and Transputers, high performance microprocessors that support parallel processing through onchip hardware, are used as the main computer. Note that the task of the controller is not only to balance the second link upright but to also position the first link at a prespecified angle. 
The dynamic equations are derived using the well known Euler-Lagrange equation

$$
\frac{d}{d t}\left[\frac{\partial L(\theta, \dot{\theta})}{\partial \dot{\theta}_{i}}\right]-\frac{\partial L(\theta, \dot{\theta})}{\partial \theta_{i}}=\tau
$$

$\mathrm{L}$ is the Largrange function $\mathrm{L}=K-P$, where $K$ and $P$ are the kinetic and potential energy of the system, respectively. Computing the appropriate parameter values for the system and substituting them into Eq. (1) yields the following dynamic equation

$$
\mathrm{D} \ddot{\theta}+\mathrm{C}(\theta, \dot{\theta})+\mathrm{G}(\theta)=\tau
$$

where

$$
\begin{gathered}
\mathrm{D}=\left[\begin{array}{cc}
\frac{1}{4} m_{1} l_{1}^{2}+m_{2} l_{1}^{2}+\frac{1}{4} m_{2} l_{2}{ }^{2} \sin ^{2} \theta_{2} & \frac{1}{2} m_{2} l_{1} l_{2} \cos \theta_{2} \\
\frac{1}{2} m_{2} l_{1} l_{2} \cos \theta_{2} & \frac{1}{4} m_{2} l_{2}{ }^{2}
\end{array}\right] \\
\mathrm{C}(\theta, \dot{\theta})=\left[\begin{array}{c}
\dot{\theta}^{T} \mathrm{C}_{1} \dot{\theta} \\
\dot{\theta}^{T} \mathrm{C}_{2} \dot{\theta}
\end{array}\right]
\end{gathered}
$$

and

$$
\begin{gathered}
\mathrm{C}_{1}=\left[\begin{array}{cc}
0 & \frac{1}{4} m_{2} l_{2}^{2} \sin \theta_{2} \cos \theta_{2} \\
\frac{1}{4} m_{2} l_{2}^{2} \sin \theta_{2} \cos \theta_{2} & -\frac{1}{2} m_{2} l_{1} l_{2} \sin \theta_{2}
\end{array}\right] \\
\mathrm{C}_{2}=\left[\begin{array}{cc}
-\frac{1}{4} m_{2} l_{2}^{2} \sin \theta_{2} \cos \theta_{2} & 0 \\
0 & 0
\end{array}\right] \\
\mathrm{G}(\theta)=\left[\begin{array}{c}
0 \\
-\frac{1}{2} m_{2} l_{2} g \sin \theta_{2}
\end{array}\right] \\
\tau=\left[\begin{array}{c}
\tau \\
0
\end{array}\right] .
\end{gathered}
$$

D is a symmetric non-singular inertia matrix where the diagonal component $D_{i i}$ along with its corresponding angular acceleration describes the acceleration force being exerted on the link $i$, and the off-diagonal term $D_{i j}$ represents the coupled acceleration force exerted on the link $i$. $\mathrm{C}$ consists of centripetal and coriolis terms where the diagonal terms $C_{i i}$ 's of $\mathrm{C}_{1}$ represent the centripetal forces generated by velocities of the link $i$ exerted on the link 1 while the off-diagonal terms $C_{i j}$ 's of $C_{1}$ describe the coriolis forces generated by velocities of the link $i$ and the link $j$ exerted on the link 1. Similar statement can be made for $C_{2}$. G depicts the gravitational term and the vector $\tau$ indicates the external torque applied to the system. For digital control of the system, it is convenient to change the Eq (2) into a form $\mathrm{x}=f(x, \dot{x}, \tau)$ where $\tau$ is the control input. Since the D matrix is always non-singular, its inverse exists and the transformation can be easily accomplished. And by denoting $x_{1}=\theta_{1}, x_{2}=\theta_{2}, x_{3}=\dot{\theta}_{1}$, and $x_{4}=\theta_{2}$, the dynamic equations for the system transform into a set of following first order nonlinear differential equations:

$$
\left[\begin{array}{c}
\dot{x_{1}} \\
\dot{x_{2}} \\
\dot{x_{3}} \\
\dot{x_{4}}
\end{array}\right]=\left[\begin{array}{c}
x_{3} \\
x_{4} \\
S_{1} \\
S_{2}
\end{array}\right]
$$


where

$$
\begin{aligned}
S_{1} & =\frac{m_{2}^{2} l_{2}^{2} \sin x_{2}}{8 \mathrm{I}} *\left(l_{2}^{2} x_{3} x_{4} \cos x_{2}-l_{1} l_{2} x_{4}^{2}+l_{1} l_{2} x_{4}^{2} \cos ^{2} x_{2}+2 l_{1} g \cos x_{2}-\frac{2}{m_{2} \sin x_{2}} \tau\right) \\
S_{2} & =\frac{m_{2} l_{1} l_{2} \sin x_{2}}{16 \mathrm{I}} *\left(4 m_{2} l_{2}^{2} x_{3} x_{4} \cos ^{2} x_{2}-4 m_{2} l_{1} l_{2} x_{4}^{2} \cos x_{2}+m_{1} l_{1} l_{2} x_{3}^{2} \cos x_{2}+2 m_{1} l_{1} g\right. \\
& \left.+8 m_{2} l_{1} g+4 m_{2} l_{1} l_{2} x_{3}^{2} \cos x_{2}+\frac{m_{2} l_{2}^{3} \sin ^{2} x_{2} \cos ^{2} x_{3}}{l_{1}}+\frac{2 m_{2} l_{2}^{2} g \sin ^{2} x_{2}}{l_{1}}-8 \tau \cot x_{2}\right)
\end{aligned}
$$

and

$$
I=\left(\frac{m_{1} m_{2}}{16}+\frac{m_{2}^{2}}{4}\right) l_{1}^{2} l_{2}^{2}+\frac{m_{2}^{2} l_{2}^{4} \sin ^{2} x_{2}}{16}-\frac{m_{2}^{2} l_{1}^{2} l_{2}^{2} \cos ^{2} x_{2}}{4}
$$

\section{Controller Descriptions}

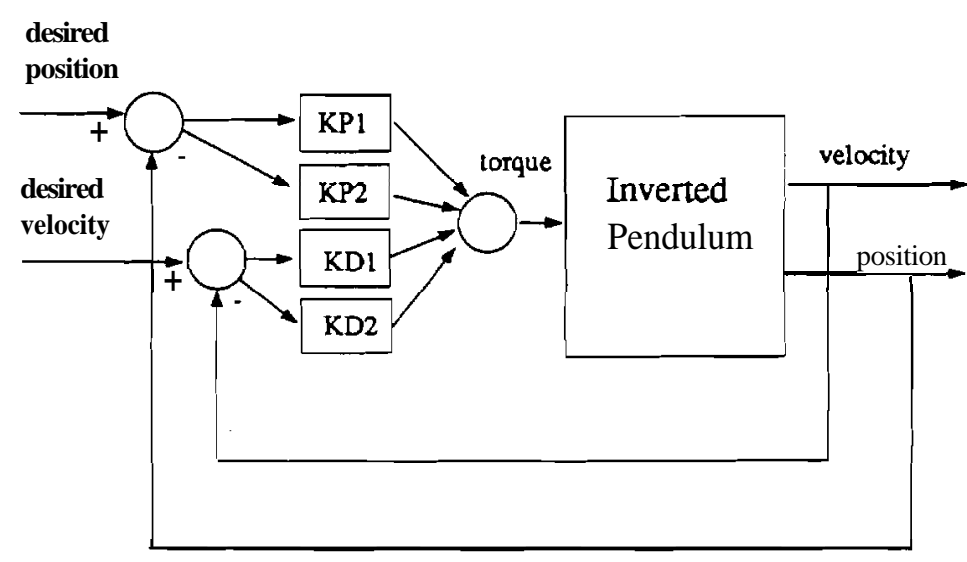

Figure 2: The PD controller with the plant

\subsection{PD Controller}

Figure 2 depicts the system with the PD controller. This scheme allows the user to disregard the internal dynamics of the system and concentrate on the input output relationship of the system from observation. The input torque to the system is a function of position and velocity errors for both links from their target states. The controller is responsible for manipulating the error data into a single appropriate command torque that is applied to the first link by the motor. The input torque $\tau$ has the following form:

$$
\tau=f\left(x_{1}, x_{2}, x_{3}, x_{4}\right)
$$

where $\left(x_{1}, x_{2}, x_{3}, x_{4}\right)$ have the same meaning and where the function $\mathrm{f}$ for a PD controller is a linear combination of the error states:

$$
\tau=K P_{1} *\left(x_{1}-x_{1 d}\right)+K P_{2} *\left(x_{2}-x_{2 d}\right)+K D_{1} *\left(x_{3}-x_{3 d}\right)+K D_{2} *\left(x_{4}-x_{4 d}\right)
$$


The coefficients $K P_{1}, K P_{2}, K D_{1}$, and $K D_{2}$ in this expression are determined by trial and error, a sometimes laborious process that is tantamount to searching for a point in four dimensional space. The difficulty associated with fine tuning the values of $K P_{2}, K D_{1}$, and $K D_{2}$ can be ameliorated by first finding the best values for the proportional constants, $K P_{1}$ and $K P_{2}$, that generate sufficient torque for the pendulum to reach the goal state and then overshoot some. Next, the derivative constants, $K D_{1}$ and $K D_{2}$, are adjusted so that the response of the system is critically damped. This procedure is somewhat ad hoc and tedious; however, the tuned parameters generate satisfactory result when applied to the system within a small range around the equilibrium point. Note that the inverted pendulum has two equilibrium points, $\theta_{2}=0^{\circ}$ and $\theta_{2}=180^{\circ}$, the former corresponding to the second link being straight up, and the latter to the straight down position. Values of these gain parameters for our experiment are presented in Section 5.

\subsection{Linear Quadratic Regulator}

We will now present another linear controller whose control law is determined by, first, a linearization of the dynamics of the motion, and, second, by the minimization of a quadratic measure of performance that has two components: an integral of the errors from the current state of the system to the goal state and the integral of the torque input required. The linearization is effected by assuming that $\theta_{2}$ is limited to a small region around the equilibrium point $x_{2}=\theta_{2}=0$, with $x_{1}=\theta_{1}$ arbitrary. We also assume that the velocities of both links, $x_{3}, x_{4}$, are relatively small, implying that all the nonlinear terms in these variables can be neglected. As a result, Eq.(3) transforms into

$$
\mathrm{x}=\mathrm{Ax}+\mathrm{B} \tau
$$

where

$$
A=\left[\begin{array}{cccc}
0 & 0 & 1 & 0 \\
0 & 0 & 0 & 1 \\
0 & -\frac{m_{2} g}{m_{1} l_{1}} & 0 & 0 \\
0 & \frac{2 m_{3} g+8 m_{2} g}{m_{1} l_{2}} & 0 & 0
\end{array}\right]
$$

and

$$
B=\left[\begin{array}{c}
0 \\
0 \\
\frac{4}{m_{1} l_{1}^{2}} \\
-\frac{8}{m_{1} l_{1} l_{2}}
\end{array}\right]
$$

Fig. 3 depicts the linearized dynamics of the system described by Eq. (4). Given the linearized dynamic equation, one may now apply any linear control strategy that is available.

We are clearly interested in determining the size of the controllable region for the above linear approximation to the nonlinear dynamic equations. This we will do in the next subsection by first bounding the approximation-induced errors in the state variables as a function of time. We will compare the state variables, as functions of time, generated by the full nonlinear model against the state variables generated by the linearized approximation. 


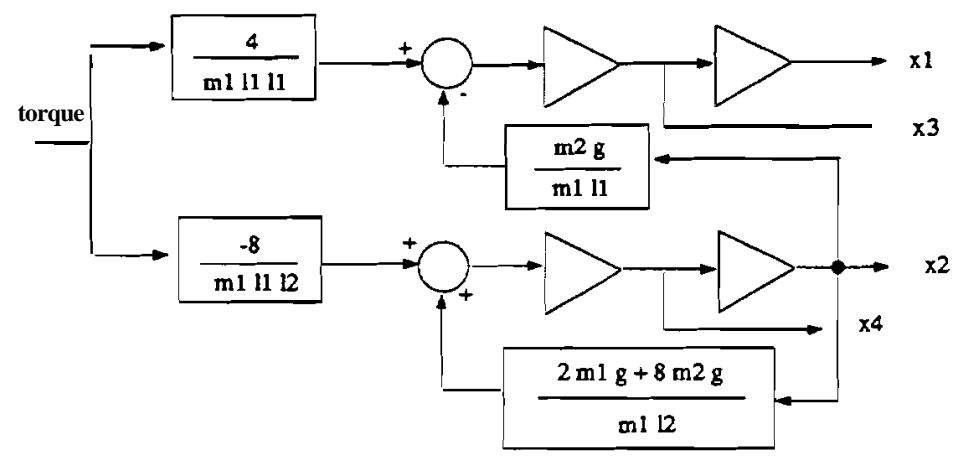

Figure 3: The Linear dynamic model

\subsubsection{Bounding the Error}

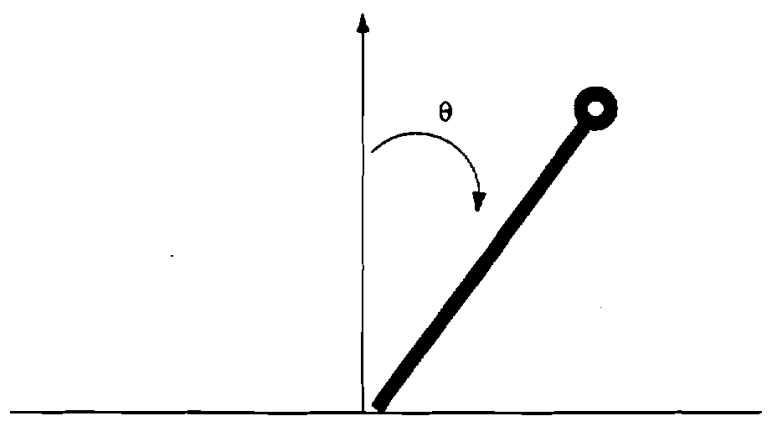

Figure 4: A simple inverted pendulum

Let's start out by assuming a one-link inverted pendulum (Fig. 4). (Presumably a one-link inverted pendulum is connected to the shaft of the motor which supplies appropriate torques to keep the link vertically upright.) The dynamic equation of the system is simply $\ddot{\theta}-a \sin \theta=0$, where $\theta$ is the angle of the link from the vertical and where a represents a constant term that is equal to gravity $g$ over the link length 1 . For now we will assume that a $\geq 1.0$. If a is less than 1.0 , we can multiply the Eq. (5) by a constant $\frac{1}{a}$ and use the constant as the variable a in the following discussion.

By designating

$$
\mathbf{x}=\left[\begin{array}{l}
x_{1} \\
x_{2}
\end{array}\right]=\left[\begin{array}{l}
\theta \\
\dot{\theta}
\end{array}\right]
$$

the dynamic equation can be written as a first order differential equation:

$$
\dot{\mathrm{x}}=\left[\begin{array}{c}
\dot{x}_{1} \\
\dot{x}_{2}
\end{array}\right]=\left[\begin{array}{c}
x_{2} \\
a \sin x_{1}
\end{array}\right]
$$

The following inequality follows from the dynamic equation

$$
\|\mathbf{x}\|-a\|\mathbf{x}\| \leq\left\|\mathbf{x}_{0}\right\|
$$


where $x_{0}$ is the initial state vector and where $\|\mathbf{x}\|$ is the norm of $\mathrm{x}$, the norm here being defined simply as the magnitude of the vector at each instant of time separately. Multipling both sides by $\exp (-a t)$ and integrating with respect to time from 0 to $t$, we get

$$
\|\mathbf{x}(t)\| \leq \frac{a+1}{a}\left\|\mathbf{x}_{0}\right\| \exp (a t)-\frac{1}{a}\left\|\mathbf{x}_{0}\right\|
$$

The above inequality describes how the norm of $\mathrm{x}$ is bounded in terms of its initial state and the elapsed time. We will use this to bound the error between the linearized and the original nonlinear system responses as functions of time.

In order to compare the true value of the state vector with its linearized approximation, we define the approximation error

$$
\mathbf{w}(t)=\mathbf{x}(t)-\mathbf{z}(t)
$$

where $\mathrm{x}$ denotes the original state vector and $\mathrm{z}$ the approximated state vector.

Differentiate the error with respect to time gives $w=x-\dot{z}$ where

$$
\dot{\mathrm{x}}=\left[\begin{array}{c}
x_{2} \\
a \sin x_{1}
\end{array}\right]+\left[\begin{array}{ll}
0 & 1 \\
a & 0
\end{array}\right] \mathrm{x}-\left[\begin{array}{ll}
0 & 1 \\
a & 0
\end{array}\right] \mathrm{x}
$$

and

$$
\dot{z}=\left[\begin{array}{ll}
0 & 1 \\
a & 0
\end{array}\right] \mathbf{z}
$$

where the latter equation follows from the linear approximation to Eq. (5). The error dynamic equation becomes

$$
\begin{aligned}
\dot{\boldsymbol{w}} & =-\left[\begin{array}{ll}
0 & 1 \\
a & 0
\end{array}\right] \mathrm{z}+\left[\begin{array}{c}
x_{2} \\
a \sin x_{1}
\end{array}\right] \\
& +\left[\begin{array}{ll}
0 & 1 \\
a & 0
\end{array}\right] \mathrm{x}-\left[\begin{array}{ll}
0 & 1 \\
a & 0
\end{array}\right] \mathrm{x}
\end{aligned}
$$

Rearranging the terms gives

$$
\dot{\mathrm{w}}-\left[\begin{array}{ll}
0 & 1 \\
a & 0
\end{array}\right] \mathrm{w}=-\left[\begin{array}{ll}
0 & 1 \\
a & 0
\end{array}\right] \mathrm{x}+\left[\begin{array}{c}
x_{2} \\
a \sin x_{1}
\end{array}\right]
$$

which may be expressed more compactly as

$$
\dot{\mathrm{w}}=\mathrm{Rw}+\mathrm{P}
$$

where

$$
\mathrm{R}=\left[\begin{array}{ll}
0 & 1 \\
a & 0
\end{array}\right]
$$

and

$$
\mathrm{p}=\left[\begin{array}{c}
0 \\
a\left(\sin x_{1}-x_{1}\right)
\end{array}\right]
$$




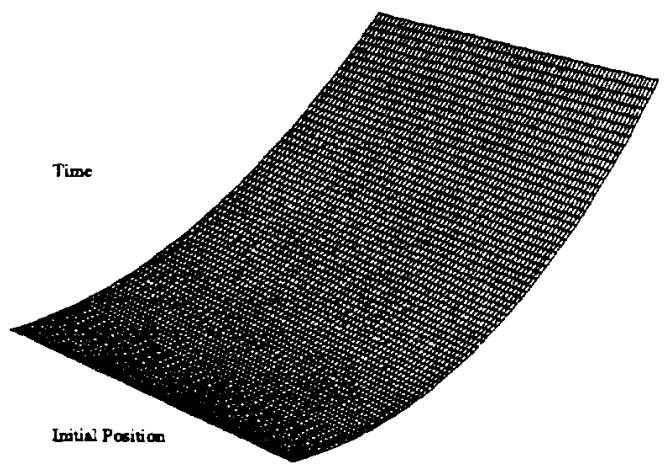

(a) position error

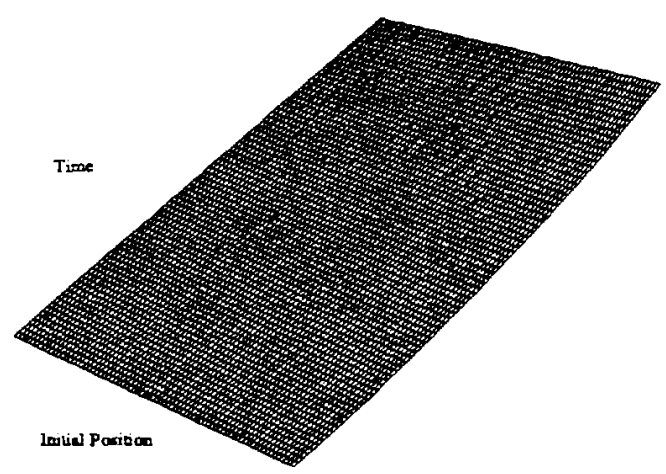

(b) velocity error

Figure 5: Bounded error profiles

The solution to Eq. (7) with the initial condition $w(0)=0$ describes the error states over time:

$$
\mathrm{w}(t)=\int_{0}^{t} \exp [R(t-\tau)] \mathrm{p}(\tau) d \tau
$$

Therefore,

$$
\begin{aligned}
|w(t)| & =|\mathbf{x}-z| \\
& =\left|\int_{0}^{t} \exp [R(t-\tau)] \mathrm{p}(\tau) d \tau\right|
\end{aligned}
$$

If we denote the two components of the vector $\mathrm{p}$ by $p_{1}$ and $p_{2}$, with $p_{1}$ of course being zero, the following bound follows from the definition of $p$

$$
p_{2} \leq a(1+\|\mathrm{x}(t)\|)
$$

Hence,

$$
\begin{aligned}
|\mathbf{w}(t)| & =\left|\int_{0}^{t} \exp [R(t-\tau)]\left[\begin{array}{c}
0 \\
p_{2}(\tau)
\end{array}\right] d \tau\right| \\
& =\left|\int_{0}^{t}\left[\begin{array}{c}
\frac{1}{\sqrt{a}} \sinh \sqrt{a}(t-\tau) p_{2}(\tau) \\
\cosh \sqrt{a}(t-\tau) p_{2}(\tau)
\end{array}\right] d \tau\right| \\
& \leq\left|\int_{0}^{t}\left[\begin{array}{c}
\frac{1}{\sqrt{a}} \sinh \sqrt{a}(t-\tau)\left\{a+(a+1)\left\|\mathrm{x}_{0}\right\| \exp a t-\left\|\mathrm{x}_{0}\right\|\right\} \\
\cosh \sqrt{a}(t-\tau)\left\{a+(a+1)\left\|\mathrm{x}_{0}\right\| \exp a t-\left\|\mathrm{x}_{0}\right\|\right\}
\end{array}\right] d \tau\right|
\end{aligned}
$$


The matrix exponential form can be solved using various methods [11]: we chose the inverse Laplace transformation method to find the above result.

The above discussions on bounding the error between the true nonlinear system and the linearized system suggest easy steps to upper-bound the difference between a linearized and a true nonlinear system for an arbitrary nonlinear system. The first step is to bound the magnitude of the state variables for the original nonlinear system in terms of the initial position and the time. The next step is to either solve the the set of differential equations for the linearized and nonlinear dynamic equations and directly bound the error or by manipulating the two dynamic equations to come up with a new error dynamic equation, as we have done. The final step is to incorporate the information of how the original state variables have traversed in time with some initial state into the bounded error equation. Of course, the procedure can be cumbersome when high order system is considered.

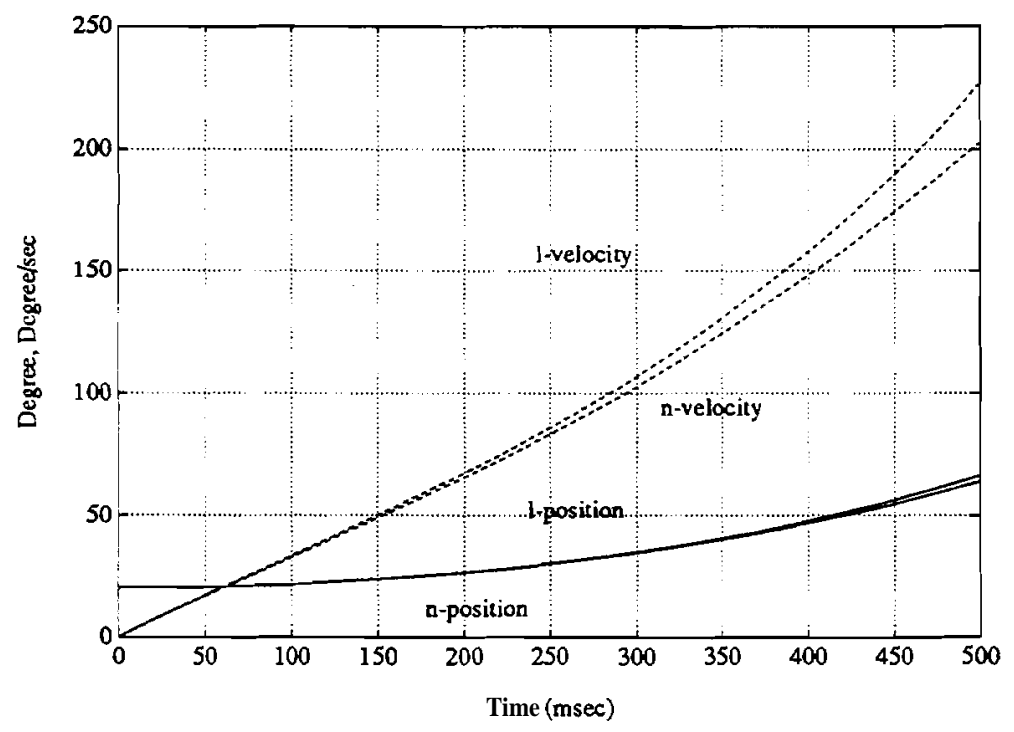

Figure 6: Trajectories of the linear and the nonlinear dynamic systems

We show in Fig. 5 a graphic depiction of the bound in Eq. (8). The surface on the left shows the bound on the position, the first component of $w(t)$, and the surface on the right on the velocity, the second component of $w(t)$. The two axes for each of the surfaces are the time and the initial position of the inverted pendulum. For generating these error surfaces, a link length 22 in $(0.56 \mathrm{~m})$ was used; this corresponds to a value of $\simeq 17.511$ for the constant a. As expected, the errors in both position and velocity grow with time for all initial positions. The same effect can also be seen by plotting the actual trajectories corresponding to the nonlinear and the linearized equations. This we have done in Fig. 6 for the initial state $x=20.0^{\prime \prime}$ and $\dot{x}=0^{\circ}$.

For both the position and the velocity trajectories, the upper curves correspond to the linearized case and the lower to the nonlinear equations. For a given initial condition, these curves help us define the controllable region in the state space. We could say that when the position and the velocity trajectories generated by the linearized model are within, 1.3 and 4.2 percent of true 


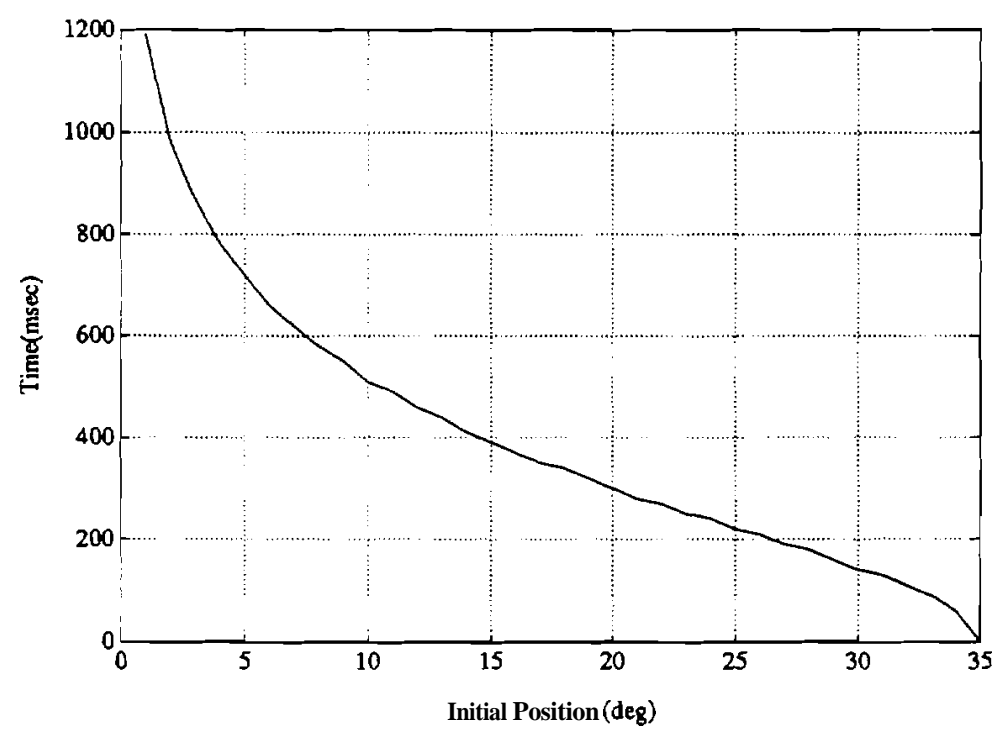

Figure 7: Available time interval versus initial position.

trajectories, respectively, as generated by the nonlinear model, the linear control would prevail and bring the system back to its equilibrium position. Based on such a criterion, the controllable region in the $(x, \mathrm{x})$ space ranges from $-35.4^{\prime}$ to $35.4^{\prime}$ for $\mathrm{x}$ and $-106 . \mathrm{S}^{\circ} / \mathrm{s}$ to $106 . \mathrm{S}^{\mathrm{G}} / \mathrm{s}$ for $\mathrm{x}$. For a given initial position, Fig. 6 also gives us the time interval during which the controller must act if linearized approximations are used for determining the control input. Again using the same criterion, Fig. 6 says that when the initial state is given by $\mathrm{x}=20.0^{\circ}$ and $\dot{x}=0^{\circ}$, the system has only 0.31 seconds in which a linear controller must act. After this time, the discrepancy between the linear and the true nonlinear models is too great and the linearized approximation becomes inapplicable. In Fig. 7, we have plotted available time for control input as a function of the initial position, assuming in all cases that the initial velocity is zero.'

\subsubsection{Linear Observer}

We will now go back to the case of the two-link inverted pendulum. Let's assume that! as is often the case in practice, the velocity measurements, $x_{3}$ and $x_{4}$, are not available. If we now denote what is actually observed by a two-dimensional vector $y$, we have the following relationship between $\mathrm{y}$ and the state vector $\mathbf{x}$ :

'The reader might question our use of the 1.3 and 4.2 percent criterion for ascertaining the extent of the controllable region for the linearized controller. For the two-link inverted pendulum we have empirically verified that when the linearization errors for the position variable is less than 1.3 percent and the linearization error for the velocity variable is less than 4.2 percent, a linear controller is applicable. 


$$
\mathrm{y}=\mathrm{Cx}=\left[\begin{array}{llll}
1 & 0 & 0 & 0 \\
0 & 1 & 0 & 0
\end{array}\right]\left[\begin{array}{l}
x_{1} \\
x_{2} \\
x_{3} \\
x_{4}
\end{array}\right]
$$

Even if the velocities are not measured, they cannot be ignored simply because the velocity terms have a fundamental role to play in the dynamics of the system. Therefore, the velocity terms must be estimated if they are not actually measured. In the linearized model, the estimated full state vector., denoted $\hat{x}$, can be found by solving the following differential equation

$$
\dot{\hat{\mathbf{x}}}=A \hat{\mathbf{x}}+B \tau+K(y-C \hat{x})
$$

which follows from Eqs. (4) and (9) by using estimation principles first promulgated by Luenberger [18]. The matrix $\mathrm{K}$ is called the gain matrix and is computed by maximizing the stability which minimizes the response time of the system, as we will discuss below. If we denote the estimation error by $\boldsymbol{e}=\mathbf{x}-\hat{\mathbf{x}}$, we get from Eq. (4) and (10)

$$
\dot{\mathrm{e}}=\dot{x}-\dot{\hat{\mathbf{x}}}=(\mathrm{A}-\mathrm{KC}) \mathbf{e}
$$

The choice of the gain matrix is dictated by the following theorem and lemma. ${ }^{2}$

Theorem [37]: Pair (A, B) is controllable if and only if for every symmetric set A of $\mathrm{n}$ complex numbers, where $\mathrm{n}$ is the number of degrees of freedom of the dynamic system, there exists a matrix $F$ such that $a(A+B F)=\Lambda$ where $a$, called the spectrum, computes the eigenvalues of its argument.

\footnotetext{
${ }^{2}$ For the benefit of those robotics readers whose background is not steeped in control theory, we will now quickly define what we mean by controllability and observability of a linear dynamic system described by $\mathrm{x}=A x+\mathrm{B} \tau$ and $\boldsymbol{y}=\mathrm{Cx}$. We say a dynamic system is controllable if by examining the initial state and some final state we can figure out whether or not there exists an input that will allow the system state to transition from one to the other in finite time. Controllability is a function of the matrix pair $(A, B)$. We say a dynamic system is observable if it is possible to cornpute every vector state from the input and what is observed, the vector $\mathrm{y}$ in our case as recorded over time. The observability turns out to be a function of the matrix pair $(A, \mathbf{C})$. It is important to note that whenever the pair $(A, B)$ is controllable, the pair $\left(A^{T}, B^{T}\right)$ is observable. By the same token, when the pair $(A, C)$ is observable, the pair $\left(\mathrm{A}^{T}, \mathbf{C}^{T}\right)$ is controllable. For the $\mathrm{A}, \mathrm{B}$, and $\mathrm{C}$ applicable to Eqs. (4) and (9), the observability of the pair $(A, C)$ is easily established by showing that the observability matrix as given by
}

$$
\left[\begin{array}{c}
\mathrm{C} \\
\mathrm{CA} \\
\mathrm{CA}^{2} \\
\vdots \\
\mathrm{CA}^{n-1}
\end{array}\right]
$$

has full rank. We can similarly establish the controllability of the pair $(A, B)$ by showing the full rank of the controllability matrix $\left[B A B A^{2} B \ldots A^{n-1} B\right]$. 
Lemma [37]: Let $(\mathrm{A}, \mathrm{B})$ be a controllable pair. Then, for any $\mathrm{n}$ dimensional vector $\mathrm{b} \in \mathrm{B}$ there exists feedback $\mathrm{F}: \mathrm{X} \rightarrow \tau$ such that the pair $(\mathrm{A}+\mathrm{BF}, \mathrm{b})$ is controllable. $\mathrm{B}, \mathrm{X}$, and $\tau$ are, respectively, the spaces spanned by the columns of the $B$ matrix, by the state vector, and by the input vector. ${ }^{3}$

To specify the gain matrix $K$, we first note that since the transpose of a matrix has the same spectrum as the original matrix, the following dynamic system is equivalent to that described by Eq. (11):

$$
\dot{e}=\left(A^{T}-C^{T} K^{T}\right) e
$$

which may in turn be written as

$$
\dot{e}=A^{T} e+C^{T} \mathrm{u}
$$

where $u$ is the state feedback $u=-K^{T} e$. The dynamic system described by this equation may now be subject to the theorem and the lemma stated above with $A=A^{T}$, and $B=C^{T}$.

Since the pair $(A, C)$ is observable for the original dynamic equations Eq. (4) and Eq. (9), the pair $\left(\mathrm{A}^{\mathrm{T}}, \mathrm{C}^{T}\right)$ is controllable. While the lemma above allows us to transform a multi-input system into a single input system, and the theorem guarantees that there exists an appropriate feedback which will place the poles of the system $\left(\mathrm{A}^{T}-\mathrm{C}^{T} \mathrm{~K}^{T}\right)$ at a set of designated locations in the open left half space of the s-plane. Using the theorem and the lemma, one can find the feedback $F$ such that $\sigma\left(\mathrm{A}^{T}+\mathrm{C}^{T} \mathrm{~F}\right)=\Lambda$ for whatever choice of $\Lambda$ we desire. In our case, the choice of $\mathrm{K}=-\mathrm{F}^{T}$ allows us to place the poles at designated locations. For detail on how to compute $\mathrm{K}$, we refer the reader to Appendix A. Note that in selecting the pole locations, the choice of $\Lambda$ determines how fast the estimate $\hat{x}$ converges to the true state $x$. Fig. 8 depicts the system with the linear quadratic controller using the observer described above. The module $-G$ in Fig. 8 represents the linear quadratic gain control which is the subject of the next subsection.

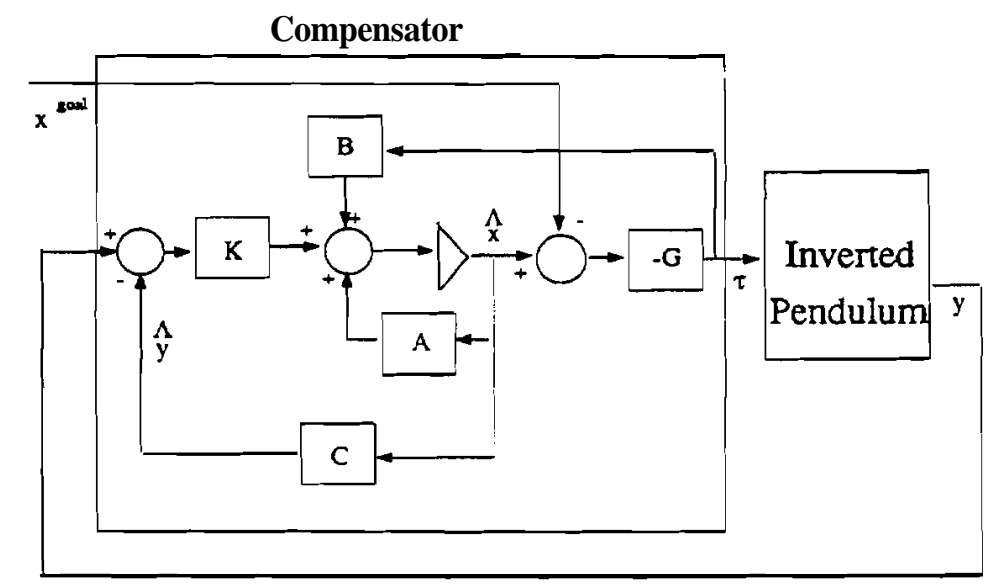

Figure 8: The Linear Quadratic Controller using a linear observer

\footnotetext{
${ }^{3}$ The lemma allows one to transform a multi-input system to an equivalent form with just one scalar input, which makes the process of finding the feedback $F$ easier. For proofs of the theorem and lemma, we direct readers to the referred text.
} 
$\mathrm{pack} / \mathrm{meng} / \mathrm{kak}$

\subsubsection{Linear Quadratic Control}

While the previous subsection showed how to compute an estimate $\hat{\mathbf{x}}$ of the full state vector $\mathrm{x}$, we will now address the issue of what exactly the control input should be. Evidently, for a linear controller the control input will be linear function of $\hat{x}$. Stated more generically, we now wish to derive a control law of the form $\tau=-G\left(\hat{\mathbf{x}}-\mathrm{x}^{g \circ a l}\right)$ that would give us the input torque $\tau$ when the state vector is $\mathbf{x}$; $G$ is called the feedback gain of the controller.

The control law is derived by minimizing the following quadratic performance criterion:

$$
\mathrm{V}=\int_{T_{\text {initial }}}^{T_{\text {final }}}\left[\left(\hat{\mathbf{x}}(t)-\mathrm{x}^{g \circ a l}(t)\right)^{T} \mathrm{Q}\left(\hat{\mathbf{x}}(t)-\mathrm{x}^{g \circ a l}(t)\right)+\mathrm{r}^{2}(t)\right] d t
$$

where the user-specified matrix $Q$ is symmetric and positive definite and the scalar value $\mathbf{r}$ is positive. The integration is with respect to time from the initial time, $T_{\text {initial, }}$, to the final time, $T_{\text {final }}$ at which time the state is expected to have reached the goal state. Note that the second integrand is proportional to the total torque needed by the system to reach the goal state.

In accordance with the derivation shown in the Appendix B, the minimization of the quadratic form can be accomplished by first solving the following Riccati equation:

$$
-\dot{\overline{\mathrm{K}}}=\overline{\mathrm{K}} \mathrm{A}+\mathrm{A}^{T} \overline{\mathrm{K}}-\overline{\mathrm{K}} \mathrm{Br}{ }^{-1} \mathrm{~B}^{T} \overline{\mathrm{K}}+\mathrm{Q}
$$

and then substituting the solution in $G=r^{-1} B^{T} \bar{K}$. The Riccati equation is a set of first order coupled differential equations that, except for very simple cases, must be solved numerically, especially so for arbitrary $T_{\text {final }}$. The equation is usually solved by considering the state trajectory backward in time. If a system is known to be controllable - as is indeed the case with our system - we may argue that, as $T_{\text {final }}$ approaches infinity, the time derivatives in the Riccati equation go to zero. As a result, the set of coupled first order differential equations become a set of linear coupled equations that may be solved by any of standard methods. $4 \mathrm{~s}$ discussed in Section 4, in our case we had to solve a set of ten coupled linear equations to find the gain matrix.

Note that by structuring appropriately the positive-definite matrix $Q$, it is possible to assign different relative weights to the different state variables. For example, for the case of controlling a two-link inverted pendulum, it is an experimental imperative that the variables $x_{2}$ and $x_{4}$ get higher priority in the determination of the control input. The reason for this is intuitively obvious. For the pendulum to be stable, the values of the variables $x_{2}$ and $x_{4}$, standing for the angular position and the angular velocity of the second link, must be brought close to zero as expeditiously as possible and then maintained there. As a secondary priority, the control input must be determined by the goal values of the other two variables, $x_{1}$ and $x_{3}$. The different priority levels assigned to the different state variables are reflected in the matrix $Q$.

While the specification of the matrix $Q$ is important in its own right, also important is the "magnitude" of $Q$ in relation to the magnitude of $r$. If the relative magnitude of matrix $Q$ is large, 
the control input, meaning the torque applied by the motor to the first link, will need to become large even for small deviations from the goal state, which may not always be possible to bring about since in real situation there is always a limit on the size of the control input. Besides, when the control input is large although within the range of what can be supplied by the motor, other factors - quantization effects, dynamic friction, motor inertia, etc. - that are difficult to model theoretically have a bearing on the stability of the sytem. On the other hand, if the magnitude of $\mathbf{r}$ is large in relation to the magnitude of the matrix $\mathbf{Q}$, the minimization discussed in this section might become overly sensitive to reducing the integral of the control input, thereby generating inadequate control inputs to drive the system to its goal state. In the section on experimental results, we have discussed how we arrived at our choices for $\mathbf{Q}$ and $\mathbf{r}$.

\subsection{Neural Controller}

The architecture of the neural controller for the system is depicted in Fig. 9. The input to the neural network consists of the four variables:

$$
\begin{aligned}
& \xi_{1}=x_{1}-x_{1}^{\text {goal }} \\
& \xi_{2}=x_{2}-x_{2}^{\text {goal }} \\
& \mathrm{f} 3=x_{3}-x_{3}^{\text {goal }} \\
& \xi_{4}=x_{4}-x_{4}^{\text {goal }}
\end{aligned}
$$

where $x_{1}, x_{2}, x_{3}, x_{4}$, the state variables defined in Section $\mathbf{3}$, are the two joint angles and the two angular velocities, and where $x_{1}^{\text {goal }}, x_{2}^{\text {goal }}, x_{3}^{\text {goal }}, x_{4}^{\text {goal }}$ designate the goal state. The output of the neural network is, except for a normalization factor, the value of the torque that the motor must produce. ${ }^{4}$ As is the case when neural networks are used for control, as opposed to when they are used for pattern classification, the reader will notice that the output of the neural network is not constrained to be discrete; the range of the output is continuous and used as such. The output activation function used at each node $y$ is the sigmoid function:

$$
f(y)=\frac{1}{1+\exp \left(-\sum w_{i} x_{i}\right)}
$$

where $x_{i}$ 's are the outputs of the previous layer and $\mathrm{w}_{\mathrm{i}}{ }^{\prime} \mathrm{s}$ the connection weights between the previous layer nodes, $x_{i}$ 's and the current node $y$. The number of nodes in the second hidden layer is three times the number used in the first hidden layer. As is usual, the bias nodes are included to shift the domain of the sigmoid function appropriately.

This neural controller is similar to the one used by Sekiguchi et al. [32], except for the fact that we have used a different number of hidden layers and node connections. Ours is a feedforward

\footnotetext{
${ }^{4}$ The motor shown in Fig. 10 is operated in the torque mode. In this mode, the output torque is proportional to the voltage applied to the motor controller. Therefore, the output of the neural network is treated as the normalized value of this voltage.
} 
neural network, which implies that the data signals only propagate in the forward direction and there is no feedback loop within the controller. On the basis of results recently proved by Cybenko [7], Funahashi [9] and others, it is interesting to note that any continuous mapping function $\left(\xi_{1}, \xi_{2}, \xi_{3}, \xi_{4}\right): \rightarrow \tau$ may be generated by a neural network with a single hidden layer whose output activation functions are sigmoid functions and provided no constraints are placed on the number of nodes in the hidden layer and on the range of weight values associated with the connections between the nodes. Nonetheless, practical considerations concerning the aforementioned constraints dictate that at least two hidden layers be used for a general application of the neural controller.

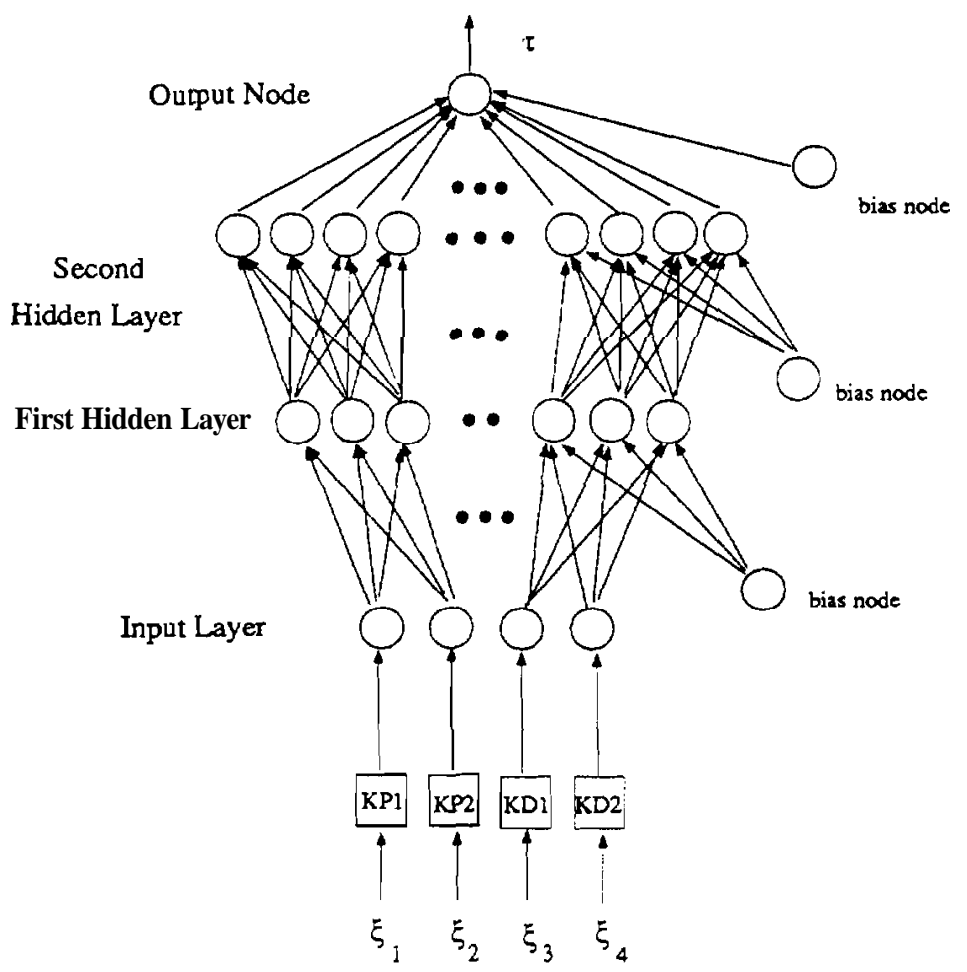

Figure 9: The Neural network Controller

As was mentioned before, a controller for a two-link inverted pendulum must not only maintain the second link vertically upright, but must also move the first link to a prespecified position. To satisfy these two goals at the same time requires that we "program" into the neural network a behavior that is best explained with the help of Fig. 10. The dashed line in (a) is the goal position of the link 1. When link 1 is, say, to the left of the goal position, as shown in (a) and when link 2 is not leaning toward the goal position of link 1. it is imperative that the torque applied by the motor to link 1 be such that the angle $\theta_{2}$ immediately assumes a value that would make the second link lean toward the goal position of link 1 . The important point to note here is that for link 1 to be "dragged" to its goal position, link 2 must remain leaning in the direction of the motion of link 1; it is simply not possible to move link 1 to its goal position if link 2 is leaning away from the direction of motion as that would cause link 2 to fall. The same is true when link 1 is to the right of the goal position, as in (b). Said equivalently, when $\theta_{1}-\theta_{1}^{\text {goal }}$ is positive. meaning when link 1 is to the right of the goal position for this link, we want the torque $\tau$ to be such that the 


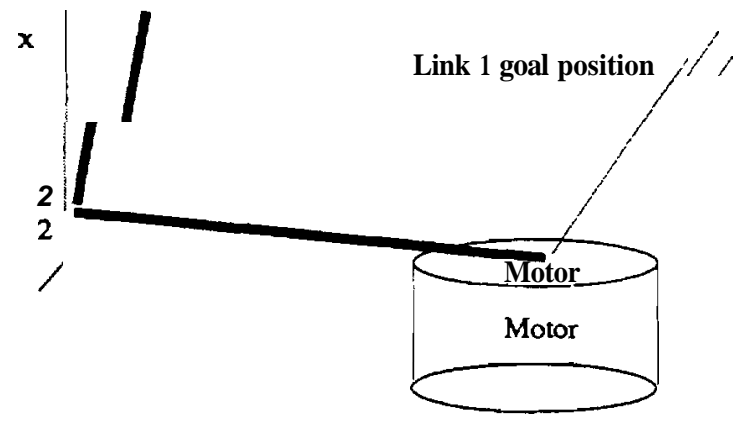

(a) link 1 is to the left of its goal position

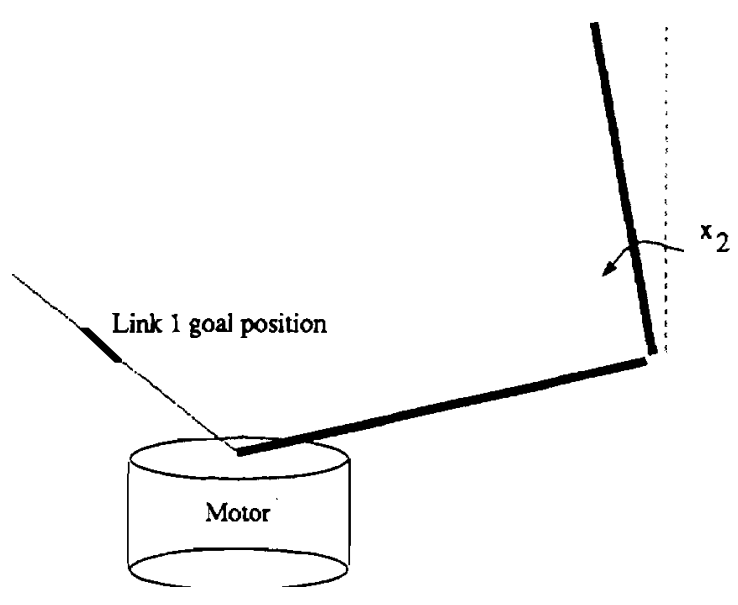

(b) link 1 is to the right of its goal position

Figure 10: Desired behaviors

change induced in $\theta_{2}, \delta \theta_{2}$, is negative. ${ }^{5}$ Similarly, when $\theta_{1}-\theta_{1}^{\text {goal }}$ is negative, we want the torque $\tau$ to be such that the change induced in $\theta_{2}$ is positive.

The question then becomes one of training the neural network so that it exhibits this behavior and that brings us to the learning phase of the neural controller. From the preceding discussion, it is apparent that the desired behavior may be quantified by prescribing a certain relation between the different components of the state vector. For example, the following relation between $x_{1}=\theta_{1}$ and $x_{2}=\theta_{2}$, used first in [32]

$$
x_{2}=x_{2 \max }\left\{\frac{2}{1+\exp \left(x_{1}-x_{1}^{g \circ a l}\right)}-1\right\}
$$

is consistent with the desired behavior, in the sense that it forces the desired correspondence between an essentially unbounded $\theta_{1}$ and a bounded and opposite $\theta_{2}, x_{2}$ being bounded by the interval $\left\{-x_{2 \max }, x_{2 \max }\right\} . x_{1}^{\text {goal }}$ is the goal position of link 1 (Fig. 11). Clearly, this is not the only possible relation that is capable of establishing such a correspondence. Moreover, it is not even clear how, given a choice of such behavior inducing functions, one would go about choosing the best one. A discussion of such questions is outside the scope of the present paper. By taking a derivative of both sides of the above relation, a similar constraint between the velocities $x_{3}$ and $x_{4}$ can be derived:

$$
x_{4}=x_{2 \max } x_{3}\left\{\frac{-2 \exp \left(x_{1}-x_{1}^{\text {goal }}\right)}{\left(1+\exp \left(x_{1}-x_{1}^{\text {goal }}\right)\right)^{2}}\right\}
$$

\footnotetext{
${ }^{5}$ This statement is only true when both $\theta_{1}$ and $\theta_{2}$ are considered positive in the direction of clockwise motion from the standpoint of an observer at the origin sitting on top of the motor.
} 
Now that we know how to translate the desired behavior into a pair of relations between the state variables, we must next address the question of how to "embed" these relations in the neural controller, which takes us to the learning phase for calculating the connection weights. Evidently, the connection weights must be consistent with the two relations we have shown above. The actual learning takes place in two phases as follows:

\section{PHASE 1:}

At the very outset, the motor does not apply any torque to link 1, and, a human operator drops link 2 first to the left and then to the right. During these free falls and until $x_{2}$ reaches $|45|^{0}$ on either side, the values of the 4-tuple $\left(x_{1}, x_{2}, x_{3}, x_{4}\right)$ is recorded. Usually, seventy sets of 4-tuples are recorded on either side. ${ }^{6}$

Clearly, these values will not conform to the relations shown previously. So, treating $x_{1}$ and $x_{3}$ as independent variables, we compute the desired

$$
\begin{aligned}
x_{2}^{\text {desired }} & =x_{2 \max }\left\{\frac{2}{1+\exp \left(x_{1}-x_{1}^{\text {goal }}\right)}-1\right\} \\
x_{4}^{\text {desired }} & =x_{2 \max } x_{3}\left\{\frac{-2 \exp \left(x_{1}-x_{1}^{\text {goal }}\right)}{\left(1+\exp \left(x_{1}-x_{1}^{\text {goal }}\right)\right)^{2}}\right\}
\end{aligned}
$$

Now the Reinforcement Module computes a measure of the difference between the actual recorded values of the dependent variables $x_{2}$ and $x_{4}$ and their desired values:

$$
\delta \tau=-C *\left\{\mathrm{~K}_{1}\left(x_{2}^{\text {desired }}-x_{2}\right)+\mathrm{K}_{2}\left(x_{4}^{\text {desired }}-x_{4}\right)\right\}
$$

where the constants $K_{1}$ and $K_{2}$ may be used to give different weights to the two types of errors. In our implementation, they are both set to 1 . Except for the normalization constant $\mathrm{C}$, the left hand side may be construed to be proportional to the torque error that the neural controller must ask the motor to correct so that the behavior of the neural controller will be in compliance with the desired behavior. The error torque $\delta \tau$ is then backpropagated over the neural network and the connection weights modified in accordance with the pseudo-impedance method [29]. Initially, the connection weights are assumed to be random numbers ranging between $(-1,1)$. The pseudoimpedance method is a modification of the common hackpropagation algorithm for learning, in that, for each internode connection, the previous two values are simultaneously taken into account during the modification process. From a more theoretical standpoint, the usual backpropagation learning implements steepest descent, using the gradient of the output error, in the space of

\footnotetext{
${ }^{6}$ Each 4-tuple is recorded by the computer interrogating a decoder and this process takes place irregularly, although at a rapid rate compared to the time constants associated with the motions of the inverted pendulum. On the average, the time interval between successive readings of the 4-tuples is $15 \mathrm{msec}$.
} 
connection weights, but, as is the case with all steepest descent methods, can get trapped in a local minimum. On the other hand, the pseudo-impedance method models the rate change in the connection weights as a second order damped system, similar to a mechanical vibration system. As a result, the change in each connection weight during learning is a function of the current gradient of error and the two previous values for the same connection weight. The advantage of the pseudo-impedance method lies in making the learning process more immune to getting trapped in the local minima in the space of connection weights. In what follows, when we refer to learning by backpropagation, we will mean backpropagation using the pseudo-impedance approach.

The backpropagation process is repeated for each four tuple $x_{1}, x_{2}, x_{3}, x_{4}$ recorded during the two free falls of link 2. Of course, the forward computations during the learning algorithm use the error state variables $\left(\xi_{1}, \xi_{2}, \xi_{3}, \xi_{4}\right)$. As we will discuss in greater detail in the Results section, the backpropagation is carried out five times for each recorded four tuple. A learning cycle consists of traversing through the entire data set collected during the two free falls, with each 4-tuple processed five times, and with a 1000 passes through the data set.

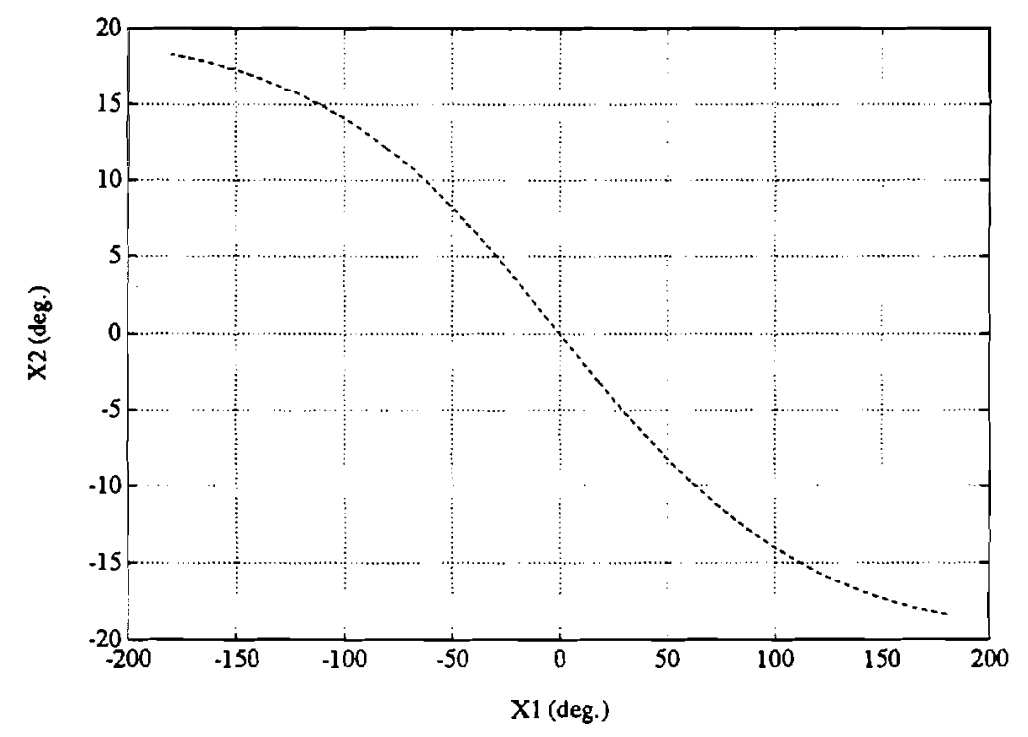

Figure 11: The desired correspondence between $\theta_{1}$ and $\theta_{2}$

\section{PHASE 2:}

After the initial training with the two free falls is complete, the neural controller with the updated weight values is used to control the inverted pendulum. What that means is that the neural controller now generates a. voltage value that is applied to the motor controller and the motor applied a torque to link 1 in proportion to the applied voltage. Since the learning so far only involved the free falls, the controller at this stage will not possess adequate competence and link 2 will fall invariable, although with decreased frequency as the link weights get refined by each training cycle. When link 2 does fall, as was the case in Phase 1, the values of the 4-tuple $\left(x_{1}, x_{2}, x_{3}, x_{4}\right)$ are recorded until the the angle that link 2 subtends with the vertical exceeds $45^{\circ}$ on either side. Using the same rationale as in Phase 1 , the desired torque is computed again 
by using Eq. 19. Let the torque produced by the motor at the instant a 4-tuple is recorded be denoted $\tau^{\text {motor }}$. (Recall, this is the torque that is the output of the neural controller. We are assuming that the motor is able to produce its commanded torque exactly.) The Reinforcement Module then computes the correcting torque value, $\delta \tau$, given in Eq. (19) and this correcting torque is backpropagated over the neural network and the connection weights modified using the pseudo-impedance procedure as before.

The need for a large number of learning cycles by the backpropagation algorithm can easily translate into a human operator having to lift link 2 manually hundreds of times to its upright position and letting the neural network control it while the 4-tuples are being recorded. This can be onerous, if not downright impractical. We have therefore devised a computer simulation of the inverted pendulum. This computer simulation allows us to carry out much of Phase 2 learning by simulation. Since a simulation cannot capture all the imponderables associated with an actual system, a simulated Phase 2 learning must still be followed by learning on the actual system. With each learning cycle consisting of 1000 passes through a set of 4 -tuples recorded during a single fall, each tuple processed five times, the simulated part of Phase 2 learning usually consists of 300 such cycles involving an identical number of controlled falls. Subsequently, in actual experimentation, the 4-tuples collected during another 50 to 100 controlled falls are used to fine-tune the connection weights. After this rather elaborate and lengthy learning process, to take care of day-to-day variations induced by temperature, wear and tear, and other effects, Phase 2 learning is executed four or five times before each demonstration of the inverted pendulum.

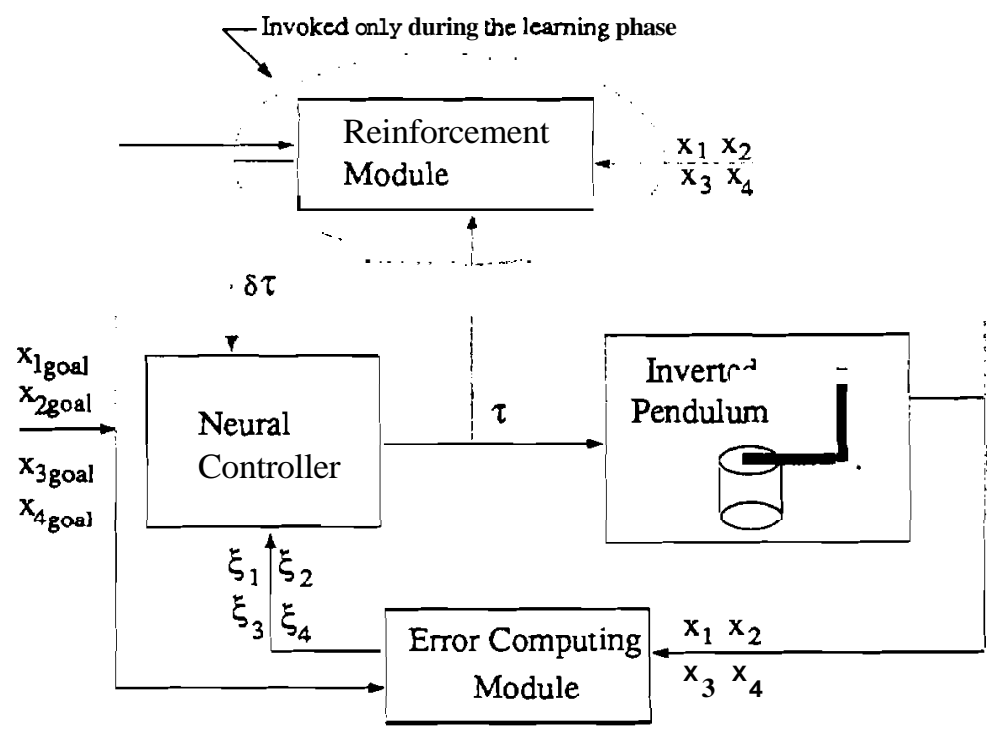

Figure 12: The flow of control using the neural controller 


\subsection{Nonlinear Controller}

\subsubsection{Preliminaries}

The neural controller we discussed in the preceding section was a nonlinear controller. While a neural controller is convenient to use, it suppresses the nature of the dependence of the motorgenerated torque on the measured values of the state vector that are fed back. While in most practical situations, it probably is not necessary to understand the exact nature of this dependence; nevertheless, there are valid intellectual reasons for the contrary. In this section, we will pursue a different approach and use the dynamic equations of the system to derive a nonlinear controller. In contrast with the neural controller, the controller presented here will tell us how precisely the motor-generated torque depends on the evolving state vector. In order to derive this nonlinear controller, we will rewrite Eq. (3) as follows

$$
\left[\begin{array}{c}
\dot{x_{1}} \\
\dot{x_{2}} \\
\dot{x_{3}} \\
\dot{x_{4}}
\end{array}\right]=\left[\begin{array}{c}
x_{3} \\
x_{4} \\
f_{3} \\
f_{4}
\end{array}\right]+\left[\begin{array}{c}
0 \\
0 \\
\frac{m_{2} l_{2}^{2}}{4 I_{2}} \\
-\frac{m_{2} l_{1} \cos x_{2}}{2 \mathrm{I}}
\end{array}\right] \tau
$$

where

$$
\begin{gathered}
\mathrm{f} 3=\frac{m_{2}^{2} l_{2}^{2} \sin x_{2}}{8 \mathrm{I}}{ }^{*}\left(l_{2}^{2} x_{3} x_{4} \cos x_{2}-l_{1} l_{2} x_{4}^{2}+l_{1} l_{2} x_{4}^{2} \cos ^{2} x_{2}+2 l_{1} g \cos x_{2}\right) \\
f_{4}=\frac{m_{2} l_{1} l_{2} \sin x_{2}}{16 \mathrm{I}} *\left(4 m_{2} l_{2}^{2} x_{3} x_{4} \cos ^{2} x_{2}-4 m_{2} l_{1} l_{2} x_{4}^{2} \cos x_{2}+m_{1} l_{1} l_{2} x_{3}^{2} \cos x_{2}+2 m_{1} l_{1} g\right. \\
\left.+8 m_{2} l_{1} g+4 m_{2} l_{1} l_{2} x_{3}^{2} \cos x_{2}+\frac{m_{2} l_{2}^{3} \sin ^{2} x_{2} \cos ^{2} x_{3}}{l_{1}}+\frac{2 m_{2} l_{2}^{2} g \sin ^{2} x_{2}}{l_{1}}\right)
\end{gathered}
$$

and

$$
I=\left(\frac{m_{1} m_{2}}{16}+\frac{m_{2}^{2}}{4}\right) l_{1}^{2} l_{2}^{2}+\frac{m_{2}^{2} l_{2}^{4} \sin ^{2} x_{2}}{16}-\frac{m_{2}^{2} l_{1}^{2} l_{2}^{2} \cos ^{2} x_{2}}{4}
$$

Such an equation is expressed more generally as

$$
\dot{x}=f(x)+\sum_{1}^{n} g_{i}(x) \tau_{i}
$$

where $f(x)$ is called the drift term, since in the absence of any applied input the state would drift in accordance with this function, and where each $g_{i}(x)$ is the active control term that dictates how $i^{\text {th }}$ component of the input influences the state. The advantage to writing the nonlinear equation, Eq. (3), in this form is that it makes explicit the part that is responsible for the inputindependent change in the state vector and the part that is directly dependent on the forcing input. The functions $\mathrm{f}(\mathrm{x})$ and $\boldsymbol{g}_{i}(\mathbf{x})$ may be thought of as vector fields, particularly so in light of our discussion to follow. 
Note that the task of a nonlinear controller is to transform the current system state $\mathbf{x}$ into the desired goal state $\mathrm{x}^{\text {goal }}$ in finite time by invoking appropriate values for the inputs $\tau_{i}$ 's. Evidently, in order for a nonlinear controller to accomplish this task, the system must be controllable, in a sense different from what was discussed in Section 4.2.2, since now we wish to include some measure of the nonlinearities. As will be shown here, the weak controllability of a nonlinear system is determined by the rank of the nonlinear controllability matrix that will be defined shortly. We first introduce some notations and definitions.

There are three well known distinct Lie derivatives we need to consider. The first one is a derivative of a vector field with respect to another vector field. This type of derivative is often called the Lie bracket. The Lie bracket produces another vector field and is defined as

$$
[f, g]=\frac{\partial f}{\partial x} g-\frac{\partial g}{\partial x} f
$$

where both $\mathrm{f}$ and $\mathrm{g}$ are vector fields defined on $\mathrm{R}^{\mathrm{n}}$. For multiple nested forms, the definition of a Lie bracket is similar to what is shown in Eq. (21) with $\mathrm{g}$ replaced by a vector field that is the result of a single or nested Lie bracket(s). Nested Lie brackets are shown more conveniently by using the following notation:

$$
\begin{gathered}
g=\left(a d^{0} \mathbf{f}, \mathrm{g}\right) \\
{[f, g]=\left(a d^{1} \mathbf{f}, \mathrm{g}\right)} \\
{[\mathrm{f},[\mathrm{f}, \ldots[\mathbf{f}, \mathrm{g}]]]=\left(a d^{n} \mathbf{f}, \mathrm{g}\right)}
\end{gathered}
$$

We now define the nonlinear controllability matrix as

$$
\mathcal{C}=\left[(\operatorname{ado} f, g),\left(a d^{1} f, g\right), \ldots\left(a d^{n-1} f, g\right)\right]
$$

where $\mathrm{n}$ denotes the dimension of the state space. If the above Lie derivatives are applied to the linear system described by Eq. (4), with $\mathrm{f}=\mathrm{Ax}$ and $\mathrm{g}=\mathrm{B}$, we have the familiar controllability matrix for a linear system

$$
\begin{aligned}
\mathcal{C} & =\left[\left(a d^{0} \mathrm{f}, \mathrm{g}\right),\left(a d^{1} \mathrm{f}, \mathrm{g}\right), \ldots\left(a d^{n-1} \mathrm{f}, \mathrm{g}\right)\right] \\
& =\left[B, A B, A^{2} B \ldots A^{n-1} B\right]
\end{aligned}
$$

which was shown as a footnote in Section 4.2.2. In the linear case, if the controllability matrix is of full rank, meaning of rank $\mathrm{n}$, the system is controllable and the the system can be transformed into a controllable canonical form shown in Appendix A. A similar result can be derived for the nonlinear case, and that is the topic of the next subsection. 
The second type of the Lie derivative is of a function with respect to a vector field. Let $h$ be a function such that $\mathrm{h}: \mathrm{R}^{\mathrm{n}} \rightarrow \mathcal{R}$ and $\mathrm{h} \in \mathrm{C}^{\infty}$. The Lie derivative of $\mathrm{h}$ with respect to a vector field $\mathrm{f}$ is defined as

$$
\begin{aligned}
\mathrm{L}_{\mathrm{f}} h & =(\nabla h)^{T} \mathrm{f} \\
& =\frac{\partial h}{\partial x_{1}} f_{1}+\frac{\partial h}{\partial x_{2}} f_{2} \ldots+\frac{\partial h}{\partial x_{n}} f_{n}
\end{aligned}
$$

It is possible to apply the operator $L_{f}$ to a function a multiple number of times. Multiple application are denoted in the following manner:

$$
\begin{gathered}
\mathrm{L}_{\mathrm{f}}^{0} h=h \\
\mathrm{~L}_{\mathrm{f}}^{1} h=(\nabla h)^{T} \mathrm{f} \\
\mathrm{L}_{\mathrm{f}}^{2} h=\left(\nabla \mathrm{L}_{\mathrm{f}}^{1} h\right)^{T} \mathrm{f} \\
\mathrm{L}_{\mathrm{f}}^{n} h=\left(\nabla \mathrm{L}_{\mathrm{f}}^{n-1} h\right)^{T} \mathrm{f}
\end{gathered}
$$

The third type of the Lie derivative is of a gradient with respect to a vector field. We define the derivative as

$$
\mathrm{I}_{\mathbf{f}}(\nabla h)^{T}=f^{T}\left(\frac{\partial(\nabla h)^{T}}{\partial \mathbf{x}}\right)+(\nabla h)^{T}\left(\frac{\partial \mathbf{f}}{\partial \mathbf{x}}\right)
$$

To appreciate the usefulness of these derivatives, it is necessary to realize that the space of state vectors has a structure of its own, just as the space that is the surface of a sphere has structure. To illustrate, consider the case when our state vector is simply $\left[x_{1}, x_{3}\right]^{T}$, where $x_{1}$ is the position of the first link and $x_{3}$ the position of the second link. A moment's reflection would show that the space spanned by this state vector is $\mathrm{T}^{2}$, the surface of a torus. On the other hand, if we have only a one-link inverted pendulum, with the state vector $\left[x_{1}, x_{2}\right]^{T}$, the space spanned would be $S^{1} \mathrm{xR}^{1}$, the structure of which is a cylindrical surface. For the case of two-link inverted pendulum, one can show similarly that the space spanned by the state vector is $S^{1} \times R^{1} \times S^{1} \times R^{1}$. The realization that the space spanned by the state vector has a shape allows one to pose the question of control as one involving the calculation of a trajectory in this space from some initial point to some goal point. For example, if we again consider that our state vector is limited to $\left[x_{1}, x_{3}\right]^{T}$, we may now specify the initial and the final states by points on a torus and the problem of control is then to find a trajectory between the two points and the inputs that would steer the state along the trajectory. Questions of control posed in this manner are very similar to questions in differential geometry where there happens to a rich body of literature dealing with the issue of trajectory calculation in different types of spaces.

Now that we have established a connection between control and differential geometry, we must of necessity invoke the pertinent concepts related to the latter, such as the notion of manifolds, 
homeomorphism, diffeomorphisms, tangent spaces, etc. Manifolds obviate the need for explicating the overall structure of a space; when we say that space is a manifold, what we mean, at least informally, is that the space is locally Euclidean, as for example the surface of a torus, or the surface of a sphere. The concepts of homeomorphisms means that the mapping is one-to-one, onto, and that the mapping exists in both directions and is continuous. Diffeomorphism involves the additional constraint that the mapping must be $\mathrm{C}^{\prime \prime}$.

These concepts can now be used to give meaning to the three Lie derivatives. The first derivative, the Lie bracket, is the rate of change of $g$ in the direction of $f$. To show this, we invoke the following result derived in [4]

$$
[f, g](p)=\lim _{t \rightarrow 0} \frac{1}{t}\left[\left(\Phi_{-t}^{f}\right)_{*} \mathrm{~g}\left(\Phi_{t}^{f}(p)\right)-\mathrm{g}(p)\right]
$$

where $\Phi_{t}^{f}$ is a mapping from the manifold at point $p$ to the manifold at a "downstream" point along the integral curve for the vector field $f$; the downstream point is $t$ "units" away from $p$. The vector field $g$ is then calculated at the point in the downstream manifold that corresponds to the point $\mathrm{p}$. Subsequently, this vector is transformed back into the manifold at $\mathrm{p}$. The difference between the original $g$ and the new $g$ at $p$ yield a derivative of $g$ in the direction of $f$. More formally, the first term in the bracket is a tangent vector which is obtained by first mapping the point $\mathrm{p}$ through $\Phi_{t}^{f}$, and after evaluating the vector field $\mathrm{g}$ at the new point, the resulting vector is taken back to the tangent space at $p$ through $\left(\Phi_{-t}^{f}\right)_{*}$. Thus, we can consider the Lie bracket as the "derivative" of the vector field $g$ in the direction of the vector field $f$. The first Lie bracket generates a vector field on the tangent plane of a point on the manifold that corresponds to the state space. The higher-order Lie brackets generate vectors outside the tangent space and are useful in generating directions along the optimal trajectory of the state vector.

Simply stated, the second Lie derivative $L_{f}^{1}$ is the rate of change of a scalar field in the direction of a vector field. Similarly, the Lie derivatives defined above recursively are higher order derivatives of the scalar field in the direction of the vector field $\mathrm{f}$. The third Lie derivative is identical to the first, except for the fact that one of the vector fields is the gradient of a scalar function. The gradient of a scalar function is sometimes referred to as a covector field. The usefulness of the second and the third type of Lie derivatives will be seen in Appendix D where we will prove the following result, known as the Leibniz formula:

$$
\mathrm{L}_{[\mathrm{f}, \mathrm{g}]} h=\mathrm{L}_{\mathrm{g}} \mathrm{L}_{\mathrm{f}} h-\mathrm{L}_{\mathrm{f}} \mathrm{L}_{\mathrm{g}} h
$$

The Leibniz formula and the Lie bracket are used to derive the conditions that must be satisfied by a nonlinear system if the system is to be transformed into a nonlinear controllable canonical form. As we will show in the following subsection, when a nonlinear system is expressed in the controllable canonical form, the system becomes effectively linear by applying a nonlinearitycanceling feedback. 


\subsubsection{Transformation and Controller}

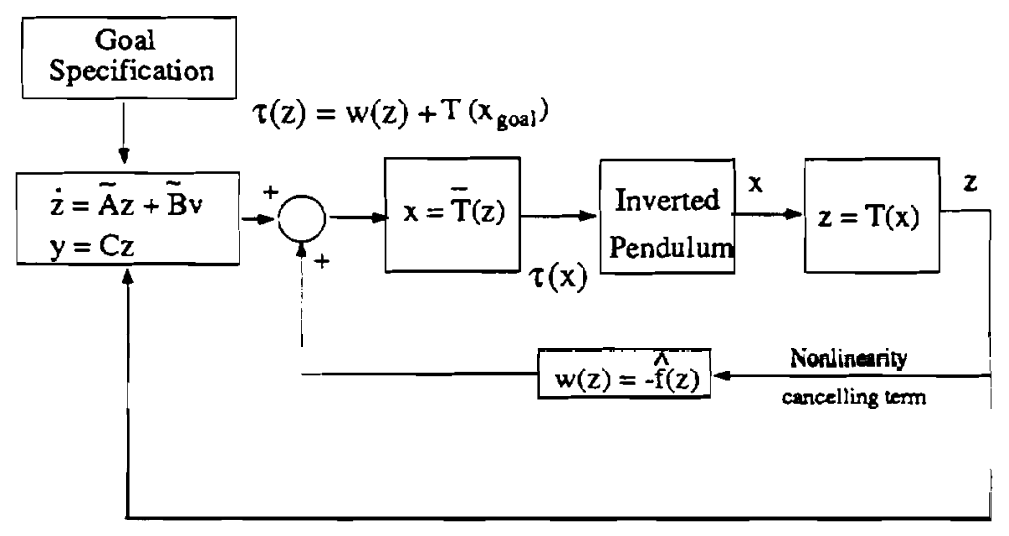

Figure 13: Plant control using the Nonlinear Controller

We confine our discussion to a single input case. The multi-input case can be generalized from the single input case. The first thing we want to do is to transform, if possible, the nonlinear equation

$$
x=f(x)+g(x) \tau
$$

into the nonlinear controllable canonical form

$$
\dot{\mathbf{z}}=\left[\begin{array}{c}
z_{2} \\
z_{3} \\
\vdots \\
z_{n} \\
\hat{\mathbf{w}}\left(z_{1}, z_{2}, \ldots, z_{n}\right)
\end{array}\right]+\left[\begin{array}{c}
0 \\
0 \\
\vdots \\
0 \\
1
\end{array}\right] \tau
$$

under some transformation $\mathbf{z}=\mathrm{T}(\mathbf{x}): \mathrm{R}^{\mathrm{n}} \rightarrow \mathrm{R}^{\mathrm{n}}$. The motivation for seeking such a transformation is inspired by Sommer [33], and the transformation, as was first pointed out by Zak and MacCarley [41], allows us to easily arrange for a feedback that would cancel the nonlinear term $\hat{\mathrm{w}}\left(z_{1}, z_{2}, \ldots, z_{n}\right)$, turning the control problem into one of linear control.

We now present the sufficient conditions, a.s shown in [41], under which a nonlinear canonical control form of Eq. (23) exists given a general nonlinear system of Eq. (22). First, assume that $\mathrm{T}$ is diffeomorphic, meaning that there exists a smooth transformation $\mathrm{z}=\mathrm{T}(\mathbf{x})$ such that its inverse $\mathbf{x}=\overline{\mathrm{I}}(\mathbf{z})$ exists and is smooth and that $\mathrm{T}(0)=0$. Differentiating $\mathbf{z}$ with respect to time and equating term by term with the Eq. (23) results in the following equation

$$
\frac{\partial \mathrm{T}_{1}}{\partial \mathbf{x}}=\left[\begin{array}{lllll}
0 & 0 & \ldots & 0 & 1
\end{array}\right] \mathcal{C}^{-1}
$$

where $T_{1}$ denotes that part of the mapping which results in the first component of $\mathbf{z}$; in other words $z_{1}=\mathrm{T}_{1}(\mathbf{x})$. For details, consult Appendix C. Equation (24) states that if the nonlinear 
controllability matrix $\mathrm{C}$ is nonsingular and the partial differential equation is solvable, we can compute the transformation $T_{1}$. Once $T_{1}$ is found we can calculate the rest of the transformation using the second type of the Lie derivative repeatedly as shown in the formentioned Appendix.

Thus, sufficient conditions for the existence of the transformation $\mathrm{T}$ are: 1) the nonlinear controllability matrix $\mathrm{C}$ has a full rank; and 2) the Frobenius theorem [23] is satisfied, that is the partial differential equation (24) is solvable.

Thus we have the following algorithm to find the diffeomorphic transformation to transform a nonlinear system into a nonlinear controllable canonical form.

\section{Form the controllability matrix $\mathrm{C}$}

2. Find its inverse and let $q$ designate the last row of the inverse matrix

3. Solve the partial differential equation $\frac{\partial \mathbf{T}_{1}}{\partial \mathbf{x}}=\mathrm{q}$

4. Construct the transformation $\mathrm{T}$

Once the dynamic system is made into a nonlinear canonical form of Eq. (23), one can use the following input $\boldsymbol{\tau}(\mathbf{x})=-\left.\hat{\mathbf{w}}(\mathbf{z})\right|_{z=T(x)}+\left.v(z)\right|_{z=T(x)}$ to control the system. The first term cancels the nonlinearity of the dynamic system and makes the system linear in the new coordinate space. Now the system can be controlled by almost any type of a linear control scheme in coordinate $\mathbf{z}$. Fig. 13 depicts the flow of control using the nonlinear controller described.

One may not be comfortable with this approach to solving a nonlinear control problem. After all, since $\mathrm{x}$ is the observation vector: in general a goal would be specified most easily in the $\mathrm{x}$ vector space. However, the approach outlined here dictates that the goal be specified in the $z$ vector space. This problem can be gotten around by first transforming the x specification of the goal state into a $\mathbf{z}$ specification by using the transformation $T$, yielding $T\left(\mathbf{x}_{\mathbf{g} \circ a \mathbf{l}}\right)$. Examination of the feedback paths dictates that if the goal state is to be recognized at the point shown in Fig. 13, the complete specification of the goal is $\mathbf{z}_{\text {goal }}=\hat{w}(\mathbf{z})+T\left(\mathbf{x}_{\text {goal }}\right)$, where $\hat{w}(\mathbf{z})$ is the nonlinearity canceling feedback in Fig. 13.

\subsection{Fuzzy Controller}

In this section, we will present a. new fuzzy-logic based architecture for controlling a two-link inverted pendulum. But, first, since fuzzy-logic based controllers are now fairly commonplace, we will review here in only the briefest possible manner the various considerations that have to go into designing such a controller.

When fuzzy logic is used for designing a system controller, the control is derived from rules that are founded more on our own intuitive understanding of the problem rather than on some detailed 
mathematical modeling. In the rules, often stated in the usual IF-THEN form, the state of the system is described by the use of deliberately vague and imprecise terms such as "small torque," "very large velocity," etc.

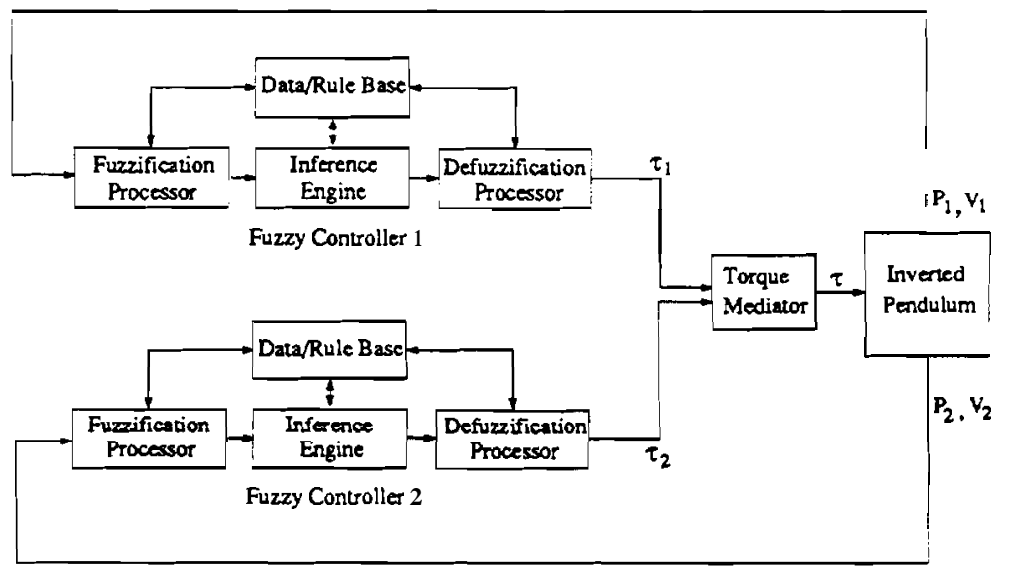

Figure 14: The system architecture with the fuzzy controller.

Therefore, the initial step is to delineate a set of linguistic variables relevant to the problem. Obviously, even if we had no access to a mathematical analysis of this system, we'd have to say that the important variables are the torque output by the motor, the positions and the velocities of the two links. Next, we must list the terms that comprise each linguistic variable. Consider, for example, our intuitive rule such as "IF the position of link 1 relative to the goal position is small in the positive direction, AND IF the velocity of link 1 is small in the negative direction, THEN the motor need output zero torque." This rule makes intuitive sense because, to satisfy the antecedent of this rule, link 1 will have to be moving toward its goal and there would be no need for the motor to apply any additional torque.

Rules like the one mentioned above indicate that linguistic variables, such as position, be comprised of terms like negative large, negative medium, e $t$ with each term being a fuzzy measure of the position of a link from the goal position. In Fig. 15, we have listed all the terms that we have used for the five linguistic variables in our system. in this figure, we have also shown the fuzzy membership functions for each of the terms.

The reader might ask, why nine terms for, say, the motor output torque and only seven for link 2 position terms? As is true for all fuzzy-logic based controllers, the justification for what is shown in Fig. 15 is purely empirical. Initially, we designed a controller with the same number of terms, first five and then seven, for each of the linguistic variables, but the results were inferior to those obtained with the terms listed in Fig. 1.5. We also implemented a version of the controller with more terms, but the resulting improvements are only marginal and not worth the increased complexity that comes into play when specifying the rules. Recall that the rules, for the most part, are supposed to capture our intuition regarding how a variable must be changed given observations regarding some other variable. When the granularity of the terms becomes too fine, it becomes more difficult to specify the rules with any measure of confidence. The membership functions shown in Fig. 15 were also arrived at empirically. Again, if the granularity of the terms is too fine, 


\begin{tabular}{|c|c|c|c|c|c|}
\hline $\begin{array}{l}\text { Linguistic } \\
\text { yariables } \\
\text { terms }\end{array}$ & $\begin{array}{l}\text { Position } 1 \\
\text { (degrees) }\end{array}$ & $\begin{array}{l}\text { Position } 2 \\
\text { (degrees) }\end{array}$ & $\begin{array}{c}\text { Velocity } 1 \\
\text { (degrees/sec) }\end{array}$ & $\begin{array}{l}\text { Velocity } 2 \\
\text { (degrees/sec) }\end{array}$ & $\begin{array}{l}\text { Torque } \\
\left(\mathrm{kg}-\mathrm{m} / \mathrm{s}^{2}\right)\end{array}$ \\
\hline \multicolumn{6}{|l|}{$\begin{array}{l}\text { Negative } \\
\text { Very }\end{array}$} \\
\hline & & & & & $\begin{array}{lll}-5 & -4.5 & -4\end{array}$ \\
\hline \multirow{2}{*}{$\begin{array}{l}\text { Negative } \\
\text { Large }\end{array}$} & & & & & \\
\hline & & $\begin{array}{lll}-45 & -30 & -20 \\
\end{array}$ & & & $-4.4-3$ \\
\hline \multirow{2}{*}{$\begin{array}{l}\text { Negative } \\
\text { Medium }\end{array}$} & & & & & \\
\hline & $\begin{array}{llll}-80 & -60 & -20\end{array}$ & $\begin{array}{lll}-24 & -18 & -12\end{array}$ & $\begin{array}{llll}-80 & -60 & -20\end{array}$ & $\begin{array}{lll}-80 & -60 & -20\end{array}$ & $-2.7 \quad-2.2 \quad-1.3$ \\
\hline \multirow{2}{*}{$\begin{array}{c}\text { Negative } \\
\text { Small }\end{array}$} & & & & & \\
\hline & $\begin{array}{lll}-30 & -15 & 0 \\
\end{array}$ & $\begin{array}{lll}-14 & -8 & -0.3 \\
\end{array}$ & $\begin{array}{lll}-30 & -15 & 0 \\
\end{array}$ & $\begin{array}{lll}-30 & -15 & 0 \\
\end{array}$ & $\begin{array}{lll}-1.6 & -0.8 & -0.2 \\
\end{array}$ \\
\hline \multirow{2}{*}{ Zero } & & & & & \\
\hline & $\begin{array}{lll}-5 & 0 & 5\end{array}$ & $\begin{array}{lll}-0.5 & 0 & 0.5\end{array}$ & $\begin{array}{lll}-10 & 0 & 10\end{array}$ & $\begin{array}{lll}-4 & 0 & 4\end{array}$ & $\begin{array}{lll}-0.4 & 0 & 0.4\end{array}$ \\
\hline $\begin{array}{l}\text { Positive } \\
\text { Small }\end{array}$ & $\begin{array}{lll}0 & 15 & 30\end{array}$ & $\begin{array}{lll}0.3 & 8 & 14\end{array}$ & $\begin{array}{lll}0 & 15 & 30\end{array}$ & $\begin{array}{lll}0 & 15 & 30\end{array}$ & $\begin{array}{lll}0.2 & 0.8 & 1.6\end{array}$ \\
\hline \multirow{2}{*}{$\begin{array}{l}\text { Positive } \\
\text { Medium }\end{array}$} & & & & & \\
\hline & $2660-80$ & $12-1824$ & $2060 \quad 80$ & $\begin{array}{lll}20 & 60 & 80 \\
\end{array}$ & $\begin{array}{lll}1.3 & 2.2 & 2.7 \\
\end{array}$ \\
\hline \multirow{2}{*}{$\begin{array}{l}\text { Positive } \\
\text { Large }\end{array}$} & & & & & \\
\hline & & $20 \quad 30 \quad 45$ & & & $2.4 \quad 3.5 \quad 4.4$ \\
\hline $\begin{array}{l}\text { Positive } \\
\text { Very } \\
\text { Large }\end{array}$ & & & & & $\frac{/}{4.04 .55 .0}$ \\
\hline
\end{tabular}

Figure 15: Linguistic variables and their terms. 
that compounds the difficulty of establishing the membership functions. The reader will notice that we have chosen triangular shaped functions for all our membership functions. That was done simply for the convenience afforded by them. Other possibilities for membership functions include bell-shaped, trapezoidal, etc.

Given a set of linguistic variables and their associated terms, the next question is how to set up a control architecture for the inverted pendulum. Since the rules are expressed using the terms shown in Fig. 15, it is clear that what is needed at the front end of the controller is a process that converts the observations about the state vector into the terms that the rule base can understand. The module that implements this process is called the fuzzification processor. To illustrate an example of fuzzification, suppose the position of link 1 is $+2.5^{\circ}$, meaning link 1 is $2.5^{\prime}$ off from its goal position. From the fuzzy membership functions in Fig. 15, this value of the variable position for link 1 translates into the fuzzy terms positive small and zero with degrees of membership 0.5 and 0.2 , respectively.

Fig. 14 shows the control architecture we have implemented. We have shown two fuzzification processors, one for each link. Each fuzzification processor converts the position and velocity for that link into the relevant terms. The variables $P_{1}, V_{1}$ refer to the position and the velocity values coming from link 1 , the former directly from the encoder attached to link 1 and the latter estimated from consecutive position samples in a straightforward manner. The variables $P_{2}, V_{2}$ shown in the figure are the corresponding quantities for link 2.

As shown in Fig. 14, there is a separate rule base associated with each of the links. Before explaining the nature of the rules, we must hasten to state that the decomposition of the overall rule base into two disjoint sets, each dealing with a separate link, is what makes our architecture different from the previously proposed architectures [39]. From a purely theoretical standpoint, with our decomposition, the inputs to the rules need only be defined in a two-dimensional space, as opposed to the four-dimensional space that would otherwise be called for. For obvious reasons, it would take much more effort to specify a rule if its antecedent spanned all four variables, meaning the two position and the two velocity variables. If the reader would accept the notion that the effort required to specify rules is related exponentially to the number of dependent variables in the antecedents, our scheme, if it can be shown to work, would definitely result in great savings in time and effort.

There is an intuitive rationale for separate processing of the data from the two links. Intuitive arguments similar to what we advanced when we discussed the design of the neural controller also apply here. To repeat briefly, experimental circumstances dictate that link 2 , the vertical link, must have priority over link 1 , the horizontal link, since the former is prone to making the system unstable. Therefore, if the position and the velocity parameters of link 2 are such that an impending instability is indicated. then, a.t least in a simpleminded argument, the motor should simply forget about the needs of link 1 and attend solely to link 2. [For the conditions that indicate whether or not there is an impending instability, the reader is referred to the Section on the neural controller.]

With separate processing of the two links, each branch of the processing in Fig. 14 will have its own 
recommendation for the output motor torque. The next challenge then is to reconcile/combine these recommendations in some useful manner. The considerations that enter into how the two torques reflect the different priorities accorded to the two links. Let $\tau_{1}$ and $\tau_{2}$ be the motor torque recommendations produced by the upper and the lower branches, respectively, shown in Fig. 14. These two torque values are input to the Torque Mediator module shown in Fig. 14. Let the recommendation produced by the Mediator be denoted $\tau$. Then, the resulting torque is,

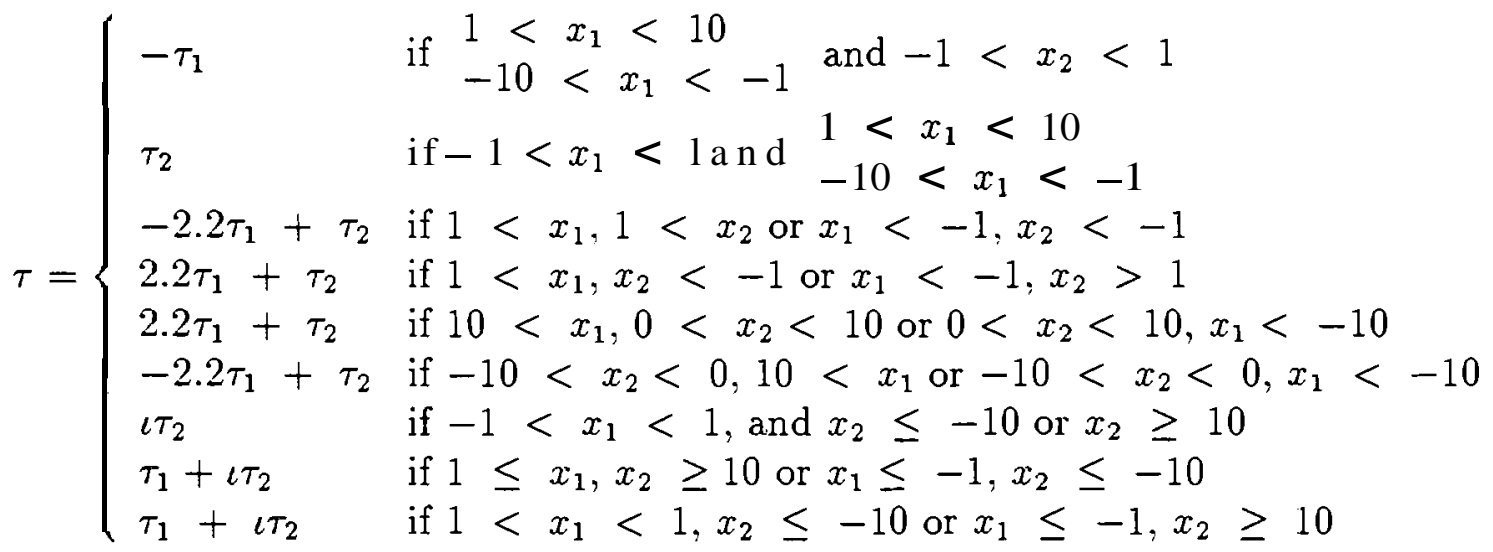

where $x_{1}$ and $x_{2}$ denote the current position of link 1 and link 2 respectively. The constant multiplier $\iota$ receives value 1.25 if one of two position exceeds $|30|^{\circ}$ and 0.87 otherwise. These values are empirically found and reflects that the input torque applied to the motor is adjusted according to the region where the current state position vector resides. The unit for all the numerical values given in Eq. (2.5) is in degrees from the goal state. Recall that the goal state for link two is vertical upright position and a specified position $x_{1}=0$ for link 1 . We have applied an intuitive reasoning, similar to the one used in developing the neural network controller, to combine the two torque values generated by the individual fuzzy controllers. Eq (25) is the explicit representation of that reasoning. For example the very first condition says that if link 1 is close to the goal position and link 1 has not yet reached its goal state, use the torque generated by the fuzzy controller responsible for link 1 as the overall torque value to the motor. Similar statements can be made for all the other conditions.

To complete our presentation of the control architecture of Fig. 14, we will now present more fully those modules that we have discussed only informally so far. Residing in the data/rule base are the fuzzy rules whose antecedents and consequents are fuzzy terms. In general, a multi-input, multi-output fuzzy rule can be expressed in the form of:

$$
I F a_{1} A \ldots \wedge \boldsymbol{a} ; \ldots \wedge a, T H E N c_{1} A \ldots A c_{i} \ldots A \mathrm{c}
$$

where $a_{i}$ 's are the antecedent fuzzy terms and $c_{j}$ 's are the consequent fuzzy terms. For example, two of the fuzzy rules for balancing the inverted pendulum are: 
Tables 1 and 2 show succinctly all the rules currently programmed into the controller, each table applies to a separate branch of the controller in Fig. 14.

Fuzzy inferencing involves the manipulations of the fuzzy membership functions defining the fuzzy sets. Since fuzzy sets are generalizations of crisp, or boolean, sets, their basic set operations are mathematically consistent with that of crisp sets. Fuzzy inferencing strategies are developed using the basic fuzzy set operations $[16,17]$. Among these strategies, the MIN-MAX method is the most popular approach in the existing fuzzy controllers. We will next describe this method.

Associated with each fuzzy rule $R_{k}$, there is a firing strength, $w_{k}$, indicating to what degree the antecedent of the rule is valid. This firing strength is calculated by the basic set operations of the antecedents of each rule. For example, the firing strength of the multi-input, multi-out rule shown above is calculated as:

$$
w_{k}=\min \left(\mu_{a_{1}}(x), \ldots, \mu_{a_{i}}(x), \ldots, \mu_{a_{n}}(x)\right)
$$

where $w_{k}$ is the firing strength associated with rule $R_{k}$, and $\mu_{a_{i}}(x)$ is the degree of membership of input $\mathrm{x}$ with respect to the antecedent fuzzy set $\mathrm{a}$;. Using the intuition that if the antecedent of rule $R_{k}$ is valid to a degree $w_{k}$ then the consequent of rule $R_{k}$ cannot be more valid than $w_{k}$, a clipping process is applied to the consequent of rule $R_{k}$ such that the maximum degree of membership of the consequent subsets are less than or equal to the firing strength $w_{k}$. For example, consequent fuzzy subset $c_{j}^{\prime}$ of rule $R_{k}$ is calculated as:

$$
\mu_{c_{j}^{\prime}}\left(y_{q}\right)=\min \left(w_{k}, \mu_{c_{j}}\left(y_{q}\right)\right)
$$

for every sample point $y_{q}$ in the fuzzy set $c_{j}$. Finally all the consequent elements that share the same domain are combined into a composite fuzzy set using the union operation. In the context of fuzzy controller for the inverted pendulum, all the fuzzy subsets describing torque will be combined into one composite fuzzy set.

The defuzzification processor tries to represent a composite fuzzy subset, by a single numerical value. The most popular defuzzification method is the centroid method where the fuzzy subset is represented by the centroid (i.e. center of mass) of the subset:

$$
y_{0}=\frac{\left(\int \mu_{c_{c} \text { omposite }}\left(y_{q}\right)\left(y_{q}\right) d y\right)}{\left(\int \mu_{c_{c} \text { omposite }}\left(y_{q}\right) d y\right)}
$$

where $y_{0}$ is the defuzzifed value sent to the actuator. 


\begin{tabular}{||c|c|c|c|c|c||}
\hline \hline v1/p1 & NM & NS & ZR & PS & PM \\
\hline NM & PL & PM & PS & PS & ZR \\
\hline NS & PM & PS & PS & ZR & NS \\
\hline ZR & PS & PS & ZR & NS & NS \\
\hline PS & PS & ZR & NS & NS & NM \\
\hline PM & ZR & NS & NS & NM & NL \\
\hline
\end{tabular}

Table 1: Fuzzy Rules for controlling Link 1

\begin{tabular}{||c|c|c|c|c|c|c|c||}
\hline \hline v2/p2 & NL & NM & NS & ZR & PS & PM & PL \\
\hline NM & NVL & NL & NM & NM & NS & PM & PVL \\
\hline NS & NVL & NL & NM & NS & ZR & PM & PVL \\
\hline ZR & NVL & NL & NM & ZR & PM & PM & PVL \\
\hline PS & NVL & NL & ZR & PS & PM & PM & PVL \\
\hline PM & NVL & NL & PS & PM & PM & PM & PVL \\
\hline
\end{tabular}

Table 2: Fuzzy Rules for controlling Link 2

\section{$5 \quad$ Results and Discussion}

We have adopted two criteria to evaluate the performance of the different controllers. The first one is the effectiveness coefficient, which is a ratio of the size of an operational region where the controller is effective to the size of the overall space spanned by the position variables. By effective we mean that, given an initial state position vector, the controller is able to steer the state trajectory successfully such that the goal state is achieved where the goal is specified as the vector $\left[\begin{array}{llll}0 & 0 & 0 & 0\end{array}\right]$. The second performance criterion, the utilization coefficient, is a measure of efficient use of the applied torque with respect to time and the initial position to make the

\begin{tabular}{||l||cc|cc||}
\hline \hline \multicolumn{3}{c}{ simulation } & \multicolumn{2}{c||}{ experiment } \\
\hline link one & 0.606 & $\mathrm{~m}$. & 0.606 & $\mathrm{~m}$. \\
\hline link two & 0.556 & $\mathrm{~m}$. & 0.556 & $\mathrm{~m}$. \\
\hline mass one & 1.927 & $\mathrm{~kg}$. & 1.927 & $\mathrm{~kg}$. \\
\hline mass two & 0.3118 & $\mathrm{~kg}$. & 0.3118 & $\mathrm{~kg}$. \\
\hline sampling time & 0.01 & $\mathrm{~s}$ & $\simeq 0.015$ & $\mathrm{~s}$ \\
\hline gravity & 9.806 & $\mathrm{~m} / \mathrm{s}^{2}$ & 9.806 & $\mathrm{~m} / \mathrm{s}^{2}$ \\
\hline torque range & \pm 5 & $\mathrm{kgm}^{2} / \mathrm{s}^{2}$ & \pm 10 & $\mathrm{v}$. \\
\hline \hline
\end{tabular}

Table 3: The operational parameters. 
system state to transfer from a designated initial state to a goal state. We define the utilization coefficient as

$$
\text { utilization coefficient }=\beta \frac{\sum \tau \kappa}{\boldsymbol{e}}
$$

where $\sum \tau$ is the summed value of the magnitude of the torque over the sampling time to finish a task, $\kappa$ is the normalized sum of the elapsed time for the two links to reach their goal positions, and $\varrho$ is the normalized magnitude of an initial state vector away from the goal. The variable $\beta$ denotes the overall normalization constant so that the utilization coefficient would lie in the interval $[0, \mathrm{I}]$. The normalization is over the same variables obtained from executing the task by all five controllers.

We now show some detailed results of the simulation and the experiment. Various parameter values used for the simulation and the experiment are shown in Table 3. To simulate the dynamic motion of the inverted pendulum using a digital computer, the Euler method was used.

\subsection{PD Controller}

For the PD controller, the constant coefficients $K P_{1}, K P_{2}, K D_{1}$, and $K D_{2}$ are found by manual tuning, and the resulting gain vector $\mathrm{K}$ is

$$
\mathrm{K}=\left[\begin{array}{llll}
2.0 & 12.0 & 0.5 & 2.0
\end{array}\right]
$$

As was mentioned before, this distribution of gain coefficientscauses the second link to be balanced first.

For both simulation and experiment, following steps are taken. First, the second link is removed from its equilibrium point $\mathrm{x}=\left[\begin{array}{llll}0.0 & 0.0 & 0.0 & 0.0\end{array}\right]$ to some initial state vector, which for the simulation results to be discussed is $\mathbf{x}_{\text {initial }}=\left[\begin{array}{llll}0.0 & 20.0 & 0.0 & 0.0\end{array}\right]$, and for the actual experiments $\mathbf{x}_{\text {initial }}=$ $\left[\begin{array}{llll}0.0 & 11.52 & 0.0 & 0.0\end{array}\right]$. Fig. 16 shows the position and velocity trajectories of the simulated system executed by the PD controller along with the input torque values over time. Fig. 17 shows the corresponding experimental result. The solid lines specify the paths of the first link and the dotted lines indicate those of the second link on the position and velocity graphs of Fig. 16 and Fig. 17. The vertical axes are in degrees and degrees/second for the position and velocity graphs, respectively, and the horizontal axis represents the time.

We compute the time interval for the task completion by measuring the elapsed time up to an instant where the position state variables are within a specified region around the goal state and stay in that region afterwards. For simulations, this region around the goal state is easily defined; we set it at $\pm 1^{\circ}$ of the goal position for both links. Note that we can disregard the velocity components for the specification of task completion since for the position variables to remain in the specified region, the velocities have to be near zero. This approach to the characterization of task completion does not really work for the experiments. Friction and other factors cause the trajectory of the state vector to not converge to the predesignated goal state $\mathrm{x}=\left[\begin{array}{llll}0.0 & 0.0 & 0.0 & 0.0\end{array}\right]$ but to some other state that is nearby in the state space and that is consistent with the static 


\begin{tabular}{|c|c|c|c|c|}
\hline & $\overline{\text { initial pos }}$ & total torque & \multicolumn{2}{|c|}{ elapsed time } \\
\hline unit & degrees & $k g-m / s^{2}$ & link 1 & link 2 \\
\hline $\mathrm{PD}$ & {$\left[\begin{array}{lll}0 & 20.0\end{array}\right]$} & 145.65 & $4.4 \mathrm{~s}$. & $3.6 \mathrm{~s}$. \\
\hline $\mathrm{LQ}$ & {$\left[\begin{array}{ll}0 & 20.0\end{array}\right]$} & 85.96 & $14.9 \mathrm{~s}$. & $1.24 \mathrm{~s}$. \\
\hline Neuarl & {$\left[\begin{array}{lll}0 & 20.0\end{array}\right]$} & 105.11 & $2.8 \mathrm{~s}$. & $2.75 \mathrm{~s}$. \\
\hline Nonlinear & {$\left[\begin{array}{lll}0 & 20 & 0\end{array}\right]$} & 259.29 & $4.84 \mathrm{~s}$. & $4.64 \mathrm{~s}$. \\
\hline Fuzzy & {$\left[\begin{array}{lll}0 & 2 & 0.0\end{array}\right]$} & 368.95 & $4.25 \mathrm{~s}$. & $1.13 \mathrm{~s}$. \\
\hline
\end{tabular}

Table 4: Simulation Result

\begin{tabular}{||l||c|c|l|l|}
\hline \hline \multicolumn{1}{c}{ initial pos } & total torque & \multicolumn{2}{c|}{ elapsed time } \\
\hline unit & degrees & volts & link 1 & link 2 \\
\hline PD & {$\left[\begin{array}{ll}0 & 11.52\end{array}\right]$} & 1304 & $0.98 \mathrm{~s}$. & $1.16 \mathrm{~s}$. \\
\hline LQ & {$\left[\begin{array}{ll}0 & 12.6\end{array}\right]$} & 865 & $0.7 \mathrm{~s}$. & $0.87 \mathrm{~s}$. \\
\hline Neuarl & {$\left[\begin{array}{ll}0 & 12.6\end{array}\right]$} & 1338 & $1.13 \mathrm{~s}$. & $1.23 \mathrm{~s}$. \\
\hline Nonlinear & {$\left[\begin{array}{ll}0 & 15.84\end{array}\right]$} & 1148 & $1.15 \mathrm{~s}$. & $1.16 \mathrm{~s}$. \\
\hline Fuzzy & {$\left[\begin{array}{lll}-0.72 & 9.0\end{array}\right]$} & 3692 & $5.24 \mathrm{~s}$. & $5.24 \mathrm{~s}$. \\
\hline \hline
\end{tabular}

Table 5: Experimental Result

friction present in the experiment. It is important to realize that static and dynamic friction, which due to their complexities has not been incorporated in our modeling equation, are functions of the wear and tare of the hardware, the room temperature, humidity, etc. For these reasons, in actual experiments the time interval for task completion is measured up to the point where the two links become stationary.

It is interesting to see that the values of the coefficients, $K P_{1}, K P_{2}, K D_{1}$, and $K D_{2}$, as produced by manual fine tuning, are just what one would expect them to be for the controller to exhibit the correct behavior. To elaborate, suppose the positional deviations in the two links are such that link 2 is leaning away from the goal position in the direction of increasing link 1 deviation. The fact that the gain coefficients of link 'I! are much larger relative to those for link 1 will ensure that link 1 is commanded to turn in a direction so as to bring link 1! into balance even though such an action momentarily increases the deviation of link 1 from its goal position. In other words, the manually obtained values for the coefficients ensure that link 2, being in an unstable state, is accorded a higher priority than link 1 in the reduction of deviations from the goal state. This behavior of the PD controller is confirmed by the experimentally obtained plots in Fig. 17. From the simulation-obtained and experimentally-obtained plots shown in Figs. 16 and 17, we observe that, for the case of simulations, while the first link takes 4.4 seconds to reach the goal position, the first link takes 3.6 seconds. For the experimental case, with the initial state vector given above, the corresponding times are 0.98 and 1.16 seconds, respectively. These results are summarized in 

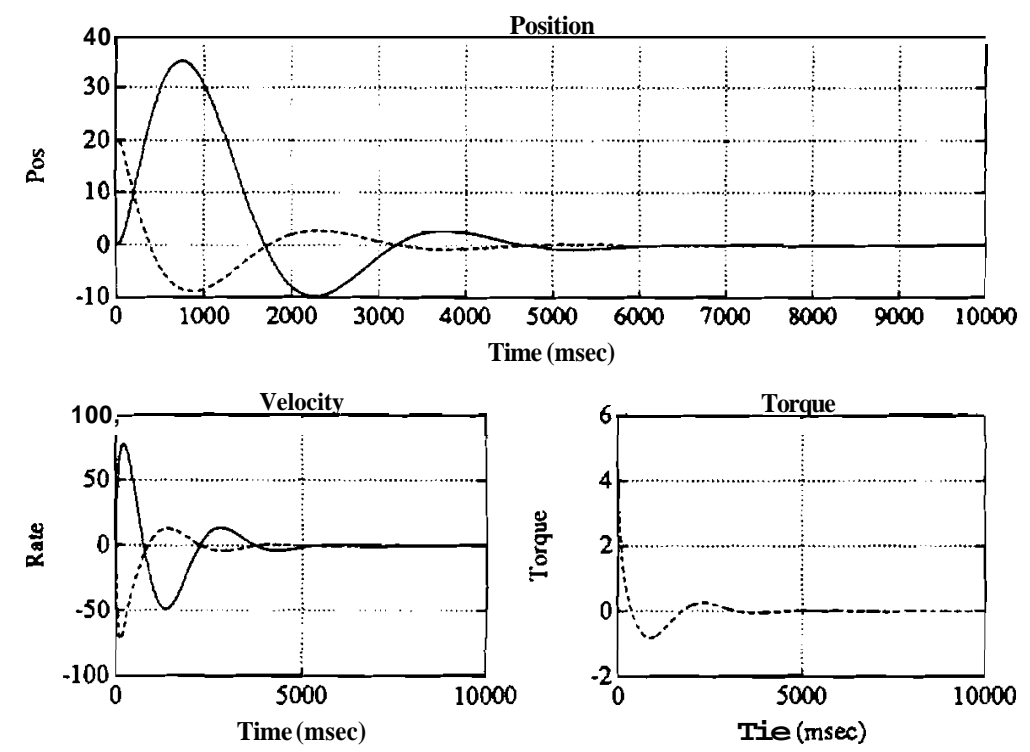

Figure 16: The simulated result using the PD controller

Tables 4 and 5, the former for the case of simulated results and the latter for actual experiments.

For the simulated run shown in Fig. 16, the summation of the absolute value of the torque over time, needed for the system to reach the goal position, is $145.65 \mathrm{kgm}^{2} / \mathrm{s}^{2}$. For the experimental case, since it is not easy to measure the torque directly, following ample precedent we have used the voltage applied to the motor as a measure of the torque produced by the motor. When the magnitude of this voltage is summed at the different sampling times, we obtained 1304 volts for the run shown in Fig. 17. (These results are also displayed in Tables 4 and 5.) We will next discuss issues dealing with the extent of the controllable region for the PD controller.

The controller was tested with various initial positions and we have obtained the following result. In the simulation, the size of the controllable region is defined by the initial deviation of the first link approaching $|142|$ degrees and that of the second link $|47|$ degrees. Now contrast this with the controllable region observed in actual experiments on the inverted pendulum: The controller fails to take the pendulum to its goal position if the deviation on the first link exceeds the interval $\left(-42.0^{\circ}, 38.0^{\circ}\right)$ and $\left(-11.0^{\circ}, 12.0^{\prime}\right)$ for the second link. The lack of symmetry in the positive and the negative deviations, although surprising when first encountered, are explained by the fact that, for both links, the friction is not the same on the two sides of the goal position. The extents of the controllable regions for the various controllers are summarized in Tables 6 and 7 . Evidently, the entries in the tables give us those points where the boundaries of the controllable regions in the subspace spanned by the position variables intersect the position axes. The exact shape of these regions, while difficult to delineate precisely, can be inferred generally by empirical observations, both in simulation and by actual experimentation, in which the inverted pendulum is run with different initial positional deviations for many different cases. The general shape of these regions was found to be elliptical. For inferring this shape, the initial velocities for both links were set to zero in all cases. 


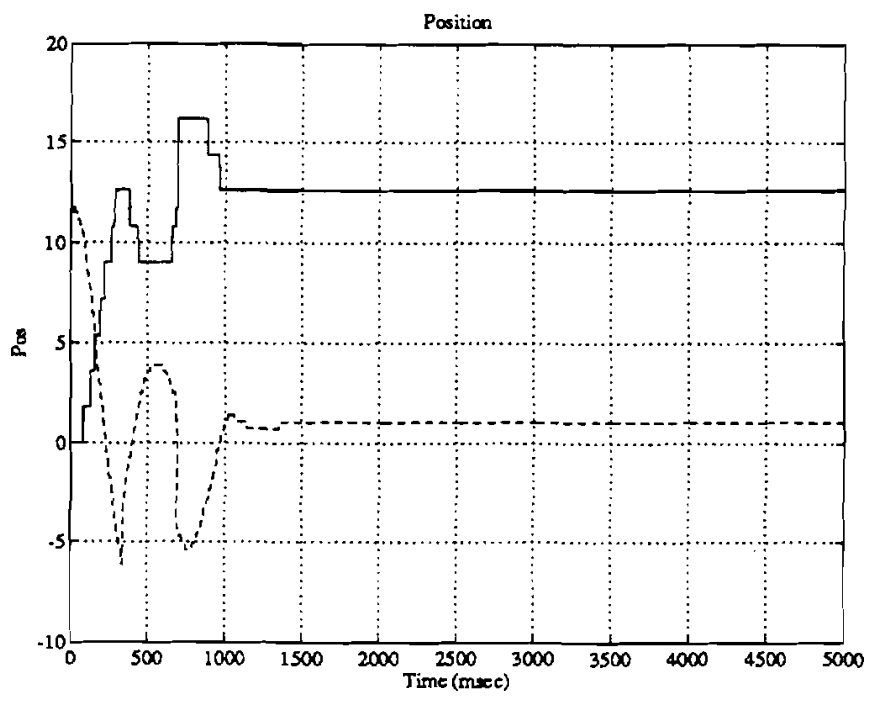

Figure 17: The experimental result using the PD controller

\begin{tabular}{||l|c|c||}
\hline \hline Range( unit degrees) & link one & link two \\
\hline PD Controller & -142 to 142 & -47 to 47 \\
\hline LQ regulator & -180 to 180 & -60 to 60 \\
\hline Neural Controller & -180 to 180 & $-90^{+}$to $90^{-}$ \\
\hline Nonlinear Controller & -160 to 160 & $\mathbf{- 3 2}$ to 32 \\
\hline Fuzzy Controller & -180 to 180 & -40 to 40 \\
\hline \hline
\end{tabular}

Table 6: The simulated result for applicable regions of each controller.

\begin{tabular}{||l|c|c||}
\hline \hline Range(unit degrees) & link one & link two \\
\hline PD Controller & -42 to 38 & -11 to 12 \\
\hline LQ regulator & -152 to 150 & -29 to 35 \\
\hline Neural Controller & -160 to 152 & -35 to 27 \\
\hline Nonlinear Controller & -153 to 146 & -35 to 37 \\
\hline Fuzzy Controller & -168 to 165 & -29 to 36 \\
\hline \hline
\end{tabular}

Table 7: The experimental result for applicable regions of each controller. 
These results show that the PD controller is reasonable only when the second link stays relatively close to its target position. The reason is naturally that the linear mapping of the error values to the torque values is not appropriate for large deviations from the goal state. While the simulation results would have us believe that the PD controller would work even when the deviations are appreciable. the experimental results belie that possibility. While factors, such as friction, may be held accountable for the large discrepancy between what the simulations have to say and what is actually seen in experiment, the reader might wonder whether the simulations have any value at all in this context. Actually, we find the large differences between the simulation and the actual results educational since they are a measure of the extent to which rather intangible factors, such as friction, can influence an experiment.

\subsection{Linear Quadratic Controller}

For the linear quadratic regulator, we have used the following performance index matrix.

$$
Q=\left[\begin{array}{cccc}
0.1 & 0 & 0 & 0 \\
0 & 10 & 0 & 0 \\
0 & 0 & 0.05 & 0 \\
0 & 0 & 0 & 1.0
\end{array}\right]
$$

and $r=1$. The values displayed in the matrix cause the controller to give a higher priority to the position and the velocity of link 2 over the same parameters for link 1. For positional errors, the priority ratio is 100 to 1 , and for velocity errors 20 to 1 . As discussed in Section 4.2.3, the control law is optimized for this given choice of $\mathbf{Q}$ and $\mathbf{r}$. The reader should note that we have not given much attention to analyzing the controller behavior for different choices of $\mathbf{Q}$ and $\mathbf{r}$ and it is entirely possible that an alternative set of values would improve the performance of this controller. However, we believe, any such improvements would be marginal since, after all, we are still dealing with the linearization of a nonlinear control problem and any significant improvements can only be had by not ignoring the nonlinearities.

To find the gain vector that minimizes the performance criterion, we first need to solve the Riccati equation (Eq. 15). To ensure stable solutions, we only want a positive definite solution to the Ricatti equation, and, fortunately, such a solution can be shown to exist and to be unique when a system is controllable [30]. We compute such a solution by using a numerical method given in [27]. The resulting optimal gain vector is

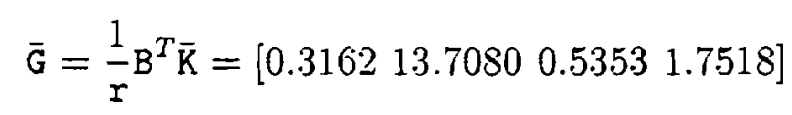

. Fig. 18 shows the position, velocity, and torque trajectories of a simulated system using the linear quadratic regulator with a discrete time observer. Fig. 19 shows the corresponding experimental result.

As was mentioned before, in our system only the position components of the state vector can be measured and the velocity components must be estimated, hence the need for a reduced order 

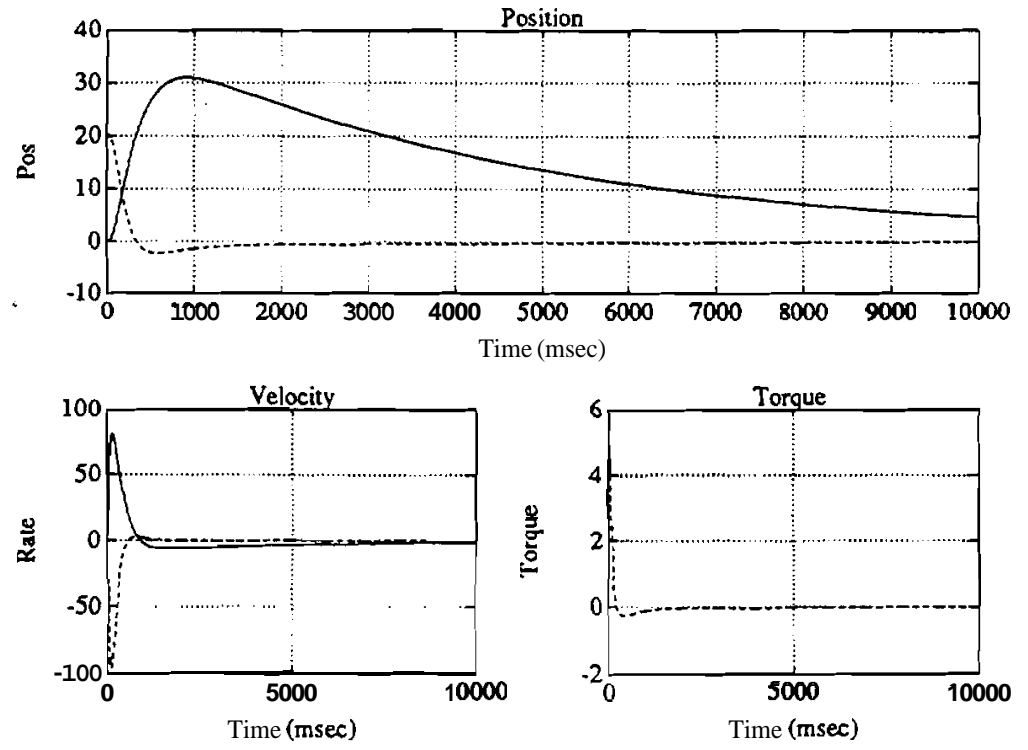

Figure 18: The simulation result using linear quadratic controller

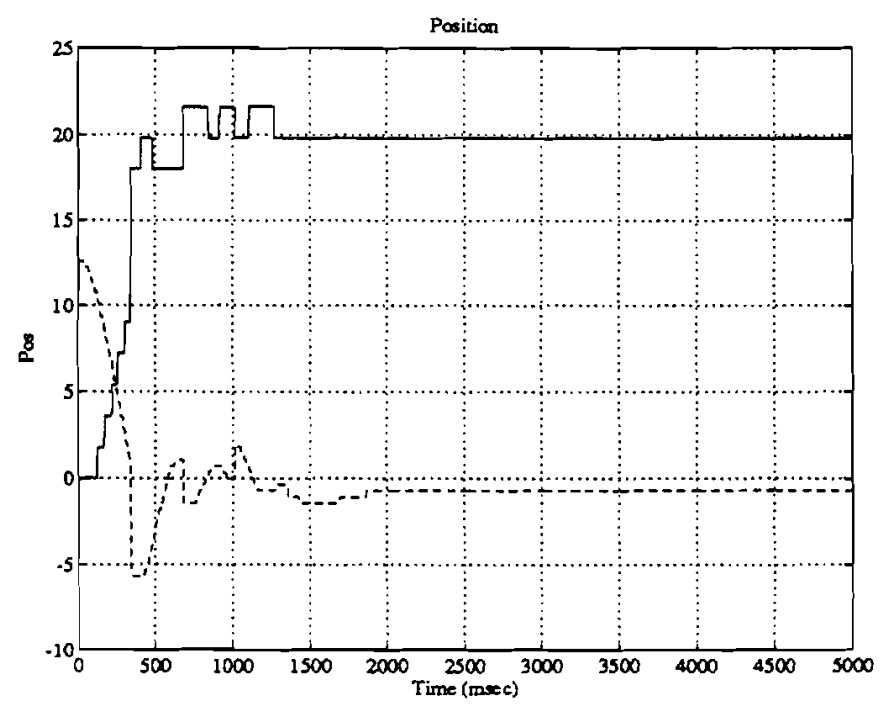

Figure 19: The experimental result using linear quadratic controller 
observer. Our earlier discussion dealt with continuous time. Now we will derive a discrete time version of it; it is this version that is actually used in our experiments.

We start with the linear system(3) and write its equivalent discrete form:

$$
\begin{gathered}
\mathbf{x}(n+1)=\exp (\mathrm{AT}) \mathbf{x}(n)+\left[\exp (\mathrm{AT}) \int_{0}^{\mathrm{T}} \exp (-\mathrm{A} s) \mathrm{B} d s\right] \tau(n)=\mathrm{Mx}(n)+\mathrm{N} \tau(n) \\
\mathrm{y}(n)=\mathrm{C} \mathbf{x}(n)
\end{gathered}
$$

We introduce another internal vector state variable $w$ and set

$$
\mathrm{w}(n+1)=\mathrm{P} w(n)+\mathrm{L} \tau(n)+\mathrm{Vy}(n)
$$

where $\mathrm{P}, \mathrm{L}$, and $\mathrm{V}$ are unknown matrices to be computed. We desire that the w state approach some combination of the system state $\mathrm{x}$, i.e., $\mathrm{w}(n)=\mathrm{Sx}(n)$ as $\mathrm{n}$ becomes large. We choose $\mathrm{S}$ as

$$
S=\left[\begin{array}{llll}
s_{11} & s_{12} & 1 & 0 \\
s_{21} & s_{22} & 0 & 1
\end{array}\right]
$$

These entries in the S matrix allow us to express the velocity components as linear combinations of the positional components and the state vector $\mathrm{w}$.

The difference between w and $\mathbf{S x}$, which we wish to approach zero, is

$$
\mathrm{w}(n+1)-\mathrm{Sx}(n+1)=\mathrm{Pw}(n)+(\mathrm{VC}-\mathrm{SM}) \mathbf{x}(n)+(\mathrm{L}-\mathrm{SN}) \tau(n)
$$

We desire $(\mathbf{w}-\mathbf{S} \mathbf{x})(n+1)=\mathrm{P}(\mathbf{w}-\mathrm{Sx})(n)$. This equality indicates that the components of $\mathrm{P}$, if assigned appropriate values, control the rate of convergence of the error to zero. Comparing this equality with Eq. (34), we get VC - SM $=$ PS and L $=$ SN. Since we desire a deadbeat ${ }^{7}$ performance [3], we choose $P$ to be zero and by equating $V C=S M$

$$
\left[\begin{array}{ll}
v_{11} & v_{12} \\
v_{21} & v_{22}
\end{array}\right]\left[\begin{array}{llll}
1 & 0 & 0 & 0 \\
0 & 1 & 0 & 0
\end{array}\right]=S M
$$

and computing $\mathrm{M}$ and $\mathrm{N}$ matrices numerically [28] with the sampling time 0.01 second, we found the components of the S matrix $\left\{s_{11}, s_{12}, s_{21}, s_{22}\right\}=\{-100,0.01,0,-100.29\}$, the components of the V matrix, $\left\{v_{11}, v_{12}, v_{21}, v_{22}\right\}=\{-100,-0.062,0,-99.99871$, and

$$
L=\left[\begin{array}{c}
0.0282 \\
-0.0615
\end{array}\right]
$$

Thus,

$$
\mathrm{w}(n+1)=\mathrm{L} \tau(n)+\mathrm{V}\left[\begin{array}{l}
x_{1} \\
x_{2}
\end{array}\right]
$$

\footnotetext{
'In the continuous time case, we had a freedom to choose the locations of the poles in the s-plane such that the error between the estimating state vector and the true state vector decayed exponentially. In the discrete time case, we can do much better than the exponential decay by setting the poles in z-plane to be identically zero. The result is that the error between the estimated state and the real system state settles exactly down to zero after at most a number of samples equaling the order of the system. Such a control scheme is known as deadbeat control.
} 
and from Eq. (33), we can solve for the estimates

$$
x_{3}(n+1)=w_{1}(n+1)+100 x_{1}(n+1)-0.01 x_{2}(n+1)
$$

and

$$
x_{4}(n+1)=w_{2}(n+1)+100.29 x_{2}(n+1)
$$

From Fig. 18, which is a result of simulation, with the initial state vector given in Table 4, we see that it takes over 10 seconds for link 1 to arrive at its goal position while for link 2 it only takes about a second. The summed torque over the duration of the control is $83.179 \mathrm{kgm}^{2} / \mathrm{s}^{2}$. It seems that the time for link 1 to reach its target position is much too long. That is, however, due to the performance index we have chosen. Observe that in the quadratic performance index $Q$ we did not put too much emphasis on the first link and its velocity, and the performance result reflects just that. Also, if we had much smaller index $\mathbf{r}$, the time it took for the first link to reach its target would have been much quicker; but then, we would have to pay for it with a larger value for the total torque. The controllable region was found to be $-\pi$ to $\pi$ for the link 1 and $-\pi / 3$ to $\pi / 3$ for link 2 . Table 4 shows the controllable space of the simulated system using the linear quadratic regulator.

Fig. 19 shows the experimental result with the initial state described in Table 5. For task completion, the elapsed times are 0.7 and 0.87 seconds for link 1 and link 2 , respectively. The relatively short interval for link 1 compared to what it was for case of simulation is again due to the static friction of link 1 . If friction did not exist, link 1 would have moved slowly toward the final goal position. Table 7 shows the corresponding controllable region obtained via the experiment. Before closing our discussion on the linear quadratic controller, we'd like to mention that, despite the more complex arguments that go into its derivation, a linear quadratic controller is no different from a PD controller at the implementation level. In other words, in Fig. 8, the gain matrix $G$ has the same structure as the gain matrix for a. PD controller.

\subsection{Neural Network Controller}

Table 8 shows the various parameter values used for the neural controller described in section

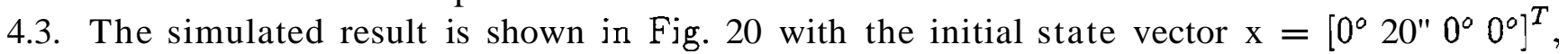
and the corresponding experimental result is shown in Fig. 21 with the initial state vector $\mathrm{x}=$ $\left[\begin{array}{lll}0^{\circ} & 12.6 " 0^{\prime \prime} & 0^{\circ}\end{array}\right]^{T}$. The elapsed time intervals for the task completion of the simulated sytem are 2.8 and 2.75 seconds for link 1 and link 2, respectively. For the experimental case, the time intervals for link 1 and link 2 are 1.13 and 1.23 seconds. The total summed torque value required for the task is $105.11 \mathrm{kgm}^{2} / \mathrm{s}^{2}$ for the simulated system and 1338 volts for the experimental case. In the simulation case, the extreme points for the controllable region are found to be $-\pi$ and $\pi$ for link 1 and $-\pi / 2^{+}, \pi / 2^{-}$for link two. The corresponding extreme points for the experiment are $-160^{\circ}$ and $152^{\circ}$ for link 1 and $-35^{\prime \prime}$ and $27^{\circ}$ for link 2 . 

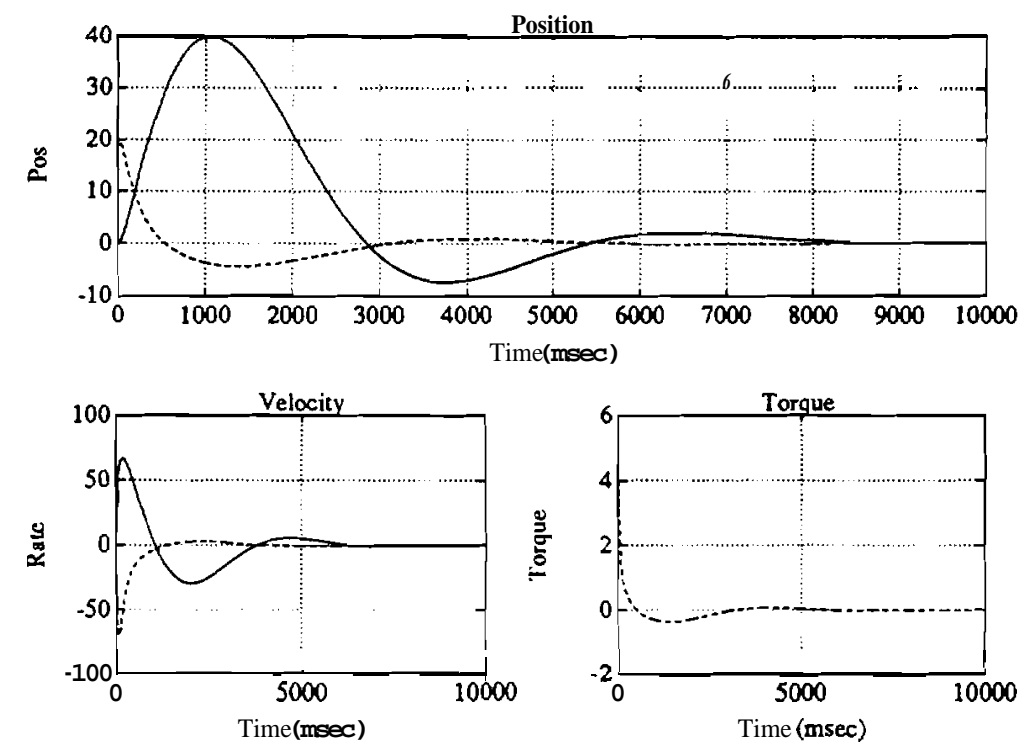

Figure 20: The simulated result using the neural controller

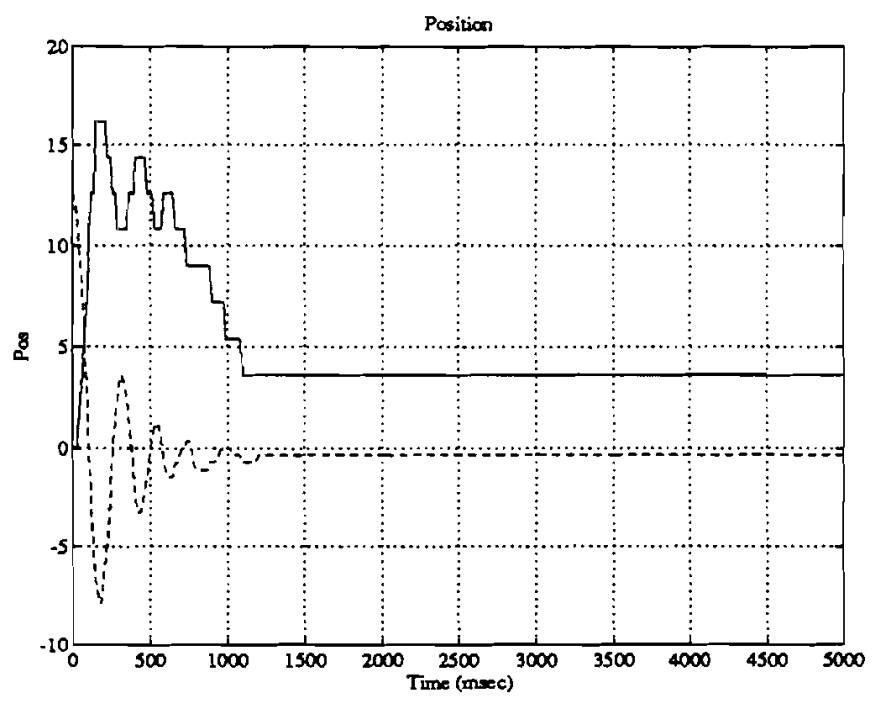

Figure 21: The experimental result using the neural controller 
pack/meng/kak

\begin{tabular}{||l||l||}
\hline \hline input layer units & 4 \\
\hline first hidden layer units & 8 \\
\hline second hidden layer units & 24 \\
\hline number of training loop & 1000 \\
\hline number of iteration & 300 \\
\hline number of individual data learning & 5 \\
\hline limit of convergence error & 0.005 \\
\hline initial weight range & \pm 1 \\
\hline sigmoid output range & 0 to 1 \\
\hline learning rate & 1.0 \\
\hline learning momentum & 0.4 \\
\hline learning second momentum & -0.2 \\
\hline constant K1 & 1.0 \\
\hline constant K2 & 1.0 \\
\hline constant Kd1 & 0.1 \\
\hline constant Kd2 & 1.0 \\
\hline constant Kp1 & 1.0 \\
\hline constant Kp2 & 1.0 \\
\hline \hline
\end{tabular}

Table 8: The parameter values for the neural controller. 


\subsection{Nonlinear Controller}

For the nonlinear controller, the coordinate transformation described in section 4.4.2 is performed first. To compute the various Lie derivatives needed for the transformation, defined in section 4.4.1, and the inverse of the nonlinear controllability matrix $\mathrm{C}$, the symbolic software Mathematica [36] is used. Since, the original dynamics for the inverted pendulum requires computation of a large number of derivatives and Jacobian matrices, we simplified the dynamics such that it contains only the main nonlinear terms, the inertia terms and the gravity term; that is, we approximated the dynamics of the system to simplify the controller construction.

Using the approximated model, the last row of the inverse of the nonlinear controllability matrix $\mathcal{C}$ is found to be $\mathrm{q}=\left[-\operatorname{asec} x_{2}-\mathrm{bsec} x_{2} 00\right]$ where $\mathrm{a}=0.01$ and $\mathrm{b}=0.0046$. In general, the partial differentialequation in Eq. (24) can not be solved analytically. However, as was first pointed out in [33] and [41], if we instead solve the differential equation

$$
\frac{\partial \mathrm{T}_{1}}{\partial \mathrm{x}}=\left[\begin{array}{lllll}
0 & 0 & \ldots & 0 & \mu
\end{array}\right] \mathcal{C}^{-1}
$$

for any choice of $\mu$ that makes the differential equation solvable, it follows straightforwardly from the arguments in Appendix $\mathrm{C}$ that the resulting dynamic equation for $z$ will be

$$
\dot{\mathbf{z}}=\left[\begin{array}{c}
z_{2} \\
z_{3} \\
\vdots \\
z_{n} \\
\hat{\mathbf{w}}\left(z_{1}, z_{2}, \ldots, z_{n}\right)
\end{array}\right]+\left[\begin{array}{c}
0 \\
0 \\
\vdots \\
0 \\
\mu(\mathbf{z})
\end{array}\right] \tau
$$

Now, as far as the implementation is concerned, there really is not much difference between the dynamic equation Eq. (23) and the dynamic equation Eq. (36). The difference that does exist is in the size of the input torque.

We will choose $\mu=-\cos x_{2}$ as this will transform the partial differential equation Eq. (24) into

$$
\begin{aligned}
\frac{\partial \mathrm{T}_{1}}{\partial \mathrm{x}} & =\left[\begin{array}{lllll}
0 & 0 & \ldots & 0 & \mu
\end{array}\right] \mathcal{C}^{-1} \\
& =\left[\begin{array}{llll}
a & b & 0 & 0
\end{array}\right]
\end{aligned}
$$

The solution of this partial differential equation is $z_{1}=T_{1}(\mathrm{x})=a x_{1}+b x_{2}$.

We compute the rest of the components of the transformation by applying the Lie derivative of the second type iteratively.

$$
\begin{aligned}
z_{2}=\mathrm{L}_{f} \mathrm{~T}_{1} & =\frac{\partial \mathrm{T}_{1}}{\partial \mathrm{x}} \mathrm{f} \\
& =[\mathrm{a}, \mathrm{b}, 0,0] \mathrm{f} \\
& =a x_{3}+b x_{4}
\end{aligned}
$$




$$
\begin{aligned}
z_{3}=\mathrm{L}_{\mathrm{f}}^{2} \mathrm{~T}_{1} & =\frac{\partial\left(\mathrm{L}_{\mathrm{f}} \mathrm{T}_{1}\right)}{\partial \mathrm{x}} \mathrm{f} \\
& =[0,0, a, b] \mathrm{f} \\
& =a c \sin x_{2}+\text { bd } \sin x_{2}
\end{aligned}
$$

where $c=-5.2344$ and $d=29.0468$. And

$$
\begin{aligned}
z_{4}=\mathrm{L}_{f}^{3} \mathrm{~T}_{1} & =\frac{\partial\left(\mathrm{L}_{f}^{2} \mathrm{~T}_{1}\right)}{\partial \mathrm{x}} \mathbf{f} \\
& =\left[0,(a c+b d) \cos x_{2}, 0,0\right] \mathrm{f} \\
& =(a c+b d) x_{4} \cos x_{2} v v
\end{aligned}
$$

The resulting transformation from the $\mathrm{x}$ space to the $z$ space is

$$
\left[\begin{array}{c}
z_{1} \\
z_{2} \\
z_{3} \\
z_{4}
\end{array}\right]=\left[\begin{array}{c}
a x_{1}+b x_{2} \\
a x_{3}+b x_{4} \\
(a c+b d) \sin x_{2} \\
(a c+b d) x_{4} \cos x_{2}
\end{array}\right]=\left[\begin{array}{c}
\mathrm{T}_{1} \\
\mathrm{~L}_{f} \mathrm{~T}_{1} \\
\mathrm{~L}_{f}^{2} \mathrm{~T}_{1} \\
\mathrm{~L}_{\mathrm{f}}^{3} \mathrm{~T}_{1}
\end{array}\right]
$$

Differentiating the transformation matrix $T$ with respect to $\mathrm{x}$ and substituting variable $\mathrm{x}$ with its equivalent form in the new coordinate frame in $z$ produces

$$
\dot{\mathbf{z}}=\left[\begin{array}{c}
z_{1} \\
z_{2} \\
\tilde{z}_{3} \\
\hat{\mathbf{w}}(\mathbf{z})
\end{array}\right]+\left[\begin{array}{c}
0 \\
0 \\
0 \\
(a c+b d) r \cos \left[\sin ^{-1}\left(\frac{z_{3}}{a c+b d}\right)\right]
\end{array}\right] \tau
$$

where

$\mathbf{r}=-13.3163$, and

$$
\hat{\mathbf{w}}(\mathbf{z})=-z_{3} \mathrm{H}^{2}+d z_{3} \cos \left[\sin ^{-1}\left(\frac{z_{3}}{a c+b d}\right)\right]
$$

$$
\mathrm{H}=\frac{54}{(o c+b d) \cos \left(\sin ^{-1}\left(\frac{z_{3}}{a c+b d}\right)\right)}
$$

Note that the value of the term $(a c+b d) r$ in Eq. (39) is -1 as it should.

Then, in accordance with the discussion in Section 4.4.2, the control input needed for canceling the nonlinear part of the dynamic equation and for the control of the subsequently linearized equation is:

$$
\begin{aligned}
\tau(\mathrm{z})= & \frac{1}{\mathrm{R}}\left\{z_{3} \mathrm{H}^{2}-d z_{3} \cos \left(\sin ^{-1}\left(\frac{z_{3}}{a c+b d}\right)\right)\right. \\
& \left.-\eta_{0} z_{1}-\eta_{1} z_{2}-\eta_{2} z_{3}-\eta_{3} z_{4}\right\}
\end{aligned}
$$

where

$$
\mathrm{R}=(a c+b d) r \cos \left[\sin ^{-1}\left(\frac{z_{3}}{a c+b d}\right)\right]
$$



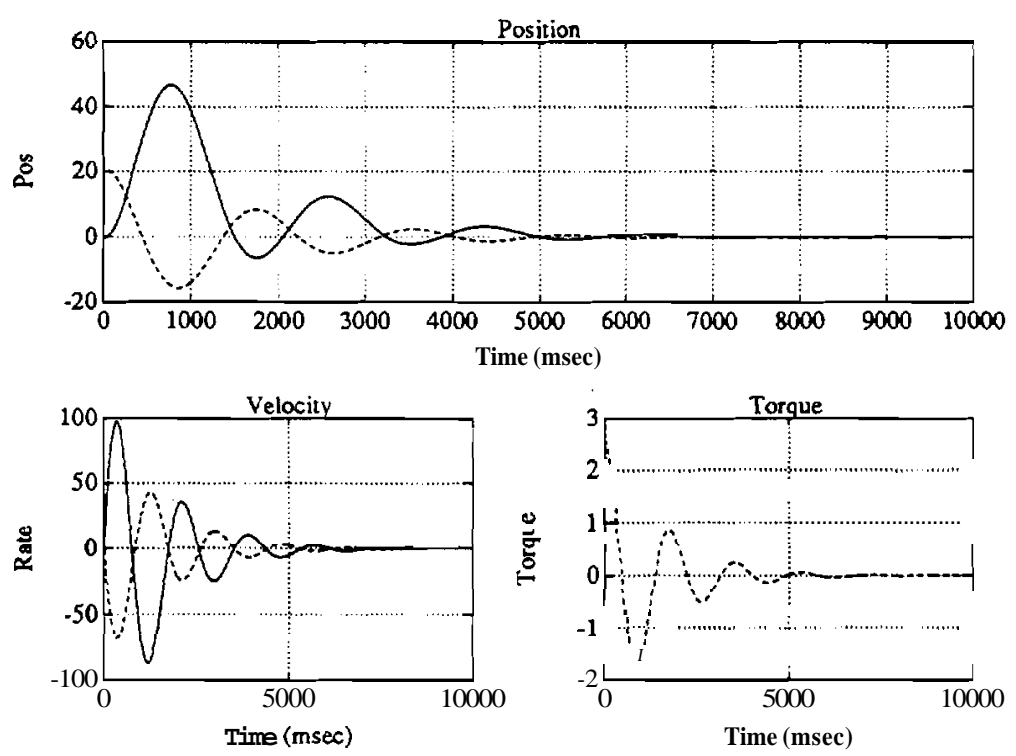

Figure 32: The simulated result using the nonlinear controller

The coefficients $\eta_{0}, \eta_{1}, \eta_{2}$, and $\eta_{3}$ can be set in the same manner as for a linear control scheme but, of course, in the $z$ space. We first tried the linear quadratic regulator in the $z$ space using the approximate relationships between the the variables $\mathrm{x}$ and $\mathbf{z}$. This did not generate desired results; therefore, we switched to the pole assignment method, which is valid since any diffeomorphic transformation preserves the eigenvalues of the original system about an operating point [13].

The eigenvalues of the plant matrix associated with the resulting linear feedback (see Appendix A) are selected to be $\mathrm{e}=\{-.5,-5,-1 \pm 1.33 i\}$; the corresponding coefficients in Eq. (40) are then given by $\eta_{0}=69.225, \eta_{1}=77.689, \eta_{2}=47.769$, and $\eta_{3}=12.0$.

Even though the above control law was derived in the $z$ space, actual control will still need to be effected in the $x$ space. The control input in the $x$ space is obtained by substituting for the $z$ components from Eq. (38)

$$
\begin{aligned}
\tau(\mathbf{x}) & =\frac{1}{(\mathrm{ac}+b d) r \cos x_{2}} *\left[(\mathrm{ac}+b d) \sin x_{2} x_{4}^{-2}\right. \\
& -d(a c+b d) \sin x_{2} \cos x_{2}-\eta_{0}\left(a x_{1}+\mathrm{b} x_{2}\right) \\
& -\eta_{1}\left(a x_{3}+\mathrm{b} x_{4}\right)-\eta_{2}(a c+b d) \sin x_{2} \\
& \left.-\eta_{3}(a c+b d) x_{4} \cos x_{2}\right]
\end{aligned}
$$

Figs. 22 and 23 show the simulated and experimental results when the torque computed by using the above formula is applied to the system. From Fig. 22, it takes 4.84 and 4.64 seconds for link 1 and link 2, respectively, to reach their designated goal positions. The corresponding results for the experimental case are 1.15 and 1.16 seconds for link 1 and link 2, respectively. The total summed torque needed to steer the state variable to its goal state is $259.29 \mathrm{kgm}^{2} / \mathrm{s}^{2}$ for the simulation 
and 1148 volts for an actual experiment. The controllable region for the simulated case is $\left(-160^{\circ}\right.$, $\left.160^{\circ}\right)$ for link 1 and $\left(-32^{\circ}, 32^{\prime \prime}\right)$ for link 2 . For the experimental case, the region spans $\left(-153^{\circ}\right.$, $\left.146^{\prime \prime}\right)$ for link 1 and $\left(-25^{\circ}, 27^{\prime \prime}\right)$ for link 2.

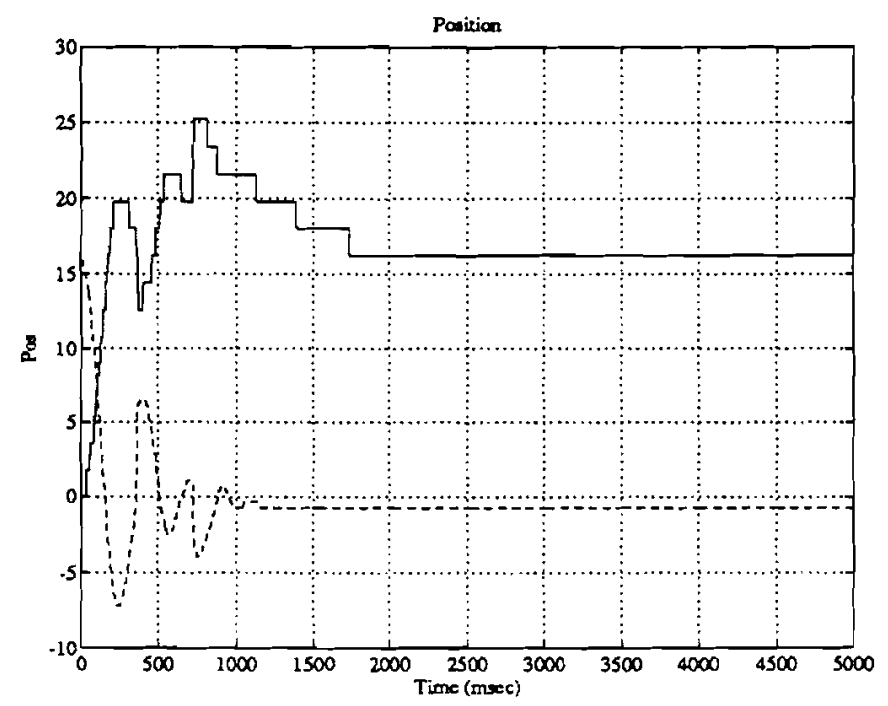

Figure 23: The experimental result using the nonlinear controller

\subsection{Fuzzy Controller}

Fig. 24 shows the simulated result when fuzzy logic based controller is used with an initial state vector $\mathbf{x}=\left[\begin{array}{llll}0.0 & 20.0 & 0.0 & 0.01\end{array}\right.$. The elapsed time for the first link to arrive at its goal position is 4.25 seconds while it takes only 1.13 seconds for the second link to reach its goal position. The required torque value for completion of the task is $368.95 \mathrm{~kg}-\frac{\mathrm{m}}{\mathrm{s}^{2}}$. Fig. 25 shows the corresponding experimental result. The time interval for the link one and link two to achieve the goal positions are both over 5 seconds. Both link one and two do not come to rest and continue to oscillate around the goal state. This can be attributed to the nature of a fuzzy logic based controller. That is unless we have a large number of dense fuzzy membership functions for both input and output around the goal state and the desired output, we will always experience this oscillating effect. Issues pertaining to the minimization of such oscillations are outside the scope of this paper. The measured voltage value is 3692 volts. 

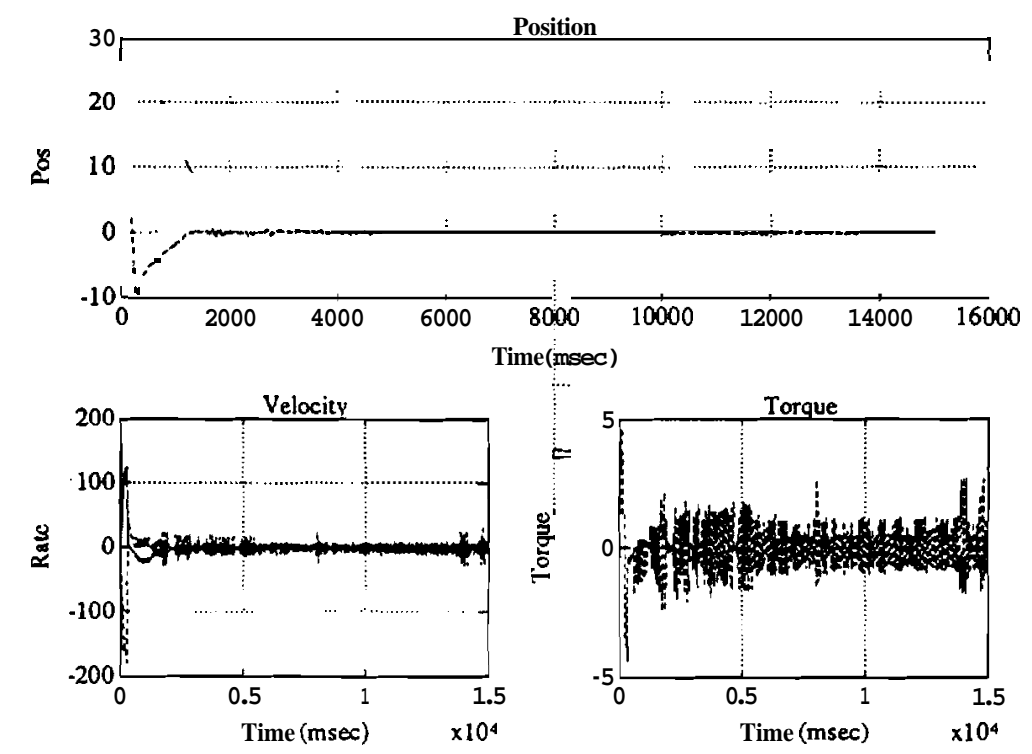

Figure 24: The simulated result using the fuzzy controller

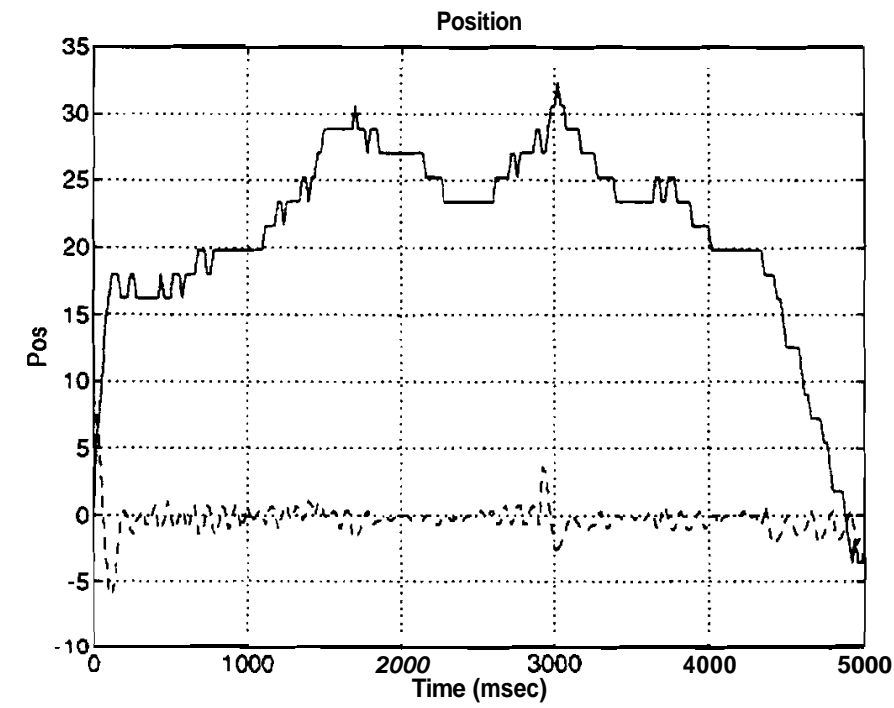

Figure 25: The experimental result using the fuzzy controller 


\begin{tabular}{|l|c|c||}
\hline \hline \multicolumn{1}{|c}{} & \multicolumn{1}{c}{ Simulation } & \multicolumn{1}{c|}{ Experiment } \\
\hline PD Controller & 0.324 & 0.022 \\
\hline Linear Quadratic Controller & 0.524 & 0.234 \\
\hline Neural Network Controller & 0.785 & 0.272 \\
\hline Non-linear Controller & 0.248 & 0.203 \\
\hline Fuzzy Logic Controller & 0.349 & 0.262 \\
\hline \hline
\end{tabular}

Table 9: The effectiveness coefficient

\begin{tabular}{||l|c|c||}
\hline \hline \multicolumn{1}{ll}{} & Simulation & Experiment \\
\hline PD Controller: & 0.473 & 0.230 \\
\hline Linear Quadratic Controller: & 0.563 & 0.123 \\
\hline Neural Network Controller: & 0.237 & 0.170 \\
\hline Non-linear Controller: & 1.0 & 0.179 \\
\hline Fuzzy Logic Controller: & 0.804 & 1.0 \\
\hline \hline
\end{tabular}

Table 10: The utilization coefficient

\section{Conclusion}

We summarize the overall results with the help of Tables 9 and 10. The PD controller, the neural network controller, and the fuzzy logic controller fall into the category of controlling the system without any specific system model, whereas the linear quadratic controller and the nonlinear controller require an accurate model of the system dynamics to compute suitable control commands to the motor. For many cases, a detailed and accurate dynamics model of a system is difficult to specify. In fact, it is almost impossible to come up with an exact mathematical model for any system. By constructing a mathematical model, the designer can only hope to emulate the more prominent characteristics of the actual system. Given the difficulties in specifying an exact model, one may choose to use non-model based approaches, such as a PD controller, a neural controller, or a fuzzy logic based controller. For a detailed analysis of a system, however, a model based controller is often necessary. In the case of the inverted pendulum, the model offers an understanding of how various components of the dynamic model affect the overall performance.

The effectiveness coefficient is a useful criterion on the basis of which a controller can be selected for a particular task. For the inverted pendulum, the PD controller is applicable only near the equilibrium point as shown in the Tables 6 and 7 (also see Table 9 for comparison). Experimental results confirm that the neural controller has a larger controllable region compared to the other controllers. Note that the linear quadratic controller has also a large controllable region. In the simulation study, this region turned out to be even larger than the regions for the nonliner controller and the fuzzy logic controller. In the experiments, however, the controllable region for 
the linear quadratic controller is larger than that for only the nonlinear controller. The reason can be attributed to the non-modeled dynamic factors that are part and parcel of real experiments. The fuzzy logic based controller was much more adaptive to these nonlinear effects than the linear quadratic controller. The smaller controllable region for the nonlinear controller is due to the fact that we made an approximation of the inverted pendulum dynamics in constructing the controller. Empirical results show that when the second link approaches \pm 40 degrees angle from the vertical, the system becomes unstable and uncontrollable for any controller. The large torque generated at those instances are not countered well by the subsequent input torques.

The utilization coefficient gives another useful index for comparing a controller with the others. As can be seen from the Table 10, the fuzzy logic based controller and the nonlinear controller are wastful in their torque consumption. The small values for the linear quadratic controller reflects the efficiency of a linear controller when operated near the equilibrium point. We have known that a linear quadratic controller is quite suitable for tasks near equilibrium points. This shows that linear quadratic controller, in addition to its effectiveness when system states do not deviate far away from an equilibrium point, is also efficient in its consumption of control inputs. A designer, however, should consider other factors such as a size of the desired controllable region in selecting a controller for tasks that require a large control area around an operating point.

In closing, we summarize the deficiencies for each controller briefly. The PD controller performs well near the equilibrium point but has too limited a controllable region. The linear quadratic controller and nonlinear controllers are prone to modeling errors. Also, the derivation of the nonlinear controller using the full dynamics of the system is impractical due to numerous vector derivatives and Jacobian matrices that need to be computed. Also, it is not clear how one would systematize the generation of training patterns for the neural controller. With regard to the fuzzy logic controller, selecting and tuning the membership functions and rules are cumbersome.

In summary, we considered five different types of controllers and their performance for controlling a two-link inverted pendulum, a nonlinear problem. We studied the performance of each controller using two criteria: effectiveness coefficient which is the ratio of the area of the controllable region to the area of the entire space spanned by the position variables associated with the two links; and utilization coefficient which is a measure of the economy of the control input required for the inverted pendulum to transition for an initial state to the goal state. By introducing these two new criteria, we can now evaluate the performance of any controller vis a vis other controllers.

The overall conclusion of the present study is that, on the basis of the effectiveness coefficient comparison, the neural network based controller gives rise to the largest controllable region in the space spanned by the position variables. We also showed that if the pendulum is constrained to operate in close vicinity of the equilibrium state, the linear controllers give the most efficient performance with respect to the utilization coefficient. Among the nonlinear controllers, the neural network controller gives the best performance with respect to the utilization coefficient. 
Acknowledgement

The authors would like to thank Stanislaw Zak for giving us valuable pointers in the literature on nonlinear control methods. Also, Ken Groom taught us how to use a transputer based system for control.

\section{References}

[1] C.A. Anderson, "Strategy Learning with Multilayer Connectionist Representations." In Proceedings of the Fourth International Workshop on Machine Learning. Irvine, CA, 1988.

[2] Barto et al., "Neuronlike Adaptive Elements that can Solve Difficult Learning Control Problems." In IEEE Transactions on Systems, Man. and Cybernetics. vol. smc-13, pp. 834-846, 1983.

[3] J. Billingsley Controlling with computers. McGraw-Hill, 1989.

[4] W. M. Boothby, An Introduction to Differentiable Manifolds and Riemannian Geometry. Academic Press, San Diego, 1986.

[5] R.W. Brockett, "System theory on group manifolds and coset spaces." In SIAM J. Control. vol. 10, pp. 265-285, 1972.

[6] R.W. Brockett, "Robotic Manipulators and the product of exponentials formula." In Proceedings of the MTNS-83 International Symposium. pp. 120-129, 1983.

[7] G. Cybenko, "Approximation by Superpositions of a Sigmoidal Function." In Mathematics of Control, Signals: and Systems. vol. 2, pp. 303-314, 1989.

[8] R.A. Decarlo, Linear Systems Prentice-Hall Inc, 1989.

[9] K. Funahashi, "On the Approximate Realization of Continuous Mappings by Neural Networks." In Neural Networks. vol. 2 pp. 183-192, 1989.

[10] S. Ganguly, T.J. Tarn and A.K. Bejczy, "Control of Robots with Discrete Nonlinear Model: Theory and Experimentation." In 1991 IEEE International Conference on Robotics and Automation. pp. 528-533, Sacramento, April 1991.

[11] Golub and V. Loan, Matrix Computations., Johns Hopkins, 1990.

[12] J.J. Helferty, J.B. Collins and M. Kam, "A Neural Network Learning Strategy for the Control of a One-legged Hopping Machine." In 1989 IEEE International Conference on Robotics and Automation. pp. 1604-1609, Scottsdale, May 1989. 
[13] L.R. Hunt, R. Su and G. Meyer, "Design for Multi-input Nonlinear Systems" In R.W. Brockett, R.S. Millman and H.J. Sussman,(Eds), Diflerential Geometric Control Theory. Birkhauser, Boston, vol. 27, pp.268-298, 1983.

[14] V. Jurdjevic and H.J. Sussmann, "Control Systems on Lie Groups." In Journal of Diflerential Equations. vol. 12, pp. 313-329, 1972.

[15] J.G. Kuschewski, S. Hui and S.H. Zak, "Application of Feedforward Neural Networks to Dynamical System Identification and Control." In IEEE Transactions on Control Systems Technology vol. 1, no. 1, pp. 37-49, 1993.

[16] C. C. Lee, "Fuzzy Logic in Control Systems:Fuzzy Logic Controller - Part I," In IEEE Transactions on Systems, Man, and Cybernetics. Vol. 20, No. 2, pp. 404-418, March/April, 1990.

[17] C. C. Lee, "Fuzzy Logic in Control Systems:Fuzzy Logic Controller - Part II," In IEEE Transactions on Systems, Man. and Cybernetics. Vol. 20. No. 2, pp. 419-435. March/April, 1990.

[18] D.G. Luengerger, "Observing the State of a Linear System." In IEEE Trans. on Military Electronics. vol. MIL-8, pp. 74-80, April 1964.

[19] E. H. Mamdani and S. Assilian, "An Experiment in Linguistic Synthesis with a Fuzzy Logic Controller." In International Journal of Man-Machine Studies. vol. 7, No. 1, pp. 1-13, 1975.

[20] M. Meng and A.C. Kak, "Fast Vision-Guided Mobile robot Navigation Using Neural Networks." In IEEE International Conference on System, Man, and Cybernetics. pp. 111116Chicago, IL, 1992.

[21] H. Miyamoto et al., "Feedback-Error-Learning Neural Network for Trajectory Control of a Robotic Manipulator.", In Neural Networks. vol. 1, pp. 251-265, 1988.

[22] S. Nagata, M. Sekiguchi and K. Asakawa, "Mobile robot Control by a Structured Hierarchical Neural Network." In IEEE Control System Magazine. vol. 10, No. 3, pp. 69-76, 1990.

[23] H. Nijmeijer and A.J. Van der Schaft, Nonlinear Dynamical Control Systems. Springer-Verlag, New York, 1990.

[24] D.J. Pack, M. Meng and A.C. Kak, "Comparative Study of Motion Control Methods for a Nonlinear System." School of Electrical Engineering, Purdue University, W. Lafayette, Indiana, Robot Vision Laboratory, Technical Report TR-EE 92-41 1992.

[25] B. Paden and S. Sastry, "Optimal Kinematic Design of 6R Manipulators." In The International journal of Robotics Research. vol. 7, No 2, March/April 1988.

[26] F.C. Park and D.J. Pack, "hlotion Control Using the Product-of-Exponentials Kinematic Equations." In 1991 IEEE International Conference on Robotics and Automation. pp. 22042209, Sacramento, April 1991.

[27] Press et al., Numerical Recipes in C. Cambridge University Press, Cambridge, 1988. 
[28] Pro-Matlab User's Guide, 1990.

[29] Rumbelhart and McClelland, Parallel Distributed Processing. MIT Press, Cambridge, 1987.

[30] A.P. Sage and C.C. White. III, Optimum Systems Control.Prentice-Hall, 1977.

[31] F.M.A. Salam and C.S. Yoon, "On the Computational Aspect of the Matrix Exponentials and their use in Robot Kinematics." In IEEE Transactions on Automatic Control. vol. AC-31, No. 4, April 1986.

[32] M. Sekiguchi, T. Sugasaka and S. Nagata, "Control of a Multivariable System by a Neural Network." In 1991 IEEE International Conference on Robotics and Automation. pp. 26442649, Sacramento, April 1991.

[33] R. Sommer, "Control Design for Multivariable Non-linear Time-varying systems" International Journal of Control. vol. 31, pp. 883-891, 1980.

[34] R. Su, G. Meyer and L.R. Hunt, "Robustness in Nonlinear Control" In R.W. Brockett, R.S. Millman and H.J. Sussman,(Eds), Differential Geometric Control Theory. Birkhauser, Boston, vol. 27, pp.268-298, 1983.

[35] T.J. Tarn et al, "Nonlinear Feedback in Robot Arm Control." In Proceedings of the 23rd IEEE Conference on Decision and Control. pp. 736-751, Las Vegas, December 1984.

[36] S. Wolfram, Mathematica. Addison-Wiley, 1990.

[37] W.M. Wonham, Linear Multivariable Control: A Geometric Approach. Springer, Berlin, 1979.

[38] T. Yamaguchi et al, "Intelligent Control of a Flying Vehicle using Fussy Associative Memory System." In 1992 IEEE International Conference on Fuzzy Systems. pp. 1138-1149, San Diego, March 1992.

[39] T. Yamakawa, "Stablization of an Inverted Pendulum by a High-Speed Fuzzy Logic Controller Hardware System." In Fuzzy Sets and Systems. vol. 32, pp. 161-180, 1989.

[40] L.A. Zadeh, "Fuzzy Sets." In Information Control. vol. 8, pp. 338-353, 1965.

[41] S.H. Zak and C.A. MacCarley, "State-feedback control of non-linear systems" International Journal of Control. vol. 43, pp. 1497-1514, 1986. 


\section{APPENDICES}

\section{A Derivation of the observer gain matrix $K$ in section 4.2.2}

As stated in Section 4.3, in order to implement a theoretically well-founded approach to linear control, especially when the full state vector cannot be observed, it is first necessary to determine $\mathrm{K}$, the gain matrix for the observer; this matrix helps the controller to estirnate the state vector from the observed variables. Next, G, the gain matrix for the controller must be calculated, as discussed in Section 4.2.3; the G matrix transforms the estimated state vector into a torque value to be output by the motor.

This appendix addresses the issue of deriving the observer gain matrix K. As stated in Section 4.2.2, $\mathrm{K}$ must be such that the dynamic equation for the estimation error (see Eq. (13)) has certain desirable properties, in the sense that we may want the estimation error to approach zero exponentially without excessive overshoots. Now, whether or not the first-order temporal equation in Eq. (12) possesses an exponential decay and whether or not this exponential decay has a desirable form is determined by the pole locations in the s-plane. ${ }^{8}$ For a linear system governed by Eq. (13), the pole locations are given by the eigenvalues of the matrix $\left(A^{T}-C^{T} K^{T}\right)$. Let the set of eigenvalues of this composite matrix be denoted $\left(\lambda_{1}, \ldots, \mathrm{A}\right.$,). Therefore, at least in principle, all we need do is to find the eigenvalues of $\left(A^{T}-C^{T} K^{T}\right)$ and equate the eigenvalues to wherever we want the poles to be located and then solve for the elements of the $\mathrm{K}$ matrix. Although this approach is straightforward, its major shortcoming is having to solve a coupled set of equations.

A practical alternative is to invoke Lemma stated in Section 4.2.2 which says that every multiinput dynamic system - our error dynamic equation in Eq. (13) is certainly an example of one - can be transformed into an equivalent single-input system. We do that next. The Lemma guarantees that there exists a feedback $F_{0}$ and $c_{0} \in \mathcal{C}^{T}$ such that the original pair $\left(\mathrm{A}^{T}, \mathrm{C}^{T}\right)$ is transformed into the controllable pair $\left(\mathrm{A}^{T}+\mathrm{C}^{T} F_{0}, c_{0}\right)$. The one dimensional column vector $c_{0}$ is found by setting $\mathrm{c}_{0}=\mathrm{C}^{T} \mu$ for some vector $\mu$. The vector $\mu$ can be found through a sequence of tedious steps but is usually found by random number generator that outputs numbers ranging between -1 to 1 . In our case, this $\mu$ vector is a $1 \times 2$ column vector. The above steps can be summarized as setting $\mathrm{u}=\left(F_{0}+\mu \hat{u}\right) e$ in Eq. (13).

'Given the highly interdisciplinary nature of the robotic field, we have endeavored to make this paper readable by even those who are not well conversant with control-theoretic terminology. We would like to state for the benefit of such readers that control systems are frequently analyzed for their stability by analyzing their behavior not directly in the time domain, but in a transform domain - called frequently the s domain. Every function of time has an equivalent representation in the s-plane obtained by multiplying the time function by $e^{-s t}$ and integrating over all time. These representations in the s-plane are characterized by singularities; these are points where the s-plane function goes to infinity. Whether or not a function is decaying in time and how it is decaying depends on the location of the singularities in the s-plane. These points of singularities are referred to as the poles of the function. 
After transforming the system into a single input equivalent system, the error dynamic equation Eq. (13) becomes:

$$
\dot{\mathrm{e}}=\left(\mathrm{A}^{T}+\mathrm{C}^{T} F_{0}\right) \mathrm{e}+\mathrm{c}_{0} \hat{u}
$$

Now we have a single input controllable system. For notational convenience, we will denote $\left(\mathrm{A}^{T}+\mathrm{C}^{T} F_{0}\right)$ as $\mathrm{S}$ and $c_{0}$ as $\mathrm{N}$. Thus, we have the dynamic equation:

$$
\dot{\mathrm{e}}=\mathrm{Se}+\mathrm{N} \hat{u}
$$

Our goal is to work with a dynamic system that has a desirable structure which allows a designer of the system to place poles at desired locations easily. The conversion of multiple input system to its equivalent single input system is the first step to achieve that goal. The next thing we want to do is to find a state transformation when applied to the single input system that yields the pair $(\mathrm{P}, \mathrm{Q})$ with the following controllable canonical form:

$$
\mathrm{P}=\left[\begin{array}{cccccc}
0 & 1 & 0 & 0 & \ldots & 0 \\
0 & 0 & 1 & 0 & \ldots & 0 \\
\vdots & & & & \ddots & \vdots \\
-a_{n} & -a_{n-1} & & & \ldots & -a_{1}
\end{array}\right] \mathbf{Q}=\left[\begin{array}{c}
0 \\
0 \\
\vdots \\
1
\end{array}\right]
$$

we will show next the motivation to transform the pair $(\mathrm{S}, \mathrm{N})$ into the pair $(\mathrm{P}, \mathrm{Q})$. Suppose we have found the transformation and have the system dynamic in the controllable canonical form, and suppose also we have selected a desired set of symmetric $\mathrm{n}$ eigenvalues $\left\{\lambda_{1}, \ldots, \lambda_{n}\right\}$. The dynamic equation, therefore, is described by the following equation

$$
\dot{z}=P z+Q \gamma
$$

where $\gamma$ is the new input to the system. If we set $\gamma=F_{1} z$ then we can compute the corresponding characteristic equation for the matrix $\left(\mathrm{P}+\mathrm{Q} F_{\mathbf{1}}\right)$ as

$$
\begin{aligned}
\Pi_{\mathrm{P}+\mathrm{QF}}(s) & =\left(s-\lambda_{1}\right)\left(s-\lambda_{2}\right) \ldots\left(s-\lambda_{n}\right) \\
& =s^{n}+\hat{a}_{1} s^{n-1}+\ldots+\hat{a}_{n}
\end{aligned}
$$

Note that the characteristic equation of the dynamic equation Eq. (A.4), before any feedback is applied, is

$$
\Pi_{P}(s)=s^{n}+a_{1} s^{n-1}+\ldots+a_{n}
$$

Observe that the coefficients of the characteristic equation are simply the elements of the last row of the matrix P. Thus, if we can replace the coefficients of the original characteristic equation with the coefficients of the desired characteristic equation, we are indeed assigning the new pole locations to be at a set of specified positions. That is, if we set

$$
F_{1}=\left[\begin{array}{lll}
f_{n \text { fn }-1} & \ldots & \mathrm{fl}
\end{array}\right]
$$


the resulting system matrix

$$
P+Q F_{1}=\left[\begin{array}{cccccc}
0 & 1 & 0 & 0 & \ldots & 0 \\
0 & 0 & 1 & 0 & \ldots & 0 \\
\vdots & & & & \ddots & \vdots \\
-a_{n}+f_{n} & -a_{n-1}+f_{n-1} & & & \ldots & -a_{1}+f_{1}
\end{array}\right]
$$

We can now easily solve for the feedback $F_{1}$ by solving the following equations:

$$
\begin{aligned}
& -a_{n}+f_{n}=-\hat{a}_{n} \Rightarrow f_{n}=a_{n}-\hat{a}_{n} \\
& -a_{1}+f_{1}=-\hat{a}_{1} \Rightarrow f_{1}=a_{1}-\hat{a}_{1}
\end{aligned}
$$

We have shown the reason why we want to convert the system into the controllable canonical form above. Now we sho how to find the state transformation that will achieve the desired conversion. We start with Eq. (A.2). Suppose that we have found the linear transformation $\mathrm{Ve}=\mathbf{z}$ that converts the pair $(\mathrm{s}, \mathrm{N})$ into a controllable canonical form:

$$
\dot{z}=\left(\mathrm{VSV}^{-1}\right) z+\mathrm{VN} \hat{u}
$$

where the pair $\left(\mathrm{VSV}^{-1}, \mathrm{VN}\right)$ is in a controllable canonical form shown in matrices $\mathrm{P}$ and $\mathrm{Q}$.

Now we can use the same procedure described above to find the feedback $F_{1}$ such that $\sigma\left(\right.$ VSV $^{-1}+$ $\left.\mathrm{VN} F_{1}\right)=\mathrm{A}$ where $\mathrm{A}$ is a set of desired eigenvalues. When this feedback $\hat{u}=F_{1} \mathbf{z}$ is applied to Eq (A.6), the dynamic equation becomes

$$
\dot{z}=\left(\mathrm{VSV}^{-1}+\mathrm{VN} F_{1}\right) \mathbf{z} .
$$

We can return to the coordinate frame e by applying an inverse transform of $\mathrm{V}$ which yields the dynamic equation:

$$
\mathrm{e}=\left(\mathrm{S}+\mathrm{N} F_{1} \mathrm{~V}\right) \mathrm{e}
$$

Thus, the desired feedback input $\hat{u}$ is $\hat{u}=F_{1} \mathrm{~V}$. We show how to find this transformation matrix V next.

To find this transformation matrix $\mathrm{V}$, we first need to compute the controllability matrix $\mathcal{C}=$ $\left[\mathrm{N} \mathrm{SN} \mathrm{S}{ }^{2} \mathrm{~N} \ldots \mathrm{S}^{n-1} \mathrm{~N}\right]$ where $\mathrm{n}$ denotes the dimension of the matrix $\mathrm{S}$. Then find its inverse matrix $\mathcal{C}^{-1}$ and set its last row to $v$. Now define the $\mathrm{V}$ matrix as

$$
\mathrm{V}=\left[\begin{array}{c}
v \\
v A \\
\vdots \\
v A^{n-1}
\end{array}\right]
$$


We now show that this computed $\mathrm{V}$ is the correct transformation matrix we seek after, as shown in $[S]$. We first prove that from Eq. (A.6), matrix $V \mathbb{N}$ has the form $Q$ in $E q$. (A.5), i.e.,

$$
\mathrm{VN}=\left[\begin{array}{c}
0 \\
0 \\
\vdots \\
1
\end{array}\right]
$$

Recall that $\mathcal{C}^{-1} \mathcal{C}=\mathbf{I}$ where $\mathbf{I}$ is the identity matrix, and

$$
\begin{aligned}
v \mathcal{C} & =v\left[\begin{array}{lllll}
B & A B & A^{2} B & \ldots & A^{n-1} B
\end{array}\right] \\
& =\left[\begin{array}{lllll}
v B & v A B & v A^{2} B & \ldots & v A^{n-1} B
\end{array}\right] \\
& =\left[\begin{array}{lllll}
0 & 0 & \ldots & 0 & 1
\end{array}\right]
\end{aligned}
$$

Thus, $\mathrm{VN}=\left[\begin{array}{llll}0 & 0 & \ldots & 1\end{array}\right]^{T}$ as desired.

We now want to show that $\mathrm{VSV}^{-1}=\hat{S}$, from Eq. (A.6), has the correct controllable canonical form of $\mathrm{P}$ in Eq. (A.3). The equality is true if $\mathrm{VS}=\hat{S} \mathrm{~V}$. Observe that $\mathrm{VS}$ has the following form

$$
\mathrm{VS}=\left[\begin{array}{c}
v \\
v \mathrm{~S} \\
\vdots \\
v \mathrm{~S}^{n-1}
\end{array}\right] \mathrm{S}=\left[\begin{array}{c}
v \mathrm{~S} \\
v \mathrm{~S}^{2} \\
\vdots \\
v \mathrm{~S}^{n}
\end{array}\right]
$$

Eq. (A.7) has to be equal to SV:

$$
\hat{S} \mathrm{~V}=\left[\begin{array}{cccccc}
0 & 1 & 0 & 0 & \ldots & 0 \\
0 & 0 & 1 & 0 & \ldots & 0 \\
\vdots & & & & \ddots & \vdots \\
0 & 0 & 0 & 0 & \ldots & 1 \\
? & ? & ? & ? & \ldots & ?
\end{array}\right]\left[\begin{array}{c}
v \\
v \mathrm{~S} \\
\vdots \\
v \mathrm{~S}^{n-1}
\end{array}\right]
$$

Now the Caley-Hamilton theorem states that

$$
\Pi_{\mathrm{S}}(\mathrm{S})=0=\mathrm{S}^{n}+a_{1} \mathrm{~S}^{n-1}+\ldots+a_{n} I
$$

that is, if we replace the variable $s$ in the characteristic equation with the system matrix $S$, the resulting equation still holds true. This implies that

$$
S^{n}=-a_{n} I-a_{n-1} S-\ldots a_{1} S^{n-1}
$$

and

$$
v S^{\mathrm{n}}=-a_{n} v-a_{n-1} v S-\ldots a_{1} v S^{n-1}
$$


Hence the unknown terms at the bottom of the matrix Eq. (A.8) are exactly equal to $-a_{n},-a_{n-1}, \ldots,-a_{1}$ which we have desired. Thus, the transformation matrix $\mathrm{V}$ when applied to the controllable system Eq. (A.2) yields the controllable canonical form of the pair (P, Q).

To summarize, we have started with a multi-input system Eq. (13) and converted it to a single input equivalent system through input $u=\left(F_{0}+\mu \hat{u}\right) e$. The state transformation $\mathrm{V}$ is applied to the single input dynamic system Eq. (A.4) and the desired pole locations are assigned by the single input feedback $\hat{u}=F_{1} z$. We need to convert this input $\hat{u}$ into the original coordinate frame of e, and we do that by applying the inverse transform of the matrix $\mathrm{V}$ resulting $\hat{u}=F_{1} \mathrm{Ve}$. Thus, the feedback input to the original system Eq. (13) to assign a set of desired pole locations is $u=\left(F_{0}+\mu F_{1} \mathrm{~V}\right)$ e, and the desired $\mathrm{K}$ in Eq. (12) needs to be $\mathrm{K}=-\left[F_{0}+\mu F_{1} \mathrm{~V}\right]^{T}$.

\section{B Derivation of an optimal input for LQ controller}

In Section 4.2.3, we promised to show the reader how an optimal solution can be derived by solving the Ricatti equation Eq. (15). The reader will recall that the system under consideration is described by the dynamic equation Eq. (4) and our optimum solution must minimize the performance criterion in Eq. (14). We believe that the goal we have set out for ourselves in this Appendix is best served if we first consider the scalar version of Eq. (4). The scalar version will help the reader see the logic of the derivation; for the vector case, the notation that one must necessarily use can make it difficult to discern the flow of reasoning. Having presented the main derivational ideas with the help of the scalar case, we will then proceed to the full vector case.

\section{Scalar Case.}

The scalar version of Eq. (4) is

$$
\dot{x}=a(t) x(t)+b(t) \tau(t), x(0)=x_{0} .
$$

and of Eq. (14) that describes the performance measure

$$
\mathrm{V}=\int_{0}^{I_{\text {final }}}\left(x(t)-x^{\text {goal }}(t)\right)^{2} q(t)+\tau^{2}(t) d t
$$

where $\mathrm{x} \in \mathrm{R}$ and where we have assumed for convenience that $T_{\text {initial }}$ is zero. Note that the final value of the state variable, $x\left(T_{\text {final }}\right)$, is free to assume any value that is commensurate with the constraints expressed in the performance measure. Without sacrificing any generality at all, we will now also assume $x^{\text {goal }}(t)=0$; the resulting simplification in the notation will lend us further assistance in making the derivation more transparent. Therefore, we write the following expression for the performance measure:

$$
\mathrm{V}=\int_{0}^{T_{\text {final }}} x(t)^{2} q+\tau^{2}(t) d t
$$


As the reader will recall, the weighting factor $q$ is user-specified. We have not explicitly displayed the weighting factor $r$, because - and it is particularly true for the scalar case - a single weighting factor is sufficient to control the relative importance given to the two contributions to the performance measure.

The immediate goal is to generate the Riccati equation that corresponds to the system being discussed. We do this by first adding terms that do not affect or change the performance measure described in Eq. (B.2). It is clear the following performance measure is same as the one given in Eq. $(B .3)$.

$$
\mathrm{V}=\int_{0}^{T_{\text {final }}}\left[x^{2}(t) q+\tau^{2}(t)+\dot{k}(t) x^{2}(t)+2 k(t) x(t) \dot{x}(t)\right] d t-\left.k(t) x^{2}(t)\right|_{0} ^{T_{\text {final }}}
$$

Note that any $k$ will satisfy Eq. (B.4). Now substitute Eq. (B.l) into Eq. (B.4) to obtain:

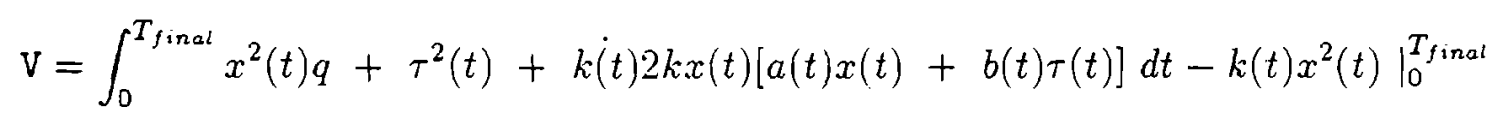

The integrand in the equation can be written in a quadratic form as:

$$
\mathrm{V}=\int_{0}^{T_{\text {final }}}[x(t) \tau(t)]\left[\begin{array}{cc}
q+\dot{k}(t)+2 k(t) a(t) & k(t) b(t) \\
k(t) b(t) & 1
\end{array}\right]\left[\begin{array}{c}
x(t) \\
\tau(t)
\end{array}\right] d t-\left.k(t) x^{2}(t)\right|_{0} ^{T_{\text {final }}}
$$

We would like to rewrite the quadratic terms in the following form:

$$
(\sqrt{\alpha} x(t)+\sqrt{\gamma} u(t))^{2}
$$

If we expand the Eq. (B.6) and equate it to a quadratic, symmetric form, we have

$$
[x(t) \tau(t)]\left[\begin{array}{cc}
\alpha & \sqrt{\alpha \gamma} \\
\sqrt{\alpha \gamma} & \gamma
\end{array}\right]\left[\begin{array}{l}
x(t) \\
\tau(t)
\end{array}\right]=[x(t) \tau(t)]\left[\begin{array}{cc}
\alpha & \beta \\
\beta & \gamma
\end{array}\right]\left[\begin{array}{l}
x(t) \\
\tau(t)
\end{array}\right]
$$

where $\beta=\sqrt{\alpha \gamma}$. Thus, a symmetric quadratic form becomes a perfect square if we have: $\alpha \gamma=\beta^{2}$. This implies that from Eq. (B.5) we need the following equality:

$$
q+\dot{k}(t)+2 k(t) a(t)=k^{2}(t) b^{2}(t) .
$$

This is the sought after Riccati equation for the scalar case. Rearranging the terms gives the final form

$$
\dot{k}(t)=-2 k(t) a(t)+k^{2}(t) b^{2}(t)-q .
$$

Thus, if the function $k$ satisfies the following two conditions,

1. $\dot{k}(t)=-2 k(t) a(t)+k^{2}(t) b^{2}(t)-q$

2. $k\left(T_{\text {final }}\right)=0$ 
the performance index $\mathrm{V}$ becomes

$$
\mathrm{V}=\int_{0}^{T_{f \text { inal }}}[k(t) b(t) x(t)+\tau(t)]^{2} d t-\left.k(t) x^{2}(t)\right|_{0} ^{t_{f}}
$$

and the optimal control input is $\tau(t)=-k(t) b(t) x(t)$. Then, the minimum value of the performance measure is given by $k(0) x^{2}(0)$.

\section{General Case.}

Having made clear, with the help of the scalar case, the main principles that underlie the derivation of the Riccati equation, we are now ready to tackle the general vector case. The dynamic equation is as in Eq. (4), repeated here for convenience

$$
\dot{\mathrm{x}}=A(t) \mathbf{x}(t)+B(t) \tau(t)
$$

where the initial state $\mathbf{x}(0)$ is given and the goal state $\mathbf{x}\left(t_{f}\right)$ is free although we would like it to be $x^{\text {goal }}$. The performance measure is given by Eq. (14) with $Q$ and $R$ as the weighting matrices. Simply for notational convenience, in the derivation to follow we will first assume that $R$ in a identity matrix. Subsequently. we will show how to account for the more general R. Therefore, for our immediate purposes, we write the following expression for the performance measure:

$$
V=\int_{0}^{t_{f}} \mathbf{x}^{T}(t) Q \mathbf{x}(t)+\tau^{T}(t) \tau(t) d t
$$

where we have again assumed, without any loss of generality, that $\mathrm{x}^{\text {goal }}=0$.

In a manner similar to the scalar case, we can reformulate this performance measure as

$$
\mathrm{V}=\int_{0}^{T_{\text {final }}}\left[\mathrm{x}^{T}(t) \mathrm{Q} \mathbf{x}(t)+\tau^{T}(t) \tau(t)+\frac{d}{d t}\left(\mathbf{x}^{T}(t) \mathrm{K}(t) \mathbf{x}(t)\right)\right] d t-\left.\mathrm{x}^{T}(t) \mathrm{K}(t) \mathbf{x}(t)\right|_{0} ^{T_{\text {final }}}
$$

Substituting $\mathrm{Eq}(B .8)$ and writing it in quadratic matrix form yields

$$
\mathrm{V}=\int_{0}^{t_{f}}[\mathbf{x}(t) \tau(t)]\left[\begin{array}{cc}
\mathrm{Q}+\dot{\mathrm{K}}(t)+\mathrm{K}(t) A(t) & \mathrm{K}(t) B(t) \\
B^{T}(t) \mathrm{K}(t) & I
\end{array}\right]\left[\begin{array}{c}
\mathbf{x}(t) \\
\tau(t)
\end{array}\right] d t-\left.\mathrm{x}^{T}(t) \mathrm{K}(t) \mathbf{x}(t)\right|_{0} ^{T_{\text {final }}}
$$

Again as was done for the scalar case, we want the integrand of Eq. (B.9) to have the following form.

$$
\begin{gathered}
\left(B^{T}(t) \mathrm{K}(t) \mathbf{x}(t)+\tau(t)\right)^{T}\left(B^{T}(t) \mathrm{K}(t) \mathbf{x}(t)+\tau(t)\right)= \\
{[\mathbf{x}(t) \tau(t)]\left[\begin{array}{cc}
\mathrm{K}(t) B(t) B^{T}(t) \mathrm{K}(t) & \mathrm{K}(t) B(t) \\
B^{T}(t) \mathrm{K}(t) & I
\end{array}\right]\left[\begin{array}{l}
\mathbf{x}(t) \\
\tau(t)
\end{array}\right]}
\end{gathered}
$$

If we now set $B^{T}(t) \mathrm{K}(t) \mathrm{x}(t)+\tau(t)=0$, we will minimize the performance measure $\mathrm{V}$ since the integral term will simply vanish.

Therefore, similar to the scalar case, we want $\mathrm{K}(t)$ to satisfy the following two conditions: 
1. $\mathrm{Q}+\dot{\mathrm{K}}(t)+\mathrm{K}(t) A(t)+A^{T}(t) \mathrm{K}(t)=\mathrm{K}(t) B(t) B^{T}(t) \mathrm{K}(t)$

2. $K\left(t_{f}\right)=0$

In conclusion, $\tau(t)=-B^{T}(t) \mathrm{K}(t) \mathbf{x}(t)$ gives the optimal control input, and the minimum value of the performance measure is $\mathrm{V}_{\min }=\mathrm{x}^{T}(0) \mathrm{K}(0) \mathrm{x}(0)$.

So far we have ignored the $\mathrm{R}$ weighting factor in the performance measure in Eq. (14). As we said before, this was done for notional clarity in the derivation we have shown so far. We will now explain that the $\mathrm{R}$ factor is taken care of trivially.

Equations (4) and (14) can be recast into the following forms:

$$
\begin{gathered}
\dot{\mathrm{x}}(t)=A(t) \mathbf{x}(t)+B(t)(\sqrt{R})^{-1} \mathrm{v} \\
\mathrm{V}=\int_{0}^{t_{f}} \mathbf{x}^{T}(t) \mathrm{Q}(t) \mathbf{x}(t)+\mathrm{v}^{T}(t) \mathrm{v}(t) d t
\end{gathered}
$$

where we have set

$$
\tau=\sqrt{\mathrm{R}}^{-1} \mathrm{v}
$$

We will now justify our employing the variable $v$ by demonstrating that $\sqrt{R}$ and its inverse do exist such that

$$
\begin{gathered}
\sqrt{R} \sqrt{R}=R \\
\sqrt{R}^{-1} \sqrt{R}=I
\end{gathered}
$$

Since $\mathrm{R}$ is a symmetric and positive definite matrix, it has a set of real eigenvalues. If $\mathrm{R}$ is a diagonal matrix with elements $r_{i, i}, i=1,2, \ldots, \mathrm{n}$ where $\mathrm{n}$ is the dimensionality of the input vector, we can write

$$
\sqrt{R}=\left[\begin{array}{cccc}
r_{11} & 0 & 0 & 0 \\
0 & r_{22} & 0 & 0 \\
0 & 0 & . & . \\
0 & 0 & . & r_{n n}
\end{array}\right]^{\frac{1}{2}}=\left[\begin{array}{cccc}
\sqrt{r_{11}} & 0 & 0 & 0 \\
0 & \sqrt{r_{22}} & 0 & 0 \\
0 & 0 & . & . \\
0 & 0 & \cdot & \sqrt{r_{n n}}
\end{array}\right]
$$

If $R$ is not a diagonal matrix, we can always find a matrix $\Theta$ such that $\Theta^{T} R \Theta=M$ where $M$ is a diagonal matrix and $\Theta^{T} \Theta=\mathbf{I}$. Note that we can find $\sqrt{R}$ by

$$
M^{\frac{1}{2}}=\Theta^{T}(R)^{\frac{1}{2}} \Theta
$$

because

$$
\Theta^{T}(\mathrm{R})^{\frac{1}{2}} \Theta \Theta^{T}(\mathrm{R})^{\frac{1}{2}} \Theta=\Theta^{T} \mathrm{R} \Theta=\mathrm{M}
$$




\section{$C$ Derivation of the transformation for the nonlinear con- troller}

The following derivation has appeared in [41] and is included here for completeness. Given the nonlinear dynamic system $\mathrm{x}=\mathrm{f}(\mathrm{x})+g(\mathrm{x}) \tau$ where $\mathrm{x} \in \mathrm{R}^{\mathrm{n}}, \mathrm{z} \in \mathrm{R}$, we want to find a transformation $\mathbf{z}=\mathrm{T}: \boldsymbol{R}^{\boldsymbol{n}} \rightarrow \boldsymbol{R}^{n}$ such that the dynamic equations in the new coordinate frame have the following form:

$$
\dot{\mathbf{z}}=\left[\begin{array}{c}
z_{2} \\
z_{3} \\
\vdots \\
z_{n} \\
\mathbf{w}\left(z_{1}, z_{2}, \ldots, z_{n}\right)
\end{array}\right]+\left[\begin{array}{c}
0 \\
0 \\
\vdots \\
0 \\
0 \\
1
\end{array}\right] \tau
$$

where $\mathrm{w}$ is, in general, a nonlinear function. Assume that the required transformation is $\mathrm{T}$ and that $\mathrm{T}^{-1}$ exists. Differentiating $\mathrm{z}$ with respect to time gives

$$
\begin{aligned}
\dot{\mathbf{z}} & =\frac{\partial \mathrm{T}}{\partial \mathrm{x}} \frac{\partial \mathrm{x}}{\partial t} \\
& =\frac{\partial \mathrm{T}}{\partial \mathbf{x}}(f(\mathbf{x})+g(\mathbf{x}) \tau) \\
& =\left.\left(\frac{\partial \mathrm{T}}{\partial \mathbf{x}} f(\mathbf{x})\right)\right|_{\mathbf{x}=T^{-1}(z)}+\left.\left(\frac{\partial \mathrm{T}}{\partial \mathbf{x}} g(\mathbf{x})\right)\right|_{\mathbf{x}=T^{-1}(\mathbf{z})} \tau
\end{aligned}
$$

Comparing the above equation with Eq. (C.1) and equating term by term gives,

$$
\left.\frac{\partial \mathrm{T}}{\partial \mathbf{x}} f(\mathbf{x})\right|_{\mathbf{x}=\mathrm{T}^{-1}(\mathbf{z})}=\left[\begin{array}{c}
z_{2} \\
z_{3} \\
\vdots \\
z_{n} \\
\mathbf{w}(\mathbf{z})
\end{array}\right]=\left[\begin{array}{c}
\mathrm{T}_{2} \\
\mathrm{~T}_{3} \\
\vdots \\
\mathrm{T}_{n} \\
\mathrm{w}(\mathbf{z})
\end{array}\right]
$$

and

$$
\left.\frac{\partial \mathrm{T}}{\partial \mathbf{x}} g(\mathbf{x})\right|_{\mathbf{x}=T^{-1}(\mathbf{z})}=\left[\begin{array}{c}
0 \\
0 \\
\vdots \\
0 \\
1
\end{array}\right]
$$

because

$$
\mathbf{z}=\left[\begin{array}{c}
z_{1} \\
z_{2} \\
\vdots \\
z_{n}
\end{array}\right]=\mathrm{T}(\mathbf{x})
$$


In what follows we denote the transpose of the gradient of a real-valued function $n$, $(V n F$, as $\mathrm{d} n$; note that $\mathrm{d} n$ will be a row vector. Using this notation, observe that

$$
\begin{aligned}
\left.\frac{\partial \mathrm{T}}{\partial \mathrm{x}} f(\mathbf{x})\right|_{\mathbf{x}=\mathrm{T}^{-1}(\mathrm{z})} & =\left[\begin{array}{c}
d \mathrm{~T}_{1} \\
d \mathrm{~T}_{2} \\
\vdots \\
d \mathrm{~T}_{n}
\end{array}\right] f \\
& =\left[\begin{array}{c}
<d \mathrm{~T}_{1}, f> \\
<d \mathrm{~T}_{2}, f> \\
\vdots \\
<d \mathrm{~T}_{n}, f>
\end{array}\right] \\
& =\left[\begin{array}{c}
\mathrm{L}_{f} \mathrm{~T}_{1} \\
\mathrm{~L}_{f} \mathrm{~T}_{2} \\
\vdots \\
\mathrm{L}_{f} \mathrm{~T}_{\mathrm{n}}
\end{array}\right]=\left[\begin{array}{c}
\mathrm{T}_{2} \\
\mathrm{~T}_{3} \\
\vdots \\
\mathrm{w}(\mathrm{z})
\end{array}\right]
\end{aligned}
$$

where $<d \mathrm{~T}_{i}, \mathrm{f}>$ denotes the inner product of the vector $d \mathrm{~T}$ and $f$, the latter being a vector function. The last equality above follows from Eq. (C.3). The symbol $L_{f}$ was defined in Section 4.4.1.

The above equation says that if we know $T_{1}$, we can derive the rest of the transformation by

$$
\begin{gathered}
\mathrm{T}_{2}=\mathrm{L}_{f} \mathrm{~T}_{1} \\
\mathrm{~T}_{3}=\mathrm{L}_{f} \mathrm{~T}_{2}=\mathrm{L}_{f} \mathrm{~L}_{f} \mathrm{~T}_{1} \\
\mathrm{~T}_{n}=\mathrm{L}_{\mathbf{j}}^{n-1} \mathrm{~T}_{1}
\end{gathered}
$$

Thus, the transformation matrix becomes

$$
\mathrm{T}=\left[\begin{array}{c}
\mathrm{T}_{1} \\
\mathrm{~L}_{f} \mathrm{~T}_{1} \\
\vdots \\
\mathrm{L}_{f}^{n-1} \mathrm{~T}_{1}
\end{array}\right]
$$

For a linear system, for which the term $f(\mathbf{x})$ in the dynamic equation is replaced by $A x$, the relationship between $z$ and $x$ is given by

$$
\mathrm{z}=\left[\begin{array}{c}
q \\
q \mathrm{~A} \\
\vdots \\
q \mathrm{~A}^{n-1}
\end{array}\right] \mathbf{x}=\left[\begin{array}{c}
\mathrm{T}_{1} \\
\mathrm{~T}_{2} \\
\vdots \\
\mathrm{T}_{n}
\end{array}\right]
$$

where the $q$ is the last row of the inverse matrix of the controllability matrix $\mathrm{C}$. 
Finding the transformation, then, reduces to finding $T_{1}$ and we will address that problem next. It follows from the equality in Eq. (C.4) that

$$
\begin{aligned}
\frac{\partial \mathrm{T}}{\partial \mathrm{x}} \mathrm{g}(\mathrm{x}) & =\left[\begin{array}{c}
d \mathrm{~T}_{1} \\
d \mathrm{~T}_{2} \\
\vdots \\
d \mathrm{~T}_{n}
\end{array}\right] g \\
& =\left[\begin{array}{c}
\mathrm{L}_{g} \mathrm{~T}_{1} \\
\mathrm{~L}_{g} \mathrm{~T}_{2} \\
\vdots \\
\mathrm{L}_{g} \mathrm{~T}_{n}
\end{array}\right]=\left[\begin{array}{c}
0 \\
0 \\
\vdots \\
1
\end{array}\right]
\end{aligned}
$$

Equating the corresponding elements in the above relationship, we get

$$
\mathrm{L}_{3} \mathrm{~T}_{1}=\frac{\partial \mathrm{T}_{1}}{\partial \mathrm{x}}\left(a d^{0} f, g\right)=0
$$

and

$$
\mathrm{L}_{g} \mathrm{~T}_{2}=\mathrm{L}_{g} \mathrm{~L}_{f} \mathrm{~T}_{1}=\frac{\partial \mathrm{T}_{1}}{\partial \mathrm{x}}\left(a d^{1} f, g\right)=0
$$

where we used the result in Eq. (C.6) and where the last equality was obtained by using the Libnietz formula presented in Section 4.4.1. By continuing this process, one gets

$$
\begin{gathered}
\mathrm{L}_{g} \mathrm{~T}_{3}=\frac{\partial \mathrm{T}_{1}}{\partial \mathrm{x}}\left(a d^{2} f, g\right)=0 \\
\mathrm{~L}_{g} \mathrm{~T}_{n}=\frac{\partial \mathrm{T}_{1}}{\partial \mathrm{x}}\left(a d^{n-1} f, g\right)=1
\end{gathered}
$$

Equations from (C.7) to (C.1O) can be combined into the form

$$
\begin{aligned}
& \frac{\partial \mathrm{T}_{1}}{\partial \mathrm{x}}\left[\left(a d^{0} \mathrm{f}, g\right)\left(\mathrm{ad}^{\prime} f, g\right) \ldots\left(\mathrm{ad}^{\mathrm{n}}-^{\prime} \mathrm{f}, g\right)\right]=[0,0, \ldots 0,1]
\end{aligned}
$$

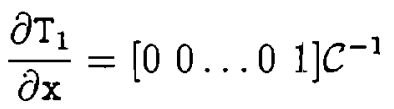

where

$$
\mathcal{C}=\left[\left(\mathrm{ad}^{\prime} \mathrm{f}, g\right)\left(\operatorname{ad}^{1} \mathrm{f}, g\right) \ldots\left(\mathrm{ad}^{\mathrm{n}}-^{\prime} \mathrm{f}, g\right)\right]
$$

is the controllability matrix. So we have the result that $\frac{\partial T_{1}}{\partial x}$ is the last row of $\mathcal{C}^{-1}$. Once this partial differential equation is solved, each component of the transformation $\mathrm{T}$ is determined. 


\section{Proof of Leibniz formula}

We now prove the well known Leibniz formula,

$$
L_{[f, g]} h=L_{g} L_{f} h-L_{f} L_{g} h
$$

We start with the right hand side of Eq. (D.1) and show that it is equal to the left side of Eq. (D.1). We again use the notation $\mathrm{d} n$ as a transpose of a gradient vector of the real-valued function $n$.

$$
\begin{aligned}
L_{g} L_{f} h-L_{f} L_{g} h & =\left(d L_{f} h, g\right)-\left(d L_{g} h, f\right) \\
& =\left(f^{T}\left(\frac{\partial(d h)}{\partial x}\right)+d h\left(\frac{\partial f}{\partial x}\right), g\right)-\left(g^{T}\left(\frac{\partial(d h)}{\partial x}\right)+d h\left(\frac{\partial g}{\partial x}\right), f\right) \\
& =f^{T}\left(\frac{\partial(d h)}{\partial x}\right) g+d h\left(\frac{\partial f}{\partial x}\right) g-g^{T}\left(\frac{\partial(d h)}{\partial x}\right) f-d h\left(\frac{\partial g}{\partial x}\right) f \\
& =d h\left[\left(\frac{\partial f}{\partial x}\right) g-\left(\frac{\partial g}{\partial x}\right) f\right] \\
& =(d h,[f, g]) \\
& =L_{[f, g]} h
\end{aligned}
$$

because $\left(\frac{\partial(d h)}{\partial x}\right)$ is a symmetric matrix when $h$ is twice differentiable.

\section{E Hardware setup}

In this section, we briefly describe the experimental setup. The mechanicad parameters of the pendulum were provided in Section 5, here we will focus on the hardware for the controlling the pendulum. The pendulum is controlled by a transputer, based on the TSOO family by INMOS, mounted on a motherboard that goes into a VME bus. A transputer-based control was chosen to permit us to experiment with more complex systems in the future where we may need to control in real-time multiple motors simultaneously. Basically, a transputer-based system allows parallel and distributed processing, with user-specified synchronizations, at the microprocessor level and that makes it ideal for devices with many degrees of freedom controlled by different actuators. Also, the topology of connections between the different processors can be altered by the user and tailored for each specific task. The connection topology is not an issue for the work reported here, since only one motor is involved. Suffice it to say that the program for controlling the motor, written in $\mathrm{C}$, resides at what is called the root node of the transputer (Fig. 26). Each node in a transputer has four links and one of the links of the root node must be used for the connection with the host machine: in our case a SUN computer. The second link of the root node must be connected to a special transputer node, usually designated T2 in the INMOS family of transputers, whose function is to set up the correct interconnection topology in the two crossbar 


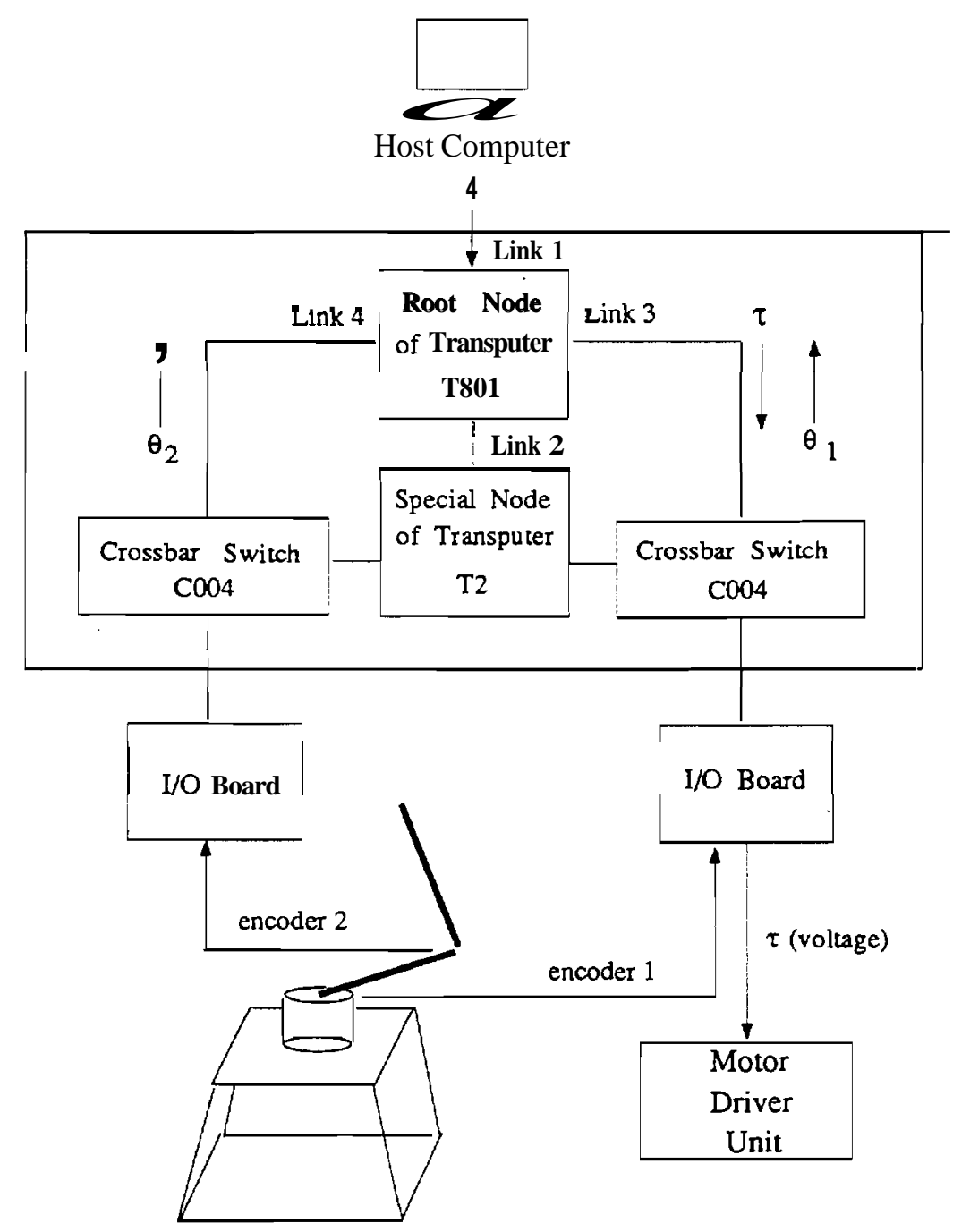

Figure 26: Hardware Configuration 
switches, designated C004 in Fig. 26. [Except for the two links at the root node, all other links for all the other nodes are physically routed into the crossbar switches.]

As shown in Fig. 26, the third and the fourth links of the root node go through the crossbar switches and the I/O boards to the two encoders on the inverted pendulum and to the motor driver. Note especially that the link 3 is used in the full duplex mode, with the outgoing signals corresponding to the motor torque and the incoming signals to the readings of the encoder 1 . The I/O boards consist of a) quadrature decoders which are used to decode the TTL signals generated by the encoders, b) counters for keeping track of encoder counts, c) a digital-to-analog converter for computing an analog voltage value from a discrete digital value calculated by the transputer, d) a clock, etc. The TTL pulses generated by the encoders are basically "tick" marks produced as the optical shaft of the encoder turns. These tick marks are produced on two separate channels, called the quadrature channels; the pulse sequences on the two channels are 180 degrees apart in phase. The function of the decoder is to observe the rise and the fall of the edges of the pulses in the two channels and deduce the direction of rotation of the shaft. Another important function of the decoder is to count the number of pulses to infer the extent of rotation; this the decoder does with the help of the counter. A digital-to-analog converter is also needed on the I/O board for producing the analog voltages for the motor driver.

The control algorithms discussed in this paper produce "nominally" a torque value that the motor is expected to output. The motor is run in what is called a torque mode in which an analog voltage applied to the motor driver results in the motor outputting a proportional value of torque. What is interesting is that the constant of proportionality between what is ostensibly a torque value and the voltage never needs to be made explicit. Consider for example the neural network control algorithm described in this paper. The "torque" output of the neural network is a number between 0 and 1; this output is rescaled to lie between 0 and 255, the lower integer resulting the I/O board outputting an analog voltage of -8 volts and the the upper integer +8 volts. The extreme values of -8 and +8 volts cause the motor to generate the maximum rated torque for the two directions of rotation.

The motor drive unit in Fig. 26 is based on the now well-known H-bridge method [Fig. 27(a)] for controlling the current as well its direction through the windings of a servo motor. The motor windings sit in the horizontal part, the bridge, of the $\mathrm{H}$; the transistor switches in the arms of the $\mathrm{H}$ can be turned on or off to control the direction of the current through the motor windings. The currents in the two paths shown are pulse width modulated so that, except during transitions between the pulses, at any one time the current through the bridge (and therefore through the windings) is in only one direction. By alternately turning on the two current paths shown in the figure and by modulating the widths of the current pulses along these paths (and by using the mechanical inertia of the system), the shaft can be rotated to any desired angle and made to stay there. The actual driver circuitry for the Dynaserve motor we use is somewhat more elaborate; it consists of three $\mathrm{H}$ bridges, as shown in Fig. 27 (b), with each bridge containing separate windings of the motor.

The DYNASERVE motor was selected for its ability to generate a high torque at both slow and high speeds. Also the motor has a large overhung load value which is desirable for the inverted pen- 


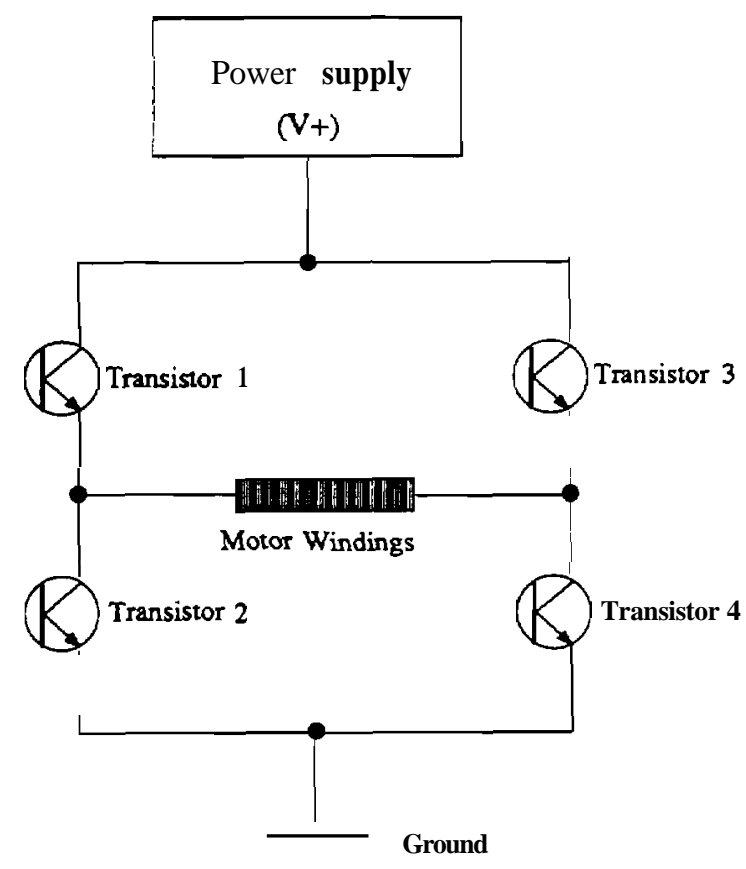

(a.) H-bridge drive

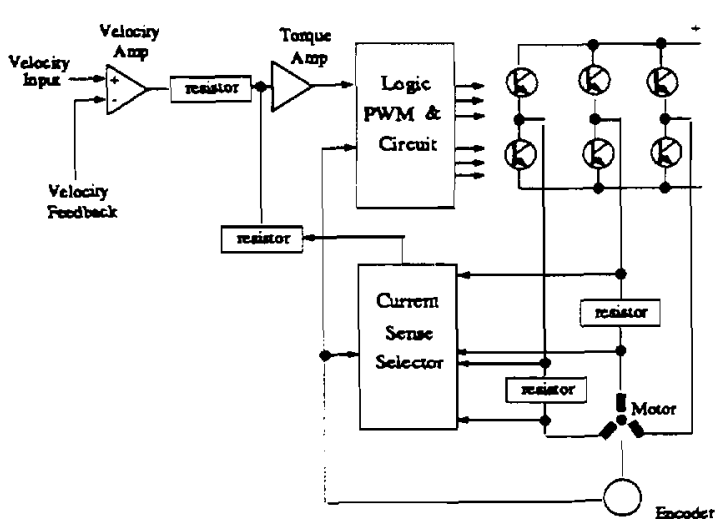

(b) Simplified illustration of the motor drive

Figure 27: Motor Driver

dulum experiments. For encoders we used the TK730 series manufactured by Dynamics Research Corporation. We measure the angular position data directly from the encoders and estimate the velocity data from the position data. 